\title{
Iron-Catalyzed Cross-Coupling of Functionalized Benzylmanganese Halides with Alkenyl lodides, Bromides, and Triflates
}

Alexandre Desaintjean, Sophia Belrhomari, Lidie Rousseau, Guillaume Lefèvre and Paul Knochel

Department of Chemistry, Ludwig-Maximilians-Universität München,

Butenandstr. 5-13, Haus F, 81377 Munich, Germany

paul.knochel@cup.uni-muenchen.de

Chimie ParisTech, PSL University, CNRS, Institute of Chemistry for Life and Health Sciences, CSB2D,

75005 Paris, France

guillaume.lefevre@chimieparistech.psl.eu

\section{Supporting Information}

\section{Table of Contents}

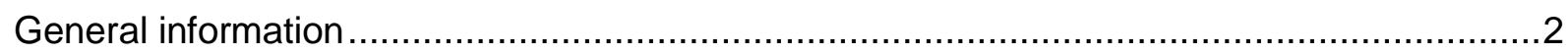

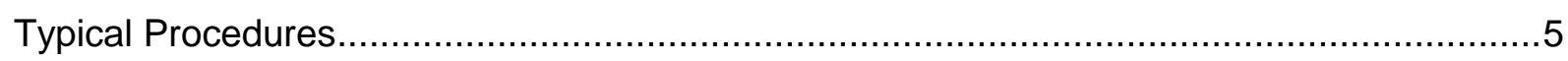

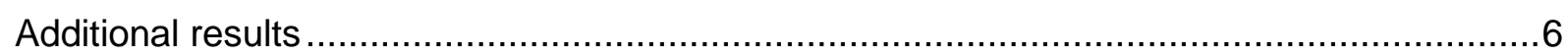

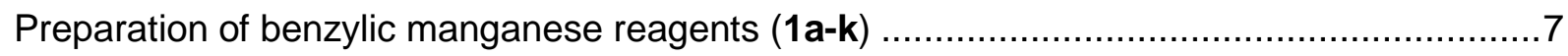

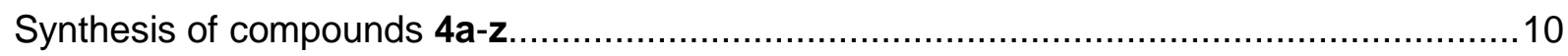

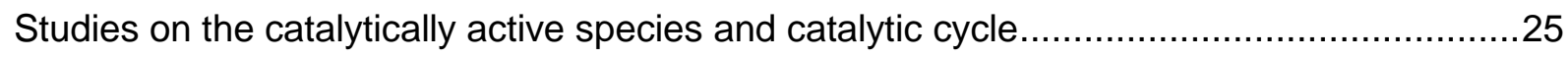

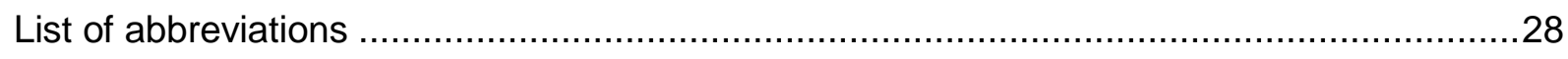

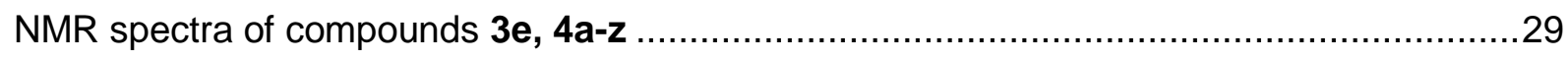




\section{General information}

All reactions, except otherwise noted, were carried out in flame-dried glassware equipped with magnetic stirring under argon atmosphere using standard Schlenk techniques. To transfer solvents or reagents, syringes were used, which were purge three times with argon prior to use. After purification by flash column chromatography, products were concentrated using a rotary evaporator and subsequently dried under high vacuum. Indicated yields are isolated yields of compounds estimated to be $>95 \%$ pure as determined by ${ }^{1} \mathrm{H}-\mathrm{NMR}\left(25^{\circ} \mathrm{C}\right)$ and capillary GC.

To examine the reaction progress of the performed reactions, GC-analysis of quenched hydrolyzed and iodolized reaction aliquots relative to an internal standard was used. For this purpose, small amounts of the reaction mixture were hydrolyzed using a saturated aqueous solution of $\mathrm{NH}_{4} \mathrm{Cl}$, subsequently extracted with $\mathrm{EtOAc}$, dried over $\mathrm{MgSO}_{4}$ and gaschromatographically quantified. To monitor the process of directed metalations and oxidative insertion reactions small amounts of the reaction mixture were iodolyzed. A small quantity of iodine was dissolved in freshly distilled THF $(0.50 \mathrm{~mL})$, charged with the reaction mixture and a solution of $\mathrm{Na}_{2} \mathrm{~S}_{2} \mathrm{O}_{3}$ was added. The mixture was extracted with EtOAc, dried over $\mathrm{MgSO}_{4}$ and was then gaschromatographically measured.

To determine the concentration of the different synthesized metallic reagents iodometric titration was used. For this purpose, a known amount of iodine was charged with freshly distilled THF $(1.00 \mathrm{~mL})$ to give a deep red solution. The metallic reagent was added dropwise at $2^{\circ} \mathrm{C}$ to the iodine solution until the red coloration went to colorless. The concentration of the organometallic reagent could be calculated via the consumed volume of the reaction mixture and the amount of used iodine.

\section{Chromatography}

Thin layer chromatography (TLC) was implemented on alumina plates coated with $\mathrm{SiO}_{2}$ (Merck 60, F-254). To visualize the spots of the different products, UV light was used.

Flash column chromatography was performed using $\mathrm{SiO}_{2}(0.04-0.06 \mathrm{~mm}, 230-400$ mesh) from Merck.

\section{Analytics}

${ }^{1} \mathrm{H}$ NMR, ${ }^{13} \mathrm{C}$ NMR, ${ }^{19} \mathrm{~F}$ NMR and 2D NMR spectra were recorded on VARIAN Mercury 200, BRUKER ARX 300, VARIAN VXR 400 S and BRUKER AMX 600 instruments. Chemical shifts are reported as $\delta$ values in ppm relative to tetramethylsilane. The following abbreviations were used to characterize signal multiplicities: $s$ (singlet), d (doublet), $t$ (triplet), q (quartet), $m$ (multiplet).

Mass spectroscopy: High resolution (HRMS) and low resolution (MS) spectra were recorded on a FINNIGAN MAT 95Q instrument. Electron impact ionization (EI) was conducted with an ionization energy of $70 \mathrm{eV}$. For coupled gas chromatography / mass spectrometry, a HEWLETT-PACKARD HP 6890 /MSD 5973 GC/MS system was used. Molecular fragments are reported starting at a relative intensity of $10 \%$.

Infrared spectra (IR) were recorded from $4500 \mathrm{~cm}^{-1}$ to $650 \mathrm{~cm}^{-1}$ on a PERKIN ELMER Spectrum BX59343 instrument. For detection, a SMITHS DETECTION DuraSampIIR IIDiamond ATR sensor was used. Wavenumbers are reported in $\mathrm{cm}^{-1}$ starting at an absorption of $10 \%$.

Melting points (m.p.) were determined on a BÜCHI B-540 melting point apparatus and are uncorrected. Compounds decomposing upon melting are indicated by (decomp.). 
Gas chromatography was executed with machines of type Agilent Technologies 7890A GC-Systems with 6890 GC inlets, detectors, a GC oven and a column of type HP 5 (Hewlett-Packard, 5\% phenylmethylpolysiloxane; length: $10 \mathrm{~m}$, diameter: $0.25 \mathrm{~mm}$, film thickness: $0.2 \mu \mathrm{m}$ ).

Gas chromatography-Mass spectra were recorded on a networking system called Hewlett-Packard 6890/MSD 5973 GC/MS with a column of type HP 5 (Hewlett-Packard, 5\% phenylmethylpolysiloxane; length: $10 \mathrm{~m}$, diameter: $0.25 \mathrm{~mm}$, film thickness: $0.2 \mu \mathrm{m}$ ).

\section{Chemicals}

All chemicals were purchased from commercial sources and were used without any further purification unless otherwise noted.

\section{Solvents}

THF was continuously refluxed and freshly distilled from benzophenone ketyl under nitrogen. The freshly distilled THF was stored over molecular sieve $(4 \AA)$ under argon. Solvents for cross-coupling reactions such as MTBE and NMP were heated to reflux for $14 \mathrm{~h}$ over $\mathrm{CaH}_{2}$ and distilled. Solvents for column chromatography were distilled prior to use.

\section{Reagents and starting materials}

\section{$\mathrm{MnCl}_{2} \cdot 2 \mathrm{LiCl}(1.00 \mathrm{M}$ in THF):}

A dry and argon-flushed $250 \mathrm{~mL}$ Schlenk-flask, equipped with a magnetic stirring bar and a rubber septum, was charged with $\mathrm{LiCl}\left(8.48 \mathrm{~g}, 200 \mathrm{mmol}, 2.00\right.$ equiv), heated to $450{ }^{\circ} \mathrm{C}$ under high vacuum and after cooling to room temperature under vacuum furthermore vigorously stirred at $160{ }^{\circ} \mathrm{C}$ for $3 \mathrm{~h}$. Subsequently, $\mathrm{MnCl}_{2}$ (12.6 g, $100 \mathrm{mmol}, 1.00$ equiv) was added under argon at room temperature and the reaction mixture as heated to $160^{\circ} \mathrm{C}$ for $3 \mathrm{~h}$ under high vacuum. After cooling to room temperature, the flask as charged with freshly distilled THF $(100 \mathrm{~mL})$ and the mixture was stirred for $48 \mathrm{~h}$ at $25^{\circ} \mathrm{C}$. The resulting $\mathrm{MnCl}_{2} \cdot 2 \mathrm{LiCl}(1.00 \mathrm{M}$ in THF) solution appeared as a light brown liquid.

\section{3-methoxybenzylmagnesium chloride (5):}<smiles>CCc1cccc(OC)c1</smiles>

A dry and argon-flushed Schlenk-tube, equipped with a magnetic stirring bar and a rubber septum, was charged with magnesium turnings (292 $\mathrm{mg}, 12.0 \mathrm{mmol}, 2.40$ equiv), followed by freshly distilled THF (4.00 mL). 3-methoxybenzyl chloride (2a, $783 \mathrm{mg}, 5.00 \mathrm{mmol}, 1.00$ equiv) diluted in THF (10.0 mL) was added to the reaction mixture: $1.00 \mathrm{~mL}$ was added until the solution gets warm then an autopulsed syringe was used (rate: $0.02 \mathrm{~mL} / \mathrm{min}$ ).

lodine $(10.0 \mathrm{mg}, 40.0 \mu \mathrm{mol})$ was added to activate the magnesium turnings.

The reaction mixture was stirred at room temperature until a complete conversion of the starting material was observed. The reaction progress was monitored by GC-analysis of hydrolyzed and iodolyzed aliquots.

When the metalation was completed, the solution of the benzylmagnesium chloride was separated from the magnesium turnings using a syringe and subsequently transferred into another pre-dried and argonflushed Schlenk-tube. To determine the concentration of the magnesium chloride, a titration against iodine in freshly distilled THF was performed. 3-methoxybenzylmagnesium chloride (5) was obtained (0.27 M, 48\%). 


\section{3-methoxybenzylzinc chloride (6):}<smiles>COc1cccc(CC(C)C)c1</smiles>

A dry and argon-flushed Schlenk-tube, equipped with a magnetic stirring bar and a rubber septum, was charged with $\mathrm{LiCl}\left(318 \mathrm{mg}, 7.50 \mathrm{mmol}, 1.25\right.$ equiv) and heated up to ca. $450^{\circ} \mathrm{C}$ for $5 \mathrm{~min}$ under high vacuum using a heat gun. After cooling to room temperature under vigourous stirring, $\mathrm{ZnCl}_{2}(900 \mathrm{mg}$, $6.60 \mathrm{mmol}, 1.10$ equiv) was added under argon, the Schlenk-tube was heated to ca. $320^{\circ} \mathrm{C}$ for $5 \mathrm{~min}$ under vacuum using a heat gun, cooled to room temperature and charged with magnesium turnings (340 mg, $14.4 \mathrm{mmol}, 2.40$ equiv).

Freshly distilled THF (5.00 mL) and 3-methoxybenzyl chloride (2a, $940 \mathrm{mg}, 6.00 \mathrm{mmol}, 1.00$ equiv) were added and the reaction mixture was stirred at room temperature until a complete conversion of the starting material was observed.

The completion of the metalation was monitored by GC-analysis of hydrolyzed and iodolyzed aliquots. When the oxidative insertion was complete, the solution 3-methoxybenzylzinc chloride was separated from the resulting salts via a syringe equipped with a filter and transferred to another pre-dried and argon-flushed Schlenk-tube, before being titrated against iodine.

3-methoxybenzylzinc chloride (6) was obtained (0.39 M, 42\%).

\section{Synthesis of 2,2-diphenylvinyl trifluoromethanesulfonate (3e)}

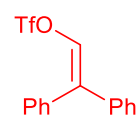

For this reaction, 2,2-diphenylacetaldehyde ( $5.00 \mathrm{~g}, 4.52 \mathrm{~mL}, 25.5 \mathrm{mmol}, 1.00$ equiv), potassium tertbutoxide $(4.05 \mathrm{~g}, 36.1 \mathrm{mmol}, 1.40$ equiv) and THF $(150 \mathrm{~mL})$ were used. The reaction mixture was refluxed for $4 \mathrm{~h}$ and quenched with $\mathrm{N}$-phenyl-bis(trifluoromethanesulfonimide) (10.9 g, $30.6 \mathrm{mmol}, 1.20$ equiv). Purification by flash column chromatography $\left(\mathrm{SiO}_{2}\right.$, isohexane, $\left.\mathrm{R}_{\mathrm{f}}=0.34\right)$ afforded the desired product $3 \mathrm{e}(4.47 \mathrm{~g}, 13.6 \mathrm{mmol}, 54 \%)$ as a yellowish oil.

${ }^{1} \mathrm{H}-\mathrm{NMR}\left(400 \mathrm{MHz}, \mathrm{CDCl}_{3}, \mathrm{ppm}\right) \delta=7.45-7.33(\mathrm{~m}, 6 \mathrm{H}), 7.31-7.22(\mathrm{~m}, 4 \mathrm{H}), 7.05(\mathrm{~s}, 1 \mathrm{H})$.

${ }^{13} \mathrm{C}-\mathrm{NMR}\left(101 \mathrm{MHz}, \mathrm{CDCl}_{3}, \mathrm{ppm}\right) \delta=136.6,134.3(2 \mathrm{C}), 132.0,129.8,129.1,128.9(2 \mathrm{C}), 128.6(2 \mathrm{C})$, $118.6(q, J=321.2 \mathrm{~Hz}$ ).

${ }^{19} \mathrm{~F}-\mathrm{NMR}\left(377 \mathrm{MHz}, \mathrm{CDCl}_{3}, \mathrm{ppm}\right) \delta=-73.7$.

IR $\left(\mathbf{A T R}, \mathbf{c m}^{-1}\right) \tilde{v}=1446,1422,1244,1205,1165,1137,1035,1014,947,914,828,807,775,759,694$.

MS (EI, 70 eV, \%) m/z = 195 (60), 167 (100), 165 (79), 152 (39).

HRMS (El, $70 \mathrm{eV}$ ) m/z: calc. for $\mathrm{C}_{15} \mathrm{H}_{11} \mathrm{~F}_{3} \mathrm{O}_{3} \mathrm{~S}$ : 328.0381 ; found 328.0373 . 


\section{Typical Reaction Procedures}

\section{Typical Procedure for the Preparation of Benzylic Manganese Chlorides 1a-k (TP 1)}

A dry and argon-flushed Schlenk-tube, equipped with a magnetic stirring bar and a rubber septum, was charged with magnesium turnings ( $583 \mathrm{mg}, 24.0 \mathrm{mmol}, 2.40$ equiv), followed by freshly distilled THF $(5.00 \mathrm{~mL})$ or MTBE $(6.25 \mathrm{~mL})$ and a solution of $\mathrm{MnCl}_{2} \cdot 2 \mathrm{LiCl}(12.5 \mathrm{~mL}, 12.5 \mathrm{mmol}, 1.20$ equiv, $1.00 \mathrm{M} \mathrm{in}$ THF). The solution was then cooled to $-5^{\circ} \mathrm{C}$. Subsequently, the corresponding benzyl chloride $(\mathbf{2 a - k}$, $10.0 \mathrm{mmol}, 1.00$ equiv) was added drop by drop and the reaction mixture was stirred at $-5{ }^{\circ} \mathrm{C}$ until a complete conversion of the starting material was observed. The reaction progress was monitored by GC-analysis of hydrolyzed and iodolyzed aliquots.

When the metalation was completed, the black solutions of the benzylmanganese chlorides 1a-k were separated from the magnesium turnings using a syringe and subsequently transferred into another predried and argon-flushed Schlenk-tube, which was cooled to $-5^{\circ} \mathrm{C}$. To determine the concentration of the manganese chlorides 1a-k a titration against iodine in freshly distilled THF was performed.

\section{Typical Procedure for the Fe-catalyzed Cross-Coupling Reactions of Benzylic metallic species 1a-k, 5, 6 with different Elecrophiles 3a-n (TP 2)}

A pre-dried and argon-flushed Schlenk-tube equipped with a magnetic stirring bar and a rubber septum was charged with $\mathrm{FeCl}_{2}(4.20 \mathrm{mg}, 33.0 \mu \mathrm{mol}, 10 \mathrm{~mol} \%)$, the corresponding electrophile (3a-n, $333 \mu \mathrm{mol}$, 1.00 equiv), tetradecane as internal standard $(20 \mu \mathrm{L})$ and freshly distilled THF $(0.30 \mathrm{~mL})$ as solvent. The reaction mixture was cooled to $0^{\circ} \mathrm{C}$ and the benzylic metallic species solution (1a-k, 5, 6, 1.20 equiv) was added dropwise. After the addition was complete, the reaction mixture was allowed to warm to 25 ${ }^{\circ} \mathrm{C}$ and the completion of the cross-coupling reaction was monitored by GC-analysis of hydrolyzed aliquots. Thereupon, a saturated aqueous solution of $\mathrm{NH}_{4} \mathrm{Cl}$ was added and the aqueous layer was extracted with EtOAc $(3 \times 100 \mathrm{~mL})$. The combined organic layers were dried over $\mathrm{MgSO}_{4}$, filtered and concentrated under reduced pressure. Purification of the crude products by flash column chromatography afforded the desired cross-coupling reaction products (4a-z). 


\section{Additional results}

Screening of other benzylic metallic species
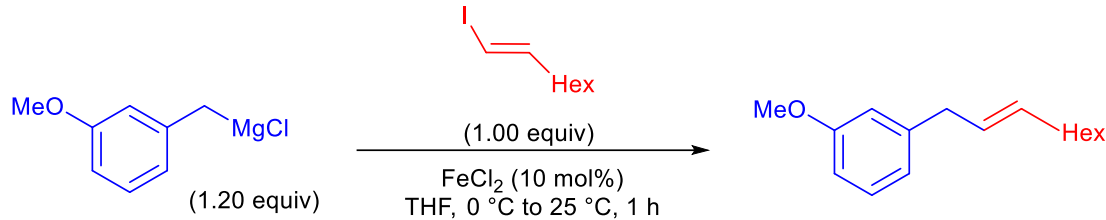

$68 \%$

without catalyst: $47 \%$
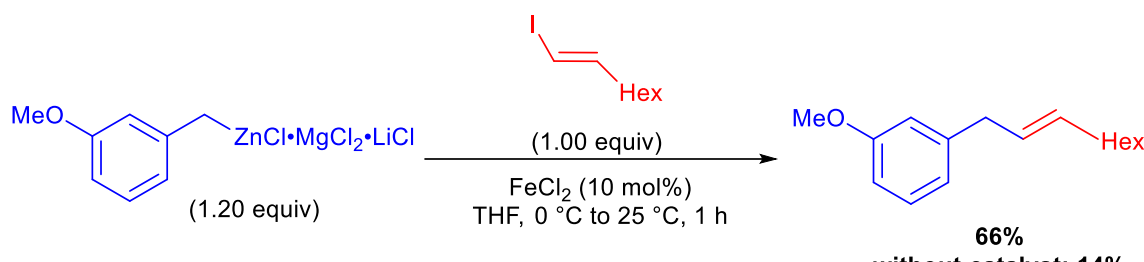

No effect if $\mathrm{RZnCl}$ is more concentrated and/or in excess ( 1.5 equiv)

\section{Screening of NMP as cosolvent and catalyst loading}

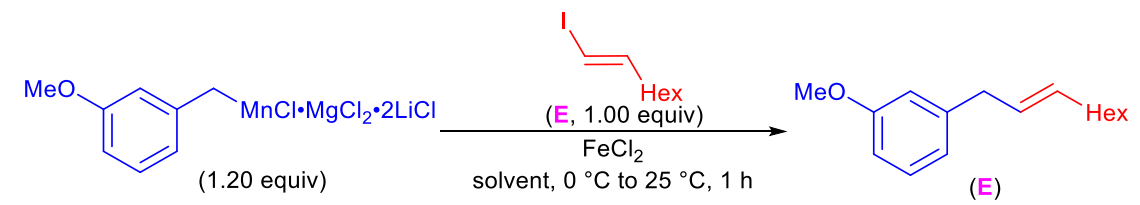

Experiments with $10 \mathrm{~mol} \% \mathrm{FeCl}_{2}$ :

\begin{tabular}{|c|c|}
\hline THF:Cosolvent & $\begin{array}{c}\text { Yield } \\
(\%)\end{array}$ \\
\hline THF:NMP = 1:1 & 97 \\
\hline THF:NMP = 3:1 & 93 \\
\hline THF only & 97 \\
\hline
\end{tabular}

Experiments conducted in THF:

\begin{tabular}{|c|c|}
\hline$\left[\mathrm{FeCl}_{2}\right]$ & $\begin{array}{c}\text { Yield } \\
(\%)\end{array}$ \\
\hline $10 \mathrm{~mol} \%$ & 98 \\
\hline $5.0 \mathrm{~mol} \%$ & 73 \\
\hline $2.5 \mathrm{~mol} \%$ & 79 \\
\hline $1.0 \mathrm{~mol} \%$ & 79 \\
\hline
\end{tabular}




\section{Preparation of benzylic manganese reagents (1a-k)}

\section{Preparation of 3-Methoxybenzylmanganese chloride (1a)}<smiles>COc1cccc(C[Al]Cl)c1</smiles>

Based on TP1, magnesium turnings ( $583 \mathrm{mg}, 24.0 \mathrm{mmol}, 2.40$ equiv), THF (5.00 mL) and a solution of $\mathrm{MnCl}_{2} \cdot 2 \mathrm{LiCl}$ (12.5 mL, $12.5 \mathrm{mmol}, 1.25$ equiv, $1.00 \mathrm{M}$ in THF) were used. Subsequently, 3methoxybenzyl chloride (2a, $1.57 \mathrm{~g}, 1.45 \mathrm{~mL}, 10.0 \mathrm{mmol}, 1.00$ equiv) was added dropwise at $-5^{\circ} \mathrm{C}$ and the reaction mixture was stirred for $1 \mathrm{~h}$ at the given temperature. The concentration of 3methoxybenzylmanganese chloride (1a) was determined against iodine in THF (0.39 M, 78\%).

\section{Preparation of 4-(Methylthio)benzylmanganese chloride (1b)}<smiles>COc1ccc(CCl)cc1</smiles>

Based on TP1, magnesium turnings ( $350 \mathrm{mg}, 14.4 \mathrm{mmol}, 2.40$ equiv), THF ( $3.00 \mathrm{~mL}$ ) and a solution of $\mathrm{MnCl}_{2} \cdot 2 \mathrm{LiCl}(7.50 \mathrm{~mL}, 7.50 \mathrm{mmol}, 1.25$ equiv, $1.00 \mathrm{M}$ in THF) were used. Subsequently, 4(methylthio)benzyl chloride (2b, $1.04 \mathrm{~g}, 0.91 \mathrm{~mL}, 6.00 \mathrm{mmol}, 1.00$ equiv) was added dropwise at $-5{ }^{\circ} \mathrm{C}$ and the reaction mixture was stirred for $1 \mathrm{~h}$ at the given temperature. The concentration of 4(methylthio)benzylmanganese chloride (1b) was determined against iodine in THF (0.36 M, 69\%).

\section{Preparation of benzylmanganese chloride (1c)}<smiles>ClCc1ccccc1</smiles>

Based on TP1, magnesium turnings ( $583 \mathrm{mg}, 24.0 \mathrm{mmol}, 2.40$ equiv), THF (5.00 mL) and a solution of $\mathrm{MnCl}_{2} \cdot 2 \mathrm{LiCl}(12.5 \mathrm{~mL}, 12.5 \mathrm{mmol}, 1.25$ equiv, $1.00 \mathrm{M}$ in THF) were used. Subsequently, benzylchloride (2c, $1.27 \mathrm{~g}, 1.15 \mathrm{~mL}, 10.0 \mathrm{mmol}, 1.00$ equiv) was added dropwise at $-5^{\circ} \mathrm{C}$ and the reaction mixture was stirred for $0.5 \mathrm{~h}$ at the given temperature. The concentration of benzylmanganese chloride (1c) was determined against iodine in THF $(0.34 \mathrm{M}, 65 \%)$.

\section{Preparation of 4-Tertbutylbenzylmanganese chloride (1d)}<smiles>CN(Cl)Cc1ccc(C(C)(C)C)cc1</smiles>

Based on TP1, magnesium turnings (180 $\mathrm{mg}, 7.20 \mathrm{mmol}, 2.40$ equiv), THF (3.00 mL) and a solution of $\mathrm{MnCl}_{2} \cdot 2 \mathrm{LiCl}(3.80 \mathrm{~mL}, 3.80 \mathrm{mmol}, 1.25$ equiv, $1.00 \mathrm{M}$ in THF) were used. Subsequently, 1 -(tert-butyl)4-(chloromethyl)benzene $\left(\mathbf{2 d}, 550 \mathrm{mg}, 0.58 \mathrm{~mL}, 3.00 \mathrm{mmol}, 1.00\right.$ equiv) was added dropwise at $-5{ }^{\circ} \mathrm{C}$ and the reaction mixture was stirred for $1 \mathrm{~h}$ at the given temperature. The concentration of 4-(tertbutyl)benzylmanganese chloride (1d) was determined against iodine in THF (0.37 M, 84\%). 


\section{Preparation of 4-Isopropylbenzylmanganese chloride (1e)}<smiles>CCCc1ccc(CCl)cc1</smiles>

Based on TP1, magnesium turnings ( $350 \mathrm{mg}, 14.4 \mathrm{mmol}, 2.40$ equiv), THF ( $3.00 \mathrm{~mL}$ ) and a solution of $\mathrm{MnCl}_{2} \cdot 2 \mathrm{LiCl}(7.50 \mathrm{~mL}, 7.50 \mathrm{mmol}, 1.25$ equiv, $1.00 \mathrm{M}$ in THF) were used. Subsequently, 4isopropylbenzyl chloride $\left(2 \mathrm{e}, 1.01 \mathrm{~g}, 0.66 \mathrm{~mL}, 6.00 \mathrm{mmol}, 1.00\right.$ equiv) was added dropwise at $-5^{\circ} \mathrm{C}$ and the reaction mixture was stirred for $1.5 \mathrm{~h}$ at the given temperature. The concentration of 4isopropylbenzylmanganese chloride (1e) was determined against iodine in THF $(0.37 \mathrm{M}, 68 \%)$.

\section{Preparation of 2-Fluorobenzylmanganese chloride (1f)}<smiles>Fc1ccccc1CCl</smiles>

Based on TP1, magnesium turnings (175 mg, $7.20 \mathrm{mmol}, 2.40$ equiv), THF (1.50 mL) and a solution of $\mathrm{MnCl}_{2} \cdot 2 \mathrm{LiCl}(3.80 \mathrm{~mL}, 3.80 \mathrm{mmol}, 1.25$ equiv, $1.00 \mathrm{M}$ in THF) were used. Subsequently, 2-fluorobenzyl chloride (2f, $434 \mathrm{mg}, 0.36 \mathrm{~mL}, 3.00 \mathrm{mmol}, 1.00$ equiv) was added dropwise at $-5{ }^{\circ} \mathrm{C}$ and the reaction mixture was stirred for $1.5 \mathrm{~h}$ at the given temperature. The concentration of 2-fluorobenzylmanganese chloride (1f) was determined against iodine in THF $(0.40 \mathrm{M}, 67 \%)$.

\section{Preparation of 3-Fluorobenzylmanganese chloride (1g)}<smiles>Fc1cccc(CCl)c1</smiles>

Based on TP1, magnesium turnings (175 mg, $7.20 \mathrm{mmol}, 2.40$ equiv), THF (1.50 mL) and a solution of $\mathrm{MnCl}_{2} \cdot 2 \mathrm{LiCl}$ ( $3.80 \mathrm{~mL}, 3.80 \mathrm{mmol}, 1.25$ equiv, $1.00 \mathrm{M}$ in THF) were used. Subsequently, 3-fluorobenzyl chloride (2g, $434 \mathrm{mg}, 0.36 \mathrm{~mL}, 3.00 \mathrm{mmol}, 1.00$ equiv) was added dropwise at $-5^{\circ} \mathrm{C}$ and the reaction mixture was stirred for $0.5 \mathrm{~h}$ at the given temperature. The concentration of 3-fluorobenzylmanganese chloride (1) was determined against iodine in THF $(0.30 \mathrm{M}, 68 \%)$.

\section{Preparation of 3-(trifluoromethyl)benzylmanganese chloride (1h)}<smiles>CCc1cccc(C(F)(F)F)c1</smiles>

Based on TP1, magnesium turnings (175 mg, $7.20 \mathrm{mmol}, 2.40$ equiv), MTBE (1.90 mL) and a solution of $\mathrm{MnCl}_{2} \cdot 2 \mathrm{LiCl}(3.80 \mathrm{~mL}, 3.80 \mathrm{mmol}, 1.25$ equiv, $1.00 \mathrm{M}$ in THF) were used. Subsequently, 3(trifluoromethyl)benzyl chloride $(\mathbf{2 h}, 584 \mathrm{mg}, 0.47 \mathrm{~mL}, 3.00 \mathrm{mmol}, 1.00$ equiv) was added dropwise at $5{ }^{\circ} \mathrm{C}$ and the reaction mixture was stirred for $1 \mathrm{~h}$ at the given temperature. The concentration of 3(trifluoromethyl)benzylmanganese chloride (1h) was determined against iodine in THF $(0.31 \mathrm{M}, 58 \%)$. 


\section{Preparation of 2-Chlorobenzylmanganese chloride (1i)}<smiles>ClCc1ccccc1Cl</smiles>

Based on TP1, magnesium turnings (583 mg, $24.0 \mathrm{mmol}, 2.40$ equiv), MTBE (6.25 mL) and a solution of $\mathrm{MnCl}_{2} \cdot 2 \mathrm{LiCl}(12.5 \mathrm{~mL}, 12.5 \mathrm{mmol}, 1.25$ equiv, $1.00 \mathrm{M}$ in THF) were used. Subsequently, 2chlorobenzyl chloride $\left(2 \mathrm{i}, 1.61 \mathrm{~g}, 1.16 \mathrm{~mL}, 10.0 \mathrm{mmol}, 1.00\right.$ equiv) was added dropwise at $-5^{\circ} \mathrm{C}$ and the reaction mixture was stirred for $1 \mathrm{~h}$ at the given temperature. The concentration of 2 chlorobenzylmanganese chloride (1i) was determined against iodine in THF (0.22 M, 44\%).

\section{Preparation of 3-Chlorobenzylmanganese chloride (1j)}<smiles>ClNCc1cccc(Cl)c1</smiles>

Based on TP1, magnesium turnings ( $350 \mathrm{mg}, 14.4 \mathrm{mmol}, 2.40$ equiv), THF (3.00 mL) and a solution of $\mathrm{MnCl}_{2} \cdot 2 \mathrm{LiCl}(7.50 \mathrm{~mL}, 7.50 \mathrm{mmol}, 1.25$ equiv, $1.00 \mathrm{M}$ in THF) were used. Subsequently, 3-chlorobenzyl chloride (2j, $996 \mathrm{mg}, 0.78 \mathrm{~mL}, 6.00 \mathrm{mmol}, 1.00$ equiv) was added dropwise at $-5^{\circ} \mathrm{C}$ and the reaction mixture was stirred for $1.5 \mathrm{~h}$ at the given temperature. The concentration of 3-chlorobenzylmanganese chloride (1j) was determined against iodine in THF (0.47 M, 86\%).

\section{Preparation of 4-Bromobenzylmanganese chloride (1k)}<smiles>ClCc1ccc(Br)cc1</smiles>

Based on TP1, magnesium turnings (180 mg, $7.20 \mathrm{mmol}, 2.40$ equiv), THF ( $3.00 \mathrm{~mL}$ ) and a solution of $\mathrm{MnCl}_{2} \cdot 2 \mathrm{LiCl}(3.80 \mathrm{~mL}, 3.80 \mathrm{mmol}, 1.25$ equiv, $1.00 \mathrm{M}$ in THF) were used. Subsequently, 1-bromo-4(chloromethyl)benzene $\left(\mathbf{2 k}, 620 \mathrm{mg}, 3.00 \mathrm{mmol}, 1.00\right.$ equiv) was added dropwise at $-5^{\circ} \mathrm{C}$ and the reaction mixture was stirred for $2 \mathrm{~h}$ at the given temperature. The concentration of 4-bromobenzylmanganese chloride (1k) was determined against iodine in THF $(0.22 \mathrm{M}, 50 \%)$. 


\section{Synthesis of compounds $4 a-z$}

\section{Synthesis of (E)-1-methoxy-3-(non-2-en-1-yl)benzene (4a)}<smiles>COc1cccc(CC=CC(=O)c2ccccc2)c1</smiles>

According to TP2, for this reaction $\mathrm{FeCl}_{2}(4.20 \mathrm{mg}, 33.0 \mu \mathrm{mol}, 10 \mathrm{~mol} \%),(E)$-1-iodooctene (3a, $79.0 \mathrm{mg}$, $333 \mu \mathrm{mol}, 1.00$ equiv) and freshly distilled THF $(0.30 \mathrm{~mL})$ were used. The reaction mixture was cooled to $0{ }^{\circ} \mathrm{C}$ and 3-Methoxybenzylmanganese chloride $(\mathbf{1 a}, 1.03 \mathrm{~mL}, 400 \mu \mathrm{mol}, 1.20$ equiv, $0.39 \mathrm{M})$ was added dropwise before the mixture was allowed to warm to $25^{\circ} \mathrm{C}$ for $1 \mathrm{~h}$. Purification by flash column chromatography $\left(\mathrm{SiO}_{2}\right.$, isohexane, $\left.\mathrm{R}_{\mathrm{f}}=0.41\right)$ afforded the desired cross-coupling reaction product $4 \mathrm{a}$ (75.0 mg, $323 \mu \mathrm{mol}, 97 \%$ ) as a colorless oil.

${ }^{1} \mathrm{H}-\mathrm{NMR}\left(400 \mathrm{MHz}, \mathrm{CDCl}_{3}, \mathrm{ppm}\right) \delta=7.24-7.18(\mathrm{~m}, 1 \mathrm{H}), 6.81-6.77(\mathrm{~m}, 1 \mathrm{H}), 6.76-6.73(\mathrm{~m}, 2 \mathrm{H}), 5.61$ $-5.47(\mathrm{~m}, 2 \mathrm{H}), 3.80(\mathrm{~s}, 3 \mathrm{H}), 3.31(\mathrm{~d}, J=5.1 \mathrm{~Hz}, 2 \mathrm{H}), 2.08-1.98(\mathrm{~m}, 2 \mathrm{H}), 1.42-1.33(\mathrm{~m}, 2 \mathrm{H}), 1.33-$ $1.23(\mathrm{~m}, 6 \mathrm{H}), 0.93-0.83(\mathrm{~m}, 3 \mathrm{H})$.

${ }^{13} \mathrm{C}-\mathrm{NMR}\left(101 \mathrm{MHz}, \mathrm{CDCl}_{3}, \mathrm{ppm}\right) \delta=159.7,142.9,132.5,129.4,128.6,121.0,114.3,111.3,55.3,39.2$, $32.7,31.9,29.6,29.0,22.8,14.3$.

IR $\left(\right.$ ATR, $\left.\mathbf{~ c m}^{-1}\right) \tilde{v}=2955,2924,2854,1601,1585,1488,1465,1454,1435,1257,1148,1046,966,874$, $771,726,693$.

MS (El, $70 \mathrm{eV}, \%) \mathrm{m} / \mathrm{z}=232$ (80), 175 (11), 161 (31), 150 (10), 147 (87), 136 (16), 134 (100), 128 (10), 122 (99), 117 (17), 91 (48).

HRMS (EI, $70 \mathrm{eV}$ ) m/z: calc. for $\mathrm{C}_{16} \mathrm{H}_{24} \mathrm{O}: 232.1827$; found 232.1821 .

Synthesis of (3-(3-methoxyphenyl)prop-1-en-1-yl)trimethylsilane (4b)<smiles>CNC=CCc1cccc(OC)c1</smiles>

In relation to TP2, for this reaction $\mathrm{FeCl}_{2}(13.0 \mathrm{mg}, 100 \mu \mathrm{mol}, 10 \mathrm{~mol} \%)$, 2-bromovinyltrimethylsilane (3b, $Z / E=10: 90,179 \mathrm{mg}, 0.15 \mathrm{~mL}, 1.00 \mathrm{mmol}, 1.00$ equiv) and freshly distilled THF (1.00 mL) were used. The reaction mixture was cooled to $0{ }^{\circ} \mathrm{C}$ and 3-methoxybenzylmanganese chloride $(\mathbf{1 a}, 3.08 \mathrm{~mL}$, $1.20 \mathrm{mmol}, 1.20$ equiv, $0.39 \mathrm{M}$ ) was added dropwise before the mixture was allowed to warm to $25^{\circ} \mathrm{C}$ for $12 \mathrm{~h}$. Purification by flash column chromatography $\left(\mathrm{SiO}_{2}\right.$, isohexane:ethyl acetate $\left.=99: 1, \mathrm{R}_{\mathrm{f}}=0.54\right)$ afforded the desired cross-coupling reaction product $\mathbf{4 b}(202 \mathrm{mg}, Z / E=6: 94,920 \mu \mathrm{mol}, 92 \%)$ as a colorless oil.

${ }^{1} \mathrm{H}-\mathrm{NMR}\left(400 \mathrm{MHz}, \mathrm{CDCl}_{3}, \mathrm{ppm}\right) \delta=7.26(\mathrm{dd}, J=14.1,6.3 \mathrm{~Hz}, 1 \mathrm{H}), 7.02-6.66(\mathrm{~m}, 3 \mathrm{H}), 6.45$ (dt, $J=$ $13.8,7.4 \mathrm{~Hz}, 0.06 \mathrm{H}), 6.18$ (dt, $J=18.8,6.4 \mathrm{~Hz}, 0.93 \mathrm{H}), 5.75(\mathrm{dt}, J=18.4,1.5 \mathrm{~Hz}, 0.93 \mathrm{H}), 5.73(\mathrm{t}, J=$ $1.5 \mathrm{~Hz}, 0.06 \mathrm{H}),, 3.83(\mathrm{~s}, 3 \mathrm{H}), 3.46(\mathrm{dd}, J=6.2,1.0 \mathrm{~Hz}, 2 \mathrm{H}), 0.18-0.02(\mathrm{~m}, 9 \mathrm{H})$.

${ }^{13} \mathrm{C}-\mathrm{NMR}\left(101 \mathrm{MHz}, \mathrm{CDCl}_{3}, \mathrm{ppm}\right) \delta=159.8,146.7,145.0,142.1,141.8,131.8,130.4,129.6,129.5$, 121.3, 121.0, 114.6, 114.3, 111.6, 111.5, 55.3, 43.4, 39.7, 38, 0.5,-1.1. 
IR $\left(\mathbf{A T R}, \mathbf{c m}^{-1}\right) \tilde{v}=2954,2898,2834,1600,1584,1490,1466,1454,1436,1316,1286,1258,1246$, 1162, 1150, 1052, 1046, 990, 938, 860, 834, 766, 748, 692.

MS (EI, 70 eV, \%) m/z = 220 (28), 205 (100), 193 (12), 190 (60), 175 (47), 146 (71), 121 (15), 115 (23), 91 (13), 75 (14), 73 (58), 59 (18).

HRMS (El, $70 \mathrm{eV}$ ) m/z: calc. for $\mathrm{C}_{13} \mathrm{H}_{20} \mathrm{OSi}$ : 220.1283; found 220.1277 .

\section{Synthesis of trimethyl(3-(4-(methylthio)phenyl)prop-1-en-2-yl)silane (4c)}

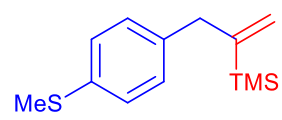

According to TP2, for this reaction $\mathrm{FeCl}_{2}(4.20 \mathrm{mg}, 33.0 \mu \mathrm{mol}, 10 \mathrm{~mol} \%)$, (1-bromovinyl)trimethylsilane (3c, $60.0 \mathrm{mg}, 333 \mu \mathrm{mol}, 1.00$ equiv) and freshly distilled THF $(0.3 \mathrm{~mL})$ were used. The reaction mixture was cooled to $0{ }^{\circ} \mathrm{C}$ and 4-(Methylthio)benzylmanganese chloride (1b, $1.11 \mathrm{~mL}, 400 \mu \mathrm{mol}, 1.20$ equiv, $0.36 \mathrm{M}$ ) was added dropwise before the mixture was allowed to warm to $25^{\circ} \mathrm{C}$ for $12 \mathrm{~h}$. Purification by flash column chromatography $\left(\mathrm{SiO}_{2}\right.$, isohexane:ethyl acetate $\left.=98: 2, \mathrm{R}_{\mathrm{f}}=0.83\right)$ afforded the desired cross-coupling reaction product $\mathbf{4 c}(73.0 \mathrm{mg}, 308 \mu \mathrm{mol}, 92 \%)$ as a yellowish oil.

${ }^{1} \mathrm{H}-\mathrm{NMR}\left(400 \mathrm{MHz}, \mathrm{CDCl}_{3}, \mathrm{ppm}\right) \delta=7.19(\mathrm{~d}, J=8.3 \mathrm{~Hz}, 2 \mathrm{H}), 7.10-7.05(\mathrm{~m}, 2 \mathrm{H}), 5.49-5.40(\mathrm{~m}, 2 \mathrm{H})$, $3.42(\mathrm{~d}, J=1.3 \mathrm{~Hz}, 2 \mathrm{H}), 2.47(\mathrm{~s}, 3 \mathrm{H}), 0.00(\mathrm{~s}, 9 \mathrm{H})$.

${ }^{13} \mathrm{C}-\mathrm{NMR}\left(101 \mathrm{MHz}, \mathrm{CDCl}_{3}, \mathrm{ppm}\right) \delta=151.4,137.4,135.5,129.9,126.9,126.0,42.3,16.4,-1.3$.

IR $\left(\right.$ ATR, $\left.\mathbf{c m}^{-1}\right) \tilde{v}=2955,2921,1492,1436,1404,1258,1247,1093,922,836,800,759,691$.

MS (El, 70 eV, \%) m/z = 236 (83), 221 (79), 206 (11), 191 (15), 181 (11), 137 (37), 115 (21), 73 (100).

HRMS (El, $70 \mathrm{eV}$ ) m/z: calc. for $\mathrm{C}_{13} \mathrm{H}_{20} \mathrm{SSi}$ : 236.1055; found 236.1047 .

\section{Synthesis of (E)-non-2-en-1-ylbenzene (4d)}

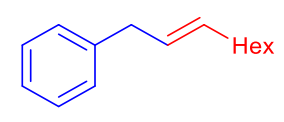

According to TP2, for this reaction $\mathrm{FeCl}_{2}(4.20 \mathrm{mg}, 33.0 \mu \mathrm{mol}, 10 \mathrm{~mol} \%),(E)$-1-iodooctene (3a, $79.0 \mathrm{mg}$, $333 \mu \mathrm{mol}, 1.00$ equiv) and freshly distilled THF $(0.30 \mathrm{~mL})$ were used. The reaction mixture was cooled to $0^{\circ} \mathrm{C}$ and benzylmanganese chloride $(1 \mathrm{c}, 1.18 \mathrm{~mL}, 400 \mu \mathrm{mol}, 1.20$ equiv, $0.34 \mathrm{M})$ was added dropwise before the mixture was allowed to warm to $25^{\circ} \mathrm{C}$ for $1 \mathrm{~h}$. Purification by flash column chromatography $\left(\mathrm{SiO}_{2}\right.$, isohexane, $\left.\mathrm{R}_{\mathrm{f}}=0.73\right)$ afforded the desired cross-coupling reaction product $4 \mathbf{d}(52.0 \mathrm{mg}, 256$ $\mu \mathrm{mol}, 77 \%)$ as a colorless oil.

${ }^{1} \mathrm{H}-\mathrm{NMR}\left(\mathbf{4 0 0} \mathbf{~ M H z}, \mathrm{CDCl}_{3}, \mathbf{p p m}\right) \delta=7.34-7.25(\mathrm{~m}, 2 \mathrm{H}), 7.23-7.17(\mathrm{~m}, 3 \mathrm{H}), 5.65-5.43(\mathrm{~m}, 2 \mathrm{H}), 3.34$ (d, $J=5.8 \mathrm{~Hz}, 2 \mathrm{H}), 2.07-1.97(\mathrm{~m}, 2 \mathrm{H}), 1.43-1.33(\mathrm{~m}, 2 \mathrm{H}), 1.30$ (ddd, $J=10.5,4.5,2.2 \mathrm{~Hz}, 6 \mathrm{H}), 0.93$ $-0.81(\mathrm{~m}, 3 \mathrm{H})$.

${ }^{13} \mathrm{C}-\mathrm{NMR}\left(101 \mathrm{MHz}, \mathrm{CDCl}_{3}, \mathrm{ppm}\right) \delta=141.3,132.3,128.8,128.6,128.4,126.0,39.2,32.7,31.9,29.6$, 29.0, 22.8, 14.3. 
IR $\left(\right.$ ATR, $\left.\mathbf{c m}^{-1}\right) \tilde{v}=2955,2926,2856,1741,1720,1706,1495,1453,965,746,696$.

MS (El, 70 eV, \%) m/z = 202 (10), 129 (12), 117 (71), 115 (28), 104 (100), 91 (43).

HRMS (EI, $70 \mathrm{eV}$ ) m/z: calc. for $\mathrm{C}_{15} \mathrm{H}_{22}$ : 202.1722; found 202.1714 .

\section{Synthesis of (E)-1-bromo-2-(3-phenylprop-1-en-1-yl)benzene (4e)}

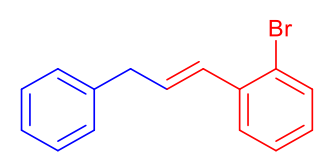

According to TP2, for this reaction $\mathrm{FeCl}_{2}(4.20 \mathrm{mg}, 33.0 \mu \mathrm{mol}, 10 \mathrm{~mol} \%)$, (E)-1-bromo-2-(2iodovinyl)benzene (3d, $103 \mathrm{mg}, 333 \mu \mathrm{mol}, 1.00$ equiv) and freshly distilled THF $(0.30 \mathrm{~mL})$ were used. The reaction mixture was cooled to $0^{\circ} \mathrm{C}$ and benzylmanganese chloride $(1 \mathrm{c}, 1.18 \mathrm{~mL}, 400 \mu \mathrm{mol}, 1.20$ equiv, $0.34 \mathrm{M}$ ) was added dropwise before the mixture was allowed to warm to $25^{\circ} \mathrm{C}$ for $1 \mathrm{~h}$. Purification by flash column chromatography $\left(\mathrm{SiO}_{2}\right.$, isohexane, $\left.\mathrm{R}_{\mathrm{f}}=0.54\right)$ afforded the desired cross-coupling reaction product $4 \mathrm{e}(79.0 \mathrm{mg}, 289 \mu \mathrm{mol}, 87 \%)$ as a colorless oil.

${ }^{1} \mathrm{H}-\mathrm{NMR}\left(400 \mathrm{MHz}, \mathrm{CDCl}_{3}, \mathrm{ppm}\right) \delta=7.50$ (ddd, $\left.J=17.3,7.9,1.5 \mathrm{~Hz}, 2 \mathrm{H}\right), 7.36-7.28(\mathrm{~m}, 2 \mathrm{H}), 7.28-$ $7.19(\mathrm{~m}, 4 \mathrm{H}), 7.05(\mathrm{td}, J=7.7,1.7 \mathrm{~Hz}, 1 \mathrm{H}), 6.81(\mathrm{dt}, J=15.6,1.6 \mathrm{~Hz}, 1 \mathrm{H}), 6.28(\mathrm{dt}, J=15.6,7.0 \mathrm{~Hz}$, $1 \mathrm{H}), 3.59(\mathrm{dd}, J=7.1,1.5 \mathrm{~Hz}, 2 \mathrm{H})$.

${ }^{13} \mathrm{C}-\mathrm{NMR}\left(101 \mathrm{MHz}, \mathrm{CDCl}_{3}, \mathrm{ppm}\right) \delta=139.9,137.4,133.0,132.4,130.1,128.8,128.7,128.5,127.5$, 127.1, 126.4, 123.4, 39.6.

IR $\left(\right.$ ATR, $\left.\mathbf{~ c m}^{-1}\right) \tilde{v}=3408,3062,3028,1696,1587,1496,1467,1454,1438,1265,1200,1023,964,745$, 697.

MS (EI, 70 eV, \%) m/z = 272 (16), 193 (100), 191 (25), 189 (15), 178 (73), 165 (22), 115 (98), 91 (11).

HRMS (El, $\mathbf{7 0} \mathrm{eV}$ ) m/z: calc. for $\mathbf{C}_{15} \mathbf{H}_{13} \mathrm{Br}$ : 272.0201; found 272.0196 .

\section{Synthesis of (3-(4-(tert-butyl)phenyl)prop-1-ene-1,1-diyl)dibenzene (4f)}<smiles>PC(=CCc1ccc(Br)cc1)Pc1ccccc1</smiles>

According to TP2, for this reaction $\mathrm{FeCl}_{2}(4.20 \mathrm{mg}, 33.0 \mu \mathrm{mol}, 10 \mathrm{~mol} \%)$, 2,2-diphenylvinyl trifluoromethanesulfonate $(3 \mathrm{e}, 109 \mathrm{mg}, 333 \mu \mathrm{mol}, 1.00$ equiv) and freshly distilled THF $(0.30 \mathrm{~mL})$ were used. The reaction mixture was cooled to $0^{\circ} \mathrm{C}$ and 4 -Tertbutylbenzylmanganese chloride $(\mathbf{1 d}, 1.08 \mathrm{~mL}$, $400 \mu \mathrm{mol}, 1.20$ equiv, $0.37 \mathrm{M}$ ) was added dropwise before the mixture was allowed to warm to $25^{\circ} \mathrm{C}$ for $12 \mathrm{~h}$. Purification by flash column chromatography $\left(\mathrm{SiO}_{2}\right.$, isohexane, $\left.\mathrm{R}_{\mathrm{f}}=0.59\right)$ afforded the desired cross-coupling reaction product $\mathbf{4 f}$ (103 $\mathrm{mg}, 315 \mu \mathrm{mol}, 95 \%)$ as a colorless oil.

${ }^{1} \mathrm{H}-\mathrm{NMR}\left(\mathbf{4 0 0} \mathrm{MHz}, \mathrm{CDCl}_{3}, \mathrm{ppm}\right) \delta=7.39$ (dd, $\left.J=7.1,1.2 \mathrm{~Hz}, 2 \mathrm{H}\right), 7.33$ (dd, $J=7.9,1.9 \mathrm{~Hz}, 3 \mathrm{H}$ ), 7.28 $-7.21(\mathrm{~m}, 7 \mathrm{H}), 7.16(\mathrm{~d}, J=8.3 \mathrm{~Hz}, 2 \mathrm{H}), 6.29(\mathrm{t}, J=7.6 \mathrm{~Hz}, 1 \mathrm{H}), 3.46(\mathrm{~d}, J=7.6 \mathrm{~Hz}, 2 \mathrm{H}), 1.32(\mathrm{~s}, 9 \mathrm{H})$. 
${ }^{13} \mathrm{C}-\mathrm{NMR}\left(101 \mathrm{MHz}, \mathrm{CDCl}_{3}, \mathrm{ppm}\right) \delta=148.9,142.6,142.4,140.0,138.0,130.1,128.4,128.2(2 \mathrm{C}), 128.1$, $127.5,127.2,127.1,125.5,35.5,34.5,31.5$.

IR $\left(\mathbf{A T R}, \mathbf{c m}^{-1}\right) \tilde{\mathrm{v}}=2961,2868,1699,1661,1606,1600,1514,1494,1446,1363,1318,1276,1176$, 1109, 1026, 1019, 829, 762, 697.

MS (El, 70 eV, \%) m/z = 326 (13), 269 (100), 191 (87), 178 (15), 115 (12), 91 (15).

HRMS (EI, $70 \mathrm{eV}$ ) m/z: calc. for $\mathrm{C}_{25} \mathrm{H}_{26}$ : 326.2035; found 326.2024.

\section{Synthesis of (E)-1-(4,4-diethoxybut-2-en-1-yl)-4-isopropylbenzene $(4 \mathrm{~g})$}<smiles>CCCc1ccc(CC=CC(OCC)OCC)cc1</smiles>

According to TP2, for this reaction $\mathrm{FeCl}_{2}(4.20 \mathrm{mg}, 33.0 \mu \mathrm{mol}, 10 \mathrm{~mol} \%),(E)$-1-bromo-3,3-diethoxyprop1-ene $(\mathbf{3 f}, 70.0 \mathrm{mg}, 333 \mu \mathrm{mol}, 1.00$ equiv) and freshly distilled THF $(0.30 \mathrm{~mL})$ were used. The reaction mixture was cooled to $0^{\circ} \mathrm{C}$ and 4-Isopropylbenzylmanganese chloride (1e, $1.08 \mathrm{~mL}, 400 \mu \mathrm{mol}, 1.20$ equiv, $0.37 \mathrm{M}$ ) was added dropwise before the mixture was allowed to warm to $25^{\circ} \mathrm{C}$ for $12 \mathrm{~h}$. Purification by flash column chromatography $\left(\mathrm{SiO}_{2}\right.$, isohexane:triethylamine $\left.=97: 3, \mathrm{Rf}_{\mathrm{f}}=0.50\right)$ afforded the desired cross-coupling reaction product $\mathbf{4 g}(69.0 \mathrm{mg}, 263 \mu \mathrm{mol}, 79 \%)$ as a colorless oil.

${ }^{1} \mathrm{H}$-NMR (400 MHz, $\mathrm{CDCl}_{3}$, ppm) $\delta=7.17-7.09(\mathrm{~m}, 4 \mathrm{H}), 5.27(\mathrm{dt}, J=1.9,0.9 \mathrm{~Hz}, 1 \mathrm{H}), 4.87(\mathrm{q}, J=1.7$ $\mathrm{Hz}, 1 \mathrm{H}), 4.73(\mathrm{~s}, 1 \mathrm{H}), 3.52(\mathrm{ddq}, J=60.2,9.4,7.1 \mathrm{~Hz}, 4 \mathrm{H}), 3.37(\mathrm{~d}, J=1.4 \mathrm{~Hz}, 2 \mathrm{H}), 2.88(\mathrm{p}, J=6.9 \mathrm{~Hz}$, $1 \mathrm{H}), 1.23(\mathrm{dd}, J=8.2,7.0 \mathrm{~Hz}, 12 \mathrm{H})$.

${ }^{13} \mathrm{C}-\mathrm{NMR}\left(\mathbf{1 0 1} \mathrm{MHz}, \mathrm{CDCl}_{3}, \mathrm{ppm}\right) \delta=146.7,146.1,136.6,129.4,126.4,114.5,102.6,61.6,37.5,33.8$, 24.2, 15.3 .

IR $\left(\right.$ ATR, $\left.\mathbf{c m}^{-1}\right) \tilde{v}=2962,2928,2873,1513,1326,1116,1098,1052,1020,1008,979,915,849,808$.

MS (El, $70 \mathrm{eV}, \%) \mathrm{m} / \mathrm{z}=173$ (100), 145 (60), 129 (27), 117 (15).

HRMS (El, $70 \mathrm{eV}$ ) m/z: calc. for $\mathbf{C}_{17} \mathrm{H}_{26} \mathbf{O}_{2}: 262.1933$; found 262.1924 .

\section{Synthesis of 1-((2-bromocyclopent-1-en-1-yl)methyl)-4-isopropylbenzene (4h)}<smiles>CCCc1ccc(CC2=C(Br)CCC2)cc1</smiles>

Adapted from TP2 (1.00 equiv of benzylic manganese chloride instead of 1.20 equiv); for this reaction $\mathrm{FeCl}_{2}$ (4.20 mg, $\left.33.0 \mu \mathrm{mol}, 10 \mathrm{~mol} \%\right), 1,2$-dibromocyclopent-1-ene $(\mathbf{3 g}, 75.0 \mathrm{mg}, 333 \mu \mathrm{mol}, 1.00$ equiv) and freshly distilled THF $(0.30 \mathrm{~mL})$ were used. The reaction mixture was cooled to $0{ }^{\circ} \mathrm{C}$ and $4-$ Isopropylbenzylmanganese chloride (1e, $0.90 \mathrm{~mL}, 333 \mu \mathrm{mol}, 1.00$ equiv, $0.37 \mathrm{M})$ was added dropwise before the mixture was allowed to warm to $25^{\circ} \mathrm{C}$ for $12 \mathrm{~h}$. Purification by flash column chromatography $\left(\mathrm{SiO}_{2}\right.$, isohexane, $\left.\mathrm{Rf}_{\mathrm{f}}=0.73\right)$ afforded the desired cross-coupling reaction product $4 \mathrm{~h}(54.0 \mathrm{mg}, 193$ $\mu \mathrm{mol}, 58 \%)$ as a colorless oil.

${ }^{1} \mathrm{H}-\mathrm{NMR}\left(400 \mathrm{MHz}, \mathrm{CDCl}_{3}, \mathrm{ppm}\right) \delta=7.18-7.10(\mathrm{~m}, 4 \mathrm{H}), 3.46(\mathrm{~s}, 2 \mathrm{H}), 2.89(\mathrm{p}, J=6.9 \mathrm{~Hz}, 1 \mathrm{H}), 2.67(\mathrm{t}$, $J=7.2 \mathrm{~Hz}, 2 \mathrm{H}), 2.23(\mathrm{t}, J=7.1 \mathrm{~Hz}, 2 \mathrm{H}), 1.90(\mathrm{p}, J=7.5 \mathrm{~Hz}, 2 \mathrm{H}), 1.25(\mathrm{~d}, J=6.8 \mathrm{~Hz}, 6 \mathrm{H})$. 
${ }^{13} \mathrm{C}-\mathrm{NMR}\left(101 \mathrm{MHz}, \mathrm{CDCl}_{3}, \mathrm{ppm}\right) \delta=146.8,140.1,136.1,128.6,126.6,116.6,40.1,36.1,33.8(2 \mathrm{C})$, 24.2, 21.7.

IR $\left(\mathbf{A T R}, \mathbf{c m}^{-1}\right) \tilde{\mathbf{v}}=2959,2925,2868,1710,1622,1512,1463,1431,1420,1315,1176,1082,1054$, $1020,935,841,817,808$.

MS (El, 70 eV, \%) m/z = 278 (10), 199 (70), 171 (29), 157 (55), 155 (43), 153 (16), 143 (29), 141 (18), 129 (100), 117 (13), 115 (23), 91 (19), 79 (13).

HRMS (El, $70 \mathrm{eV}$ ) m/z: calc. for $\mathbf{C}_{15} \mathrm{H}_{19} \mathrm{Br}$ : 278.0670; found 278.0647.

\section{Synthesis of ethyl 1-benzyl-4-(2-fluorobenzyl)-1,2,3,6-tetrahydropyridine (4i)}

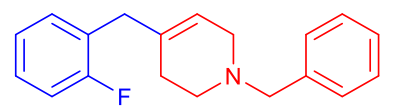

According to TP2, for this reaction $\mathrm{FeCl}_{2}(4.20 \mathrm{mg}, 33.0 \mu \mathrm{mol}, 10 \mathrm{~mol} \%)$, 1-benzyl-4-bromo-1,2,3,6tetrahydropyridine (3h, $84.0 \mathrm{mg}, 333 \mu \mathrm{mol}, 1.00$ equiv) and freshly distilled THF $(0.30 \mathrm{~mL})$ were used. The reaction mixture was cooled to $0^{\circ} \mathrm{C}$ and 2-Fluorobenzylmanganese chloride (1f, $1.00 \mathrm{~mL}, 400 \mu \mathrm{mol}$, 1.20 equiv, $0.40 \mathrm{M}$ ) was added dropwise before the mixture was allowed to warm to $25^{\circ} \mathrm{C}$ for $12 \mathrm{~h}$. Purification by flash column chromatography $\left(\mathrm{SiO}_{2}\right.$, isohexane:ethyl acetate $\left.=85: 15, \mathrm{R}_{\mathrm{f}}=0.35\right)$ afforded the desired cross-coupling reaction product $4 \mathbf{i}(79.0 \mathrm{mg}, 281 \mu \mathrm{mol}, 84 \%)$ as a yellowish oil.

${ }^{1} \mathrm{H}$-NMR (400 MHz, $\left.\mathrm{CDCl}_{3}, \mathbf{p p m}\right) \delta=7.30-7.21(\mathrm{~m}, 4 \mathrm{H}), 7.21-7.07(\mathrm{~m}, 3 \mathrm{H}), 7.03-6.90(\mathrm{~m}, 2 \mathrm{H})$, $5.29(\mathrm{dt}, J=3.3,1.7 \mathrm{~Hz}, 1 \mathrm{H}), 3.51(\mathrm{~s}, 2 \mathrm{H}), 3.25(\mathrm{~s}, 2 \mathrm{H}), 2.91(\mathrm{dt}, J=3.7,2.2 \mathrm{~Hz}, 2 \mathrm{H}), 2.49(\mathrm{t}, J=5.8$ $\mathrm{Hz}, 2 \mathrm{H}), 2.03(\mathrm{dt}, J=5.9,3.4 \mathrm{~Hz}, 2 \mathrm{H})$.

${ }^{13}$ C-NMR (101 MHz, CDCl 3, ppm) $\delta=161.4(\mathrm{~d}, J=245.2 \mathrm{~Hz}), 138.4,134.7,131.4(\mathrm{~d}, J=4.8 \mathrm{~Hz}), 129.4$, $128.3,127.9(\mathrm{~d}, J=8.1 \mathrm{~Hz}), 127.2,126.5(\mathrm{~d}, J=16.0 \mathrm{~Hz}), 124.0(\mathrm{~d}, J=3.6 \mathrm{~Hz}), 121.0,115.3(\mathrm{~d}, J=$ 22.3 Hz), 62.8, 53.0, 49.9, $36.0(\mathrm{~d}, J=2.9 \mathrm{~Hz}), 29.1$.

${ }^{19}$ F-NMR (377 MHz, $\left.\mathrm{CDCl}_{3}, \mathrm{ppm}\right) \delta=-118.2$.

IR $\left(\mathbf{A T R}, \mathbf{c m}^{-1}\right) \tilde{\mathrm{V}}=2908,2798,2749,1584,1491,1454,1364,1229,1149,1112,1094,1028,970,827$, $753,729,698$.

MS (El, 70 eV, \%) m/z = 172 (68), 109 (11), 91 (100).

HRMS (El, $70 \mathrm{eV}$ ) m/z: calc. for $\mathrm{C}_{19} \mathrm{H}_{20} \mathrm{FN}$ : 281.1580; found 281.1494 .

\section{Synthesis of (E)-1-fluoro-3-(non-2-en-1-yl)benzene (4j)}

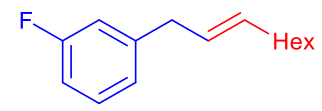

According to TP2, for this reaction $\mathrm{FeCl}_{2}(4.20 \mathrm{mg}, 33.0 \mu \mathrm{mol}, 10 \mathrm{~mol} \%),(E)$-1-iodooctene (3a, $79.0 \mathrm{mg}$, $333 \mu \mathrm{mol}, 1.00$ equiv) and freshly distilled THF $(0.30 \mathrm{~mL})$ were used. The reaction mixture was cooled to $0{ }^{\circ} \mathrm{C}$ and 3-fluorobenzylmanganese chloride $(\mathbf{1 g}, 1.33 \mathrm{~mL}, 400 \mu \mathrm{mol}, 1.20$ equiv, $0.30 \mathrm{M})$ was added dropwise before the mixture was allowed to warm to $25{ }^{\circ} \mathrm{C}$ for $1 \mathrm{~h}$. Purification by flash column 
chromatography $\left(\mathrm{SiO}_{2}\right.$, isohexane, $\left.\mathrm{R}_{\mathrm{f}}=0.80\right)$ afforded the desired cross-coupling reaction product $\mathbf{4 j}$ $(69.0 \mathrm{mg}, 313 \mu \mathrm{mol}, 94 \%)$ as a colorless oil.

${ }^{1} \mathrm{H}-\mathrm{NMR}\left(400 \mathrm{MHz}, \mathrm{CDCl}_{3}, \mathrm{ppm}\right) \delta=7.28-7.20(\mathrm{~m}, 1 \mathrm{H}), 6.96(\mathrm{~d}, J=7.7 \mathrm{~Hz}, 1 \mathrm{H}), 6.89(\mathrm{t}, J=8.4 \mathrm{~Hz}$, $2 \mathrm{H}), 5.60-5.44(\mathrm{~m}, 2 \mathrm{H}), 3.33(\mathrm{~d}, J=3.7 \mathrm{~Hz}, 2 \mathrm{H}), 2.04(\mathrm{dt}, J=10.4,6.2 \mathrm{~Hz}, 2 \mathrm{H}), 1.45-1.19(\mathrm{~m}, 8 \mathrm{H})$, $0.94-0.84(\mathrm{~m}, 3 \mathrm{H})$.

${ }^{13}$ C-NMR (101 MHz, $\mathrm{CDCl}_{3}$, ppm) $\delta=163.1(\mathrm{~d}, J=245.1 \mathrm{~Hz}), 143.9(\mathrm{~d}, J=7.1 \mathrm{~Hz}), 133.0,129.8(\mathrm{~d}, J$ $=8.3 \mathrm{~Hz}$ ), 128.0, $124.2(\mathrm{~d}, J=2.8 \mathrm{~Hz}), 115.4(\mathrm{~d}, J=21.0 \mathrm{~Hz}), 112.8(\mathrm{~d}, J=21.1 \mathrm{~Hz}), 38.9(\mathrm{~d}, J=1.7$ $\mathrm{Hz}), 32.7,31.9,29.5,29.0,22.8,14.2$.

${ }^{19} \mathrm{~F}-\mathrm{NMR}\left(377 \mathrm{MHz}, \mathrm{CDCl}_{3}, \mathrm{ppm}\right) \delta=-113.9$.

IR $\left(\mathbf{A T R}, \mathbf{~ c m}^{-1}\right) \tilde{v}=2956,2927,2856,1615,1586,1487,1467,1448,1266,1249,1139,966,948,871$, $778,754,723,685$.

MS (El, 70 eV, \%) m/z = 135 (27), 122 (100), 109 (18).

HRMS (El, $70 \mathrm{eV}$ ) m/z: calc. for $\mathrm{C}_{15} \mathrm{H}_{21} \mathrm{~F}: 220.1627$; found 220.1620.

\section{Synthesis of (3-(3-(trifluoromethyl)phenyl)prop-1-ene-1,1,2-triyl)tribenzene (4k)}<smiles>FC(F)(F)c1cccc(CC(=Cc2ccccc2)Pc2ccccc2)c1</smiles>

According to TP2, for this reaction $\mathrm{FeCl}_{2}(4.20 \mathrm{mg}, 33.0 \mu \mathrm{mol}, 10 \mathrm{~mol} \%$ ), (2-bromoethene-1,1,2triyl)tribenzene $(\mathbf{3 i}, 112 \mathrm{mg}, 333 \mu \mathrm{mol}, 1.00$ equiv) and freshly distilled THF $(0.30 \mathrm{~mL})$ were used. The reaction mixture was cooled to $0^{\circ} \mathrm{C}$ and 3 -(trifluoromethyl)benzylmanganese chloride $(\mathbf{1 h}, 1.29 \mathrm{~mL}, 400$ $\mu \mathrm{mol}, 1.20$ equiv, $0.31 \mathrm{M}$ ) was added dropwise before the mixture was allowed to warm to $25^{\circ} \mathrm{C}$ for 12 h. Purification by flash column chromatography $\left(\mathrm{SiO}_{2}\right.$, isohexane, $\left.\mathrm{R}_{\mathrm{f}}=0.18\right)$ afforded the desired crosscoupling reaction product $\mathbf{4 k}(120 \mathrm{mg}, 290 \mu \mathrm{mol}, 87 \%)$ as a white solid.

m.p. $\left({ }^{\circ} \mathbf{C}\right): 121-123$.

${ }^{1} \mathrm{H}-\mathrm{NMR}\left(400 \mathrm{MHz}, \mathrm{CDCl}_{3}, \mathrm{ppm}\right) \delta=7.39-7.23(\mathrm{~m}, 9 \mathrm{H}), 7.13-6.99(\mathrm{~m}, 8 \mathrm{H}), 6.97-6.93(\mathrm{~m}, 2 \mathrm{H})$, $3.92(\mathrm{~s}, 2 \mathrm{H})$.

${ }^{13}$ C-NMR (101 MHz, $\mathrm{CDCl}_{3}$, ppm) $\delta=143.0,142.6,142.5,141.7,141.2,137.2,132.1$ (d, $\left.J=1.1 \mathrm{~Hz}\right)$, $130.8,130.5(\mathrm{~d}, J=31.8 \mathrm{~Hz}), 130.0,129.4,128.7,128.5,128.1,127.7,127.2,126.6,126.3,125.7$ (q, $J$ $=3.7 \mathrm{~Hz}), 124.3(\mathrm{q}, J=272.9 \mathrm{~Hz}), 122.8(\mathrm{~d}, J=3.9 \mathrm{~Hz}), 41.4$.

${ }^{19}$ F-NMR (377 MHz, $\left.\mathrm{CDCl}_{3}, \mathrm{ppm}\right) \delta=-62.7$.

IR $\left(\mathbf{A T R}, \mathbf{c m}^{-1}\right) \tilde{V}=1489,1445,1330,1311,1281,1160,1154,1120,1095,1079,1031,1001,956,922$, $908,872,814,800,780,764,748,712,695,659$.

MS (El, 70 eV, \%) m/z = 414 (78), 336 (100), 323 (28), 283 (12), 267 (19), 265 (19), 259 (12), 255 (30), 252 (44), 239 (34), 191 (20), 189 (12), 178 (45), 176 (15), 167 (93), 165 (59), 152 (27), 126 (13).

HRMS (El, $70 \mathrm{eV}$ ) m/z: calc. for $\mathrm{C}_{28} \mathrm{H}_{21} \mathrm{~F}_{3}$ : 414.1595; found 414.1592 . 


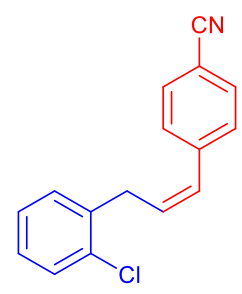

According to TP2, for this reaction $\mathrm{FeCl}_{2}(4.20 \mathrm{mg}, 33.0 \mu \mathrm{mol}, 10 \mathrm{~mol} \%)$, (Z)-4-(2bromovinyl)benzonitrile (3j, $70.0 \mathrm{mg}, 333 \mu \mathrm{mol}, 1.00$ equiv) and freshly distilled THF $(0.30 \mathrm{~mL})$ were used. The reaction mixture was cooled to $0^{\circ} \mathrm{C}$ and 2-Chlorobenzylmanganese chloride $(\mathbf{1 i}, 1.82 \mathrm{~mL}$, $400 \mu \mathrm{mol}, 1.20$ equiv, $0.22 \mathrm{M}$ ) was added dropwise before the mixture was allowed to warm to $25^{\circ} \mathrm{C}$ for $12 \mathrm{~h}$. Purification by flash column chromatography $\left(\mathrm{SiO}_{2}\right.$, isohexane:ethyl acetate $\left.=95: 5, \mathrm{Rf}_{\mathrm{f}}=0.30\right)$ afforded the desired cross-coupling reaction product $4 \mathbf{I}(83.0 \mathrm{mg}, 327 \mu \mathrm{mol}, 98 \%)$ as a white solid.

m.p. $\left({ }^{\circ} \mathbf{C}\right): 100-102$.

${ }^{1} \mathrm{H}-\mathrm{NMR}\left(400 \mathrm{MHz}, \mathrm{CDCl}_{3}\right.$, ppm) $\delta=7.69-7.58(\mathrm{~m}, 2 \mathrm{H}), 7.47-7.32(\mathrm{~m}, 3 \mathrm{H}), 7.25-7.12(\mathrm{~m}, 3 \mathrm{H})$, $6.62(\mathrm{dd}, J=11.6,1.9 \mathrm{~Hz}, 1 \mathrm{H}), 5.97(\mathrm{dt}, J=11.6,7.5 \mathrm{~Hz}, 1 \mathrm{H}), 3.73(\mathrm{dd}, J=7.5,1.8 \mathrm{~Hz}, 2 \mathrm{H})$.

${ }^{13} \mathrm{C}-\mathrm{NMR}\left(101 \mathrm{MHz}, \mathrm{CDCl}_{3}, \mathrm{ppm}\right) \delta=141.8,137.5,134.1,132.3(2 \mathrm{C}), 129.9,129.7,129.4(2 \mathrm{C}), 128.0$, 127.2, 119.1, 110.6, 32.7.

IR $\left(\mathbf{A T R}, \mathbf{c m}^{-1}\right) \tilde{v}=2230,1474,1215,1052,858,744,668$.

MS (El, 70 eV, \%) m/z = 253 (10), 218 (100), 216 (12), 203 (21), 190 (11), 140 (48), 115 (27).

HRMS (El, $70 \mathrm{eV}$ ) m/z: calc. for $\mathbf{C}_{16} \mathbf{H}_{12} \mathrm{CIN}$ : 253.0658; found 253.0651.

\section{Synthesis of (Z)-1-chloro-3-(3-(4-(trifluoromethyl)phenyl)allyl)benzene (4m)}<smiles>FC(F)(F)c1ccc(/C=C\c2cccc(Cl)c2)cc1</smiles>

According to TP2, for this reaction $\mathrm{FeCl}_{2}(4.20 \mathrm{mg}, 33.0 \mu \mathrm{mol}, 10 \mathrm{~mol} \%)$, (Z)-1-(2-iodovinyl)-4(trifluoromethyl)benzene (3k, $99.0 \mathrm{mg}, 333 \mu \mathrm{mol}, 1.00$ equiv) and freshly distilled THF $(0.30 \mathrm{~mL})$ were used. The reaction mixture was cooled to $0^{\circ} \mathrm{C}$ and 3-chlorobenzylmanganese chloride $(\mathbf{1 j}, 0.85 \mathrm{~mL}, 400$ $\mu \mathrm{mol}, 1.20$ equiv, $0.47 \mathrm{M}$ ) was added dropwise before the mixture was allowed to warm to $25^{\circ} \mathrm{C}$ for 1 h. Purification by flash column chromatography $\left(\mathrm{SiO}_{2}\right.$, isohexane, $\left.\mathrm{R}_{\mathrm{f}}=0.46\right)$ afforded the desired crosscoupling reaction product $4 \mathrm{~m}(97.0 \mathrm{mg}, 327 \mu \mathrm{mol}, 98 \%)$ as a colorless oil.

${ }^{1} \mathrm{H}-\mathrm{NMR}\left(400 \mathrm{MHz}, \mathrm{CDCl}_{3}, \mathrm{ppm}\right) \delta=7.61(\mathrm{~d}, J=8.1 \mathrm{~Hz}, 2 \mathrm{H}), 7.43-7.37(\mathrm{~m}, 2 \mathrm{H}), 7.26-7.19(\mathrm{~m}, 3 \mathrm{H})$, 7.09 (ddq, $J=7.4,1.5,0.8 \mathrm{~Hz}, 1 \mathrm{H}$ ), 6.65 (d, $J=11.6 \mathrm{~Hz}, 1 \mathrm{H}$ ), 5.94 (dt, $J=11.6,7.6 \mathrm{~Hz}, 1 \mathrm{H}$ ), 3.63 (dd, $J=7.6,1.8 \mathrm{~Hz}, 2 \mathrm{H})$. 
${ }^{13} \mathrm{C}$-NMR (101 MHz, CDCl 3 , ppm) $\delta=142.3,140.6(\mathrm{~d}, J=1.4 \mathrm{~Hz}), 134.6(2 \mathrm{C}), 131.8,130.0,129.6(2 \mathrm{C})$, $129.1(\mathrm{q}, J=32.5 \mathrm{~Hz}), 128.6,126.6(\mathrm{~d}, J=6.0 \mathrm{~Hz}), 125.4(\mathrm{q}, J=3.8 \mathrm{~Hz}), 124.32(\mathrm{~d}, J=271.9 \mathrm{~Hz}), 34.3$.

${ }^{19} \mathrm{~F}-\mathrm{NMR}\left(377 \mathrm{MHz}, \mathrm{CDCl}_{3}, \mathrm{ppm}\right) \delta=-62.5$.

IR $\left(\mathbf{A T R}, \mathbf{c m}^{-1}\right) \tilde{v}=1618,1597,1574,1476,1321,1161,1109,1080,1066,1016,849,778,747,734$, 684.

MS (El, 70 eV, \%) m/z = 296 (12), 261 (100), 246 (19), 227 (12), 221 (11), 192 (26), 189 (16), 183 (59), 149 (14), 115 (61).

HRMS (El, $70 \mathrm{eV}$ ) m/z: calc. for $\mathbf{C}_{16} \mathbf{H}_{12} \mathrm{ClF}_{3}: 296.0580$; found 296.0572.

\section{Synthesis of (3-(4-bromophenyl)prop-1-ene-1,1-diyl)dibenzene (4n)}<smiles>PC(=Cc1ccc(Br)cc1)c1ccccc1</smiles>

According to TP2, for this reaction $\mathrm{FeCl}_{2}(4.20 \mathrm{mg}, 33.0 \mu \mathrm{mol}, 10 \mathrm{~mol} \%)$, 2,2-diphenylvinyl trifluoromethanesulfonate (3e, $109 \mathrm{mg}, 333 \mu \mathrm{mol}, 1.00$ equiv) and freshly distilled THF $(0.30 \mathrm{~mL})$ were used. The reaction mixture was cooled to $0{ }^{\circ} \mathrm{C}$ and 4-Bromobenzylmanganese chloride $(\mathbf{1 k}, 1.82 \mathrm{~mL}$, $400 \mu \mathrm{mol}, 1.20$ equiv, $0.22 \mathrm{M}$ ) was added dropwise before the mixture was allowed to warm to $25^{\circ} \mathrm{C}$ for $12 \mathrm{~h}$. Purification by flash column chromatography $\left(\mathrm{SiO}_{2}\right.$, isohexane, $\left.\mathrm{R}_{\mathrm{f}}=0.39\right)$ afforded the desired cross-coupling reaction product $4 \mathrm{n}(50.0 \mathrm{mg}, 143 \mu \mathrm{mol}, 43 \%)$ as a colorless oil.

${ }^{1} \mathrm{H}-\mathrm{NMR}\left(400 \mathrm{MHz}, \mathrm{CDCl}_{3}\right.$, ppm) $\delta=7.42-7.35(\mathrm{~m}, 4 \mathrm{H}), 7.35-7.25(\mathrm{~m}, 2 \mathrm{H}), 7.25-7.19(\mathrm{~m}, 6 \mathrm{H}), 7.06$ (d, $J=8.4 \mathrm{~Hz}, 2 \mathrm{H}), 6.20$ (t, $J=7.6 \mathrm{~Hz}, 1 \mathrm{H}), 3.41(\mathrm{~d}, J=7.6 \mathrm{~Hz}, 2 \mathrm{H})$.

${ }^{13} \mathrm{C}-\mathrm{NMR}\left(101 \mathrm{MHz}, \mathrm{CDCl}_{3}, \mathrm{ppm}\right) \delta=143.1,142.3,140.0,139.7,131.6,130.3,129.9,128.5,128.3$, $127.5,127.4,127.3,127.0,119.9,35.4$.

IR $\left(\right.$ ATR, $\left.\mathbf{c m}^{-1}\right) \tilde{v}=3057,3026,2924,1710,1700,1659,1590,1486,1446,1402,1318,1277,1070$, $1011,800,754,696$.

MS (EI, 70 eV, \%) m/z = 348 (10), 272 (16), 270 (17), 269 (38), 192 (47), 191 (100), 190 (11), 189 (22), 178 (38), 165 (29), 91 (11).

HRMS (El, $70 \mathrm{eV}$ ) m/z: calc. for $\mathbf{C}_{21} \mathbf{H}_{17} \mathrm{Br}$ : 348.0514; found 348.0506 .

\section{Synthesis of (Z)-1-bromo-4-(3-(4-(trifluoromethyl)phenyl)allyl)benzene (40)}

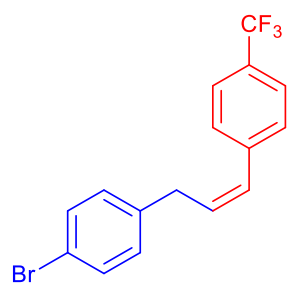

According to TP2, for this reaction $\mathrm{FeCl}_{2}(4.20 \mathrm{mg}, 33.0 \mu \mathrm{mol}, 10 \mathrm{~mol} \%)$, (Z)-1-(2-iodovinyl)-4(trifluoromethyl)benzene (3k, $99.0 \mathrm{mg}, 333 \mu \mathrm{mol}, 1.00$ equiv) and freshly distilled THF $(0.30 \mathrm{~mL})$ were 
used. The reaction mixture was cooled to $0^{\circ} \mathrm{C}$ and 4-Bromobenzylmanganese chloride $(\mathbf{1 k}, 1.82 \mathrm{~mL}$, $400 \mu \mathrm{mol}, 1.20$ equiv, $0.22 \mathrm{M}$ ) was added dropwise before the mixture was allowed to warm to $25^{\circ} \mathrm{C}$ for $1 \mathrm{~h}$. Purification by flash column chromatography $\left(\mathrm{SiO}_{2}\right.$, isohexane, $\left.\mathrm{Rf}=0.56\right)$ afforded the desired cross-coupling reaction product $\mathbf{4 0}(60.0 \mathrm{mg}, 176 \mu \mathrm{mol}, 53 \%)$ as a yellowish oil.

${ }^{1} \mathrm{H}-\mathrm{NMR}\left(400 \mathrm{MHz}, \mathrm{CDCl}_{3}, \mathrm{ppm}\right) \delta=7.60(\mathrm{~d}, J=8.2 \mathrm{~Hz}, 2 \mathrm{H}), 7.46-7.35(\mathrm{~m}, 4 \mathrm{H}), 7.11-7.04(\mathrm{~m}, 2 \mathrm{H})$, $6.63(\mathrm{~d}, J=11.5 \mathrm{~Hz}, 1 \mathrm{H}), 5.93(\mathrm{dt}, J=11.5,7.6 \mathrm{~Hz}, 1 \mathrm{H}), 3.60(\mathrm{dd}, J=7.6,1.7 \mathrm{~Hz}, 2 \mathrm{H})$.

${ }^{13}$ C-NMR (101 MHz, CDCl 3 , ppm) $\delta=140.6$ (d, $J=1.3 \mathrm{~Hz}$ ), 139.2, 132.1, 131.8, 130.1, 129.4, 129.1 (q, $J=32.5 \mathrm{~Hz}), 129.0,125.4(\mathrm{q}, J=3.8 \mathrm{~Hz}), 124.3(\mathrm{~d}, J=272.0 \mathrm{~Hz}), 120.2$, 34.1.

${ }^{19}$ F-NMR (377 MHz, $\left.\mathrm{CDCl}_{3}, \mathrm{ppm}\right) \delta=-62.5$.

IR $\left(\mathbf{A T R}, \mathbf{c m}^{-1}\right) \tilde{\mathrm{V}}=1618,1487,1322,1162,1120,1109,1066,1011,855,817,797,744$.

MS (El, 70 eV, \%) m/z = 340 (10), 261 (57), 246 (21), 221 (14), 191 (42), 189 (27), 183 (58), 171 (10), 169 (11), 165 (20), 159 (10), 151 (18), 133 (12), 115 (100), 102 (11), 91 (21), 89 (28), 69 (12).

HRMS (EI, $70 \mathrm{eV}$ ) m/z: calc. for $\mathrm{C}_{16} \mathrm{H}_{12} \mathrm{BrF}_{3}$ : 340.0074; found 340.0066 .

\section{Synthesis of ethyl (Z)-Ethyl 4-(3-methoxyphenyl)but-2-enoate (4p)}

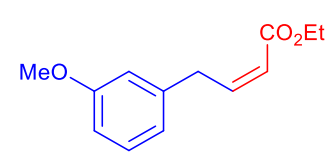

In relation to TP2, for this reaction $\mathrm{FeCl}_{2}(13.0 \mathrm{mg}, 100 \mu \mathrm{mol}, 10 \mathrm{~mol} \%)$, (Z)-ethyl 3-iodoacrylate (3I, 226 $\mathrm{mg}, 0.13 \mathrm{~mL}, 1.00 \mathrm{mmol}, 1.00$ equiv) and freshly distilled THF $(1.00 \mathrm{~mL})$ were used. The reaction mixture was cooled to $0^{\circ} \mathrm{C}$ and 3-methoxybenzylmanganese chloride $(1 \mathrm{a}, 3.08 \mathrm{~mL}, 1.20 \mathrm{mmol}, 1.20$ equiv, 0.39 M) was added dropwise before the mixture was allowed to warm to $25^{\circ} \mathrm{C}$ for $1 \mathrm{~h}$. Purification by flash column chromatography $\left(\mathrm{SiO}_{2}\right.$, isohexane:ethyl acetate $\left.=99: 1, \mathrm{R}_{\mathrm{f}}=0.23\right)$ afforded the desired crosscoupling reaction product $\mathbf{4 p}(218 \mathrm{mg}, 980 \mu \mathrm{mol}, 98 \%)$ as a colorless oil.

${ }^{1} \mathrm{H}-\mathrm{NMR}\left(400 \mathrm{MHz}, \mathrm{CDCl}_{3}, \mathbf{p p m}\right) \delta=7.22(\mathrm{t}, J=7.5 \mathrm{~Hz}, 1 \mathrm{H}), 6.89-6.71(\mathrm{~m}, 3 \mathrm{H}), 6.34(\mathrm{dt}, J=11.4,7.5$ $\mathrm{Hz}, 1 \mathrm{H}), 5.85(\mathrm{dt}, J=11.4,1.8 \mathrm{~Hz}, 1 \mathrm{H}), 4.22(\mathrm{q}, J=7.1 \mathrm{~Hz}, 2 \mathrm{H}), 4.00(\mathrm{dd}, J=7.5,1.7 \mathrm{~Hz}, 2 \mathrm{H}), 3.80(\mathrm{~s}$, $3 \mathrm{H}), 1.32(\mathrm{t}, \mathrm{J}=7.1 \mathrm{~Hz}, 3 \mathrm{H})$.

${ }^{13} \mathrm{C}-\mathrm{NMR}\left(101 \mathrm{MHz}, \mathrm{CDCl}_{3}, \mathrm{ppm}\right) \delta=166.5,160.0,147.9,141.2,129.7,121.1,120.1,114.5,111.9$, $60.1,55.3,35.3,14.4$.

IR $\left(\mathbf{A T R}, \mathbf{c m}^{-1}\right) \tilde{v}=2980,2938,2836,1714,1642,1600,1584,1490,1466,1454,1438,1410,1388$, 1314, 1284, 1258, 1198, 1160, 1148, 1112, 1094, 1038, 996, 932, 910, 862, 822, 780, 760, 696.

MS (El, 70 eV, \%) m/z = 220 (32), 174 (69), 163 (12), 160 (15), 147 (100), 145 (67), 131 (43), 117 (13), 115 (48), 103 (24), 91 (36), 77 (11).

HRMS (El, $70 \mathrm{eV}$ ) m/z: calc. for $\mathrm{C}_{13} \mathrm{H}_{16} \mathrm{O}_{3}: 220.1099$; found 220.1091 . 


\section{Synthesis of (Z)-ethyl 4-(4-(methylthio)phenyl)but-2-enoate (4q)}

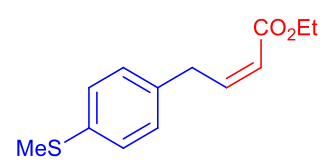

In relation to TP2, for this reaction $\mathrm{FeCl}_{2}(13.0 \mathrm{mg}, 100 \mu \mathrm{mol}, 10 \mathrm{~mol} \%)$, (Z)-ethyl 3-iodoacrylate (3I, 226 $\mathrm{mg}, 0.13 \mathrm{~mL}, 1.00 \mathrm{mmol}, 1.00$ equiv) and freshly distilled THF $(1.00 \mathrm{~mL})$ were used. The reaction mixture was cooled to $0{ }^{\circ} \mathrm{C}$ and 4-methylthiobenzylmanganese chloride $(\mathbf{1 b}, 3.33 \mathrm{~mL}, 1.20 \mathrm{mmol}, 1.20$ equiv, $0.36 \mathrm{M}$ ) was added dropwise before the mixture was allowed to warm to $25^{\circ} \mathrm{C}$ for $1 \mathrm{~h}$. Purification by flash column chromatography $\left(\mathrm{SiO}_{2}\right.$, isohexane:ethyl acetate $\left.=19: 1, \mathrm{R}_{\mathrm{f}}=0.56\right)$ afforded the desired cross-coupling reaction product $\mathbf{4 q}(180 \mathrm{mg}, 760 \mu \mathrm{mol}, 76 \%)$ as a pale-yellow oil.

${ }^{1} \mathrm{H}-N M R\left(400 \mathrm{MHz}, \mathrm{CDCl}_{3}\right.$, ppm) $\delta=7.24-7.19(\mathrm{~m}, 2 \mathrm{H}), 7.18-7.13(\mathrm{~m}, 2 \mathrm{H}), 6.31(\mathrm{dt}, J=11.4,7.6$ $\mathrm{Hz}, 1 \mathrm{H}$ ), $5.85(\mathrm{dt}, J=11.4,1.8 \mathrm{~Hz}, 1 \mathrm{H}), 4.21(\mathrm{q}, J=7.1 \mathrm{~Hz}, 2 \mathrm{H}), 3.98(\mathrm{dd}, J=7.6,1.7 \mathrm{~Hz}, 2 \mathrm{H}), 2.47(\mathrm{~s}$, $3 \mathrm{H}), 1.31(\mathrm{t}, J=7.1 \mathrm{~Hz}, 3 \mathrm{H})$.

${ }^{13} \mathrm{C}-\mathrm{NMR}\left(101 \mathrm{MHz}, \mathrm{CDCl}_{3}, \mathrm{ppm}\right) \delta=166.5,147.8,136.4(2 \mathrm{C}), 129.3,127.4,120.2,60.2,34.7,16.4$, 14.4 .

IR $\left(\right.$ ATR, $\left.\mathbf{c m}^{-1}\right) \tilde{\mathbf{v}}=2982,1494,806,1712,1642,1094,968,1018,734,1036,1114,1160,1192,1234$, 1296, 1386, 1412, 1438, 1464, 1478, 926, 912, 842, 2922, 722.

MS (EI, 70 eV, \%) m/z = 236 (54), 207 (15), 190 (82), 179 (21), 163 (17), 161 (100), 147 (18), 144 (27), 128 (21), 121 (11), 115 (78).

HRMS (EI, $70 \mathrm{eV}$ ) m/z: calc. for $\mathrm{C}_{13} \mathrm{H}_{16} \mathrm{O}_{2} \mathrm{~S}: 236.0871$; found 236.0864 .

\section{Synthesis of ethyl (E)-4-(4-(tert-butyl)phenyl)but-2-enoate (4r)}<smiles>CCOC(=O)C=CCc1ccc(Br)cc1</smiles>

According to TP2, for this reaction $\mathrm{FeCl}_{2}(4.20 \mathrm{mg}, 33.0 \mu \mathrm{mol}, 10 \mathrm{~mol} \%)$, ethyl $(E)$-3-iodoacrylate (3m, $76.0 \mathrm{mg}, 333 \mu \mathrm{mol}, 1.00$ equiv) and freshly distilled THF $(0.30 \mathrm{~mL})$ were used. The reaction mixture was cooled to $0{ }^{\circ} \mathrm{C}$ and 4-Tertbutylbenzylmanganese chloride ( $1 \mathrm{~d}, 1.08 \mathrm{~mL}, 400 \mu \mathrm{mol}, 1.20$ equiv, $0.37 \mathrm{M}$ ) was added dropwise before the mixture was allowed to warm to $25^{\circ} \mathrm{C}$ for $1 \mathrm{~h}$. Purification by flash column chromatography $\left(\mathrm{SiO}_{2}\right.$, isohexane:ethyl acetate $\left.=99: 1, \mathrm{R}_{\mathrm{f}}=0.26\right)$ afforded the desired crosscoupling reaction product $4 \mathbf{r}(45.0 \mathrm{mg}, 183 \mu \mathrm{mol}, 55 \%)$ as a colorless oil.

${ }^{1} \mathrm{H}-N M R\left(400 \mathrm{MHz}, \mathrm{CDCl}_{3}, \mathrm{ppm}\right) \delta=7.35(\mathrm{~s}, 1 \mathrm{H}), 7.33(\mathrm{~s}, 1 \mathrm{H}), 7.15-7.06(\mathrm{~m}, 3 \mathrm{H}), 5.82(\mathrm{dt}, J=15.5$, $1.7 \mathrm{~Hz}, 1 \mathrm{H}), 4.18(\mathrm{q}, J=7.1 \mathrm{~Hz}, 2 \mathrm{H}), 3.49(\mathrm{dd}, J=6.9,1.6 \mathrm{~Hz}, 2 \mathrm{H}), 1.36-1.27(\mathrm{~m}, 12 \mathrm{H})$.

${ }^{13} \mathrm{C}-\mathrm{NMR}\left(101 \mathrm{MHz}, \mathrm{CDCl}_{3}, \mathrm{ppm}\right) \delta=166.7,149.7,147.7,134.8,128.6,125.7,122.3,60.4,38.1,34.6$, $31.5,14.4$.

IR $\left(\mathbf{A T R}, \mathbf{c m}^{-1}\right) \tilde{v}=2962,2906,2870,1717,1655,1513,1464,1366,1320,1265,1196,1154,1110$, $1040,984,842,828,815,803,715$. 
MS (El, 70 eV, \%) m/z = 246 (26), 231 (100), 203 (24), 185 (61), 157 (49), 144 (30), 129 (19), 117 (19), 115 (17).

HRMS (El, $70 \mathrm{eV}$ ) m/z: calc. for $\mathrm{C}_{16} \mathrm{H}_{22} \mathrm{O}_{2}: 246.1620$; found 246.1612 .

\section{Synthesis of ethyl (Z)-4-(4-isopropylphenyl)-3-methylbut-2-enoate (4s)}<smiles>CCOC(=CC(C)=CCc1ccc(C(C)C)cc1)CC</smiles>

According to TP2, for this reaction $\mathrm{FeCl}_{2}(4.20 \mathrm{mg}, 33.0 \mu \mathrm{mol}, 10 \mathrm{~mol} \%)$, ethyl (Z)-3-iodobut-2-enoate (3n, $80.0 \mathrm{mg}, 333 \mu \mathrm{mol}, 1.00$ equiv) and freshly distilled THF $(0.30 \mathrm{~mL})$ were used. The reaction mixture was cooled to $0^{\circ} \mathrm{C}$ and 4-Isopropylbenzylmanganese chloride (1e, $1.08 \mathrm{~mL}, 400 \mu \mathrm{mol}, 1.20$ equiv, 0.37 M) was added dropwise before the mixture was allowed to warm to $25^{\circ} \mathrm{C}$ for $1 \mathrm{~h}$. Purification by flash column chromatography $\left(\mathrm{SiO}_{2}\right.$, isohexane:ethyl acetate $\left.=98: 2, \mathrm{R}_{\mathrm{f}}=0.27\right)$ afforded the desired crosscoupling reaction product $4 \mathrm{~s}(64.0 \mathrm{mg}, 260 \mu \mathrm{mol}, 78 \%)$ as a yellowish oil.

${ }^{1} \mathrm{H}-\mathrm{NMR}\left(400 \mathrm{MHz}, \mathrm{CDCl}_{3}\right.$, ppm) $\delta=7.21-7.05(\mathrm{~m}, 4 \mathrm{H}), 5.76(\mathrm{~d}, J=1.3 \mathrm{~Hz}, 1 \mathrm{H}), 4.19(\mathrm{~d}, J=7.1 \mathrm{~Hz}$, 2H), $3.98(\mathrm{~s}, 2 \mathrm{H}), 2.87(\mathrm{p}, J=6.9 \mathrm{~Hz}, 1 \mathrm{H}), 1.80(\mathrm{~d}, J=1.3 \mathrm{~Hz}, 3 \mathrm{H}), 1.30(\mathrm{t}, J=7.1 \mathrm{~Hz}, 3 \mathrm{H}), 1.23(\mathrm{~d}, J=$ $6.9 \mathrm{~Hz}, 6 \mathrm{H})$.

${ }^{13} \mathrm{C}-\mathrm{NMR}\left(101 \mathrm{MHz}, \mathrm{CDCl}_{3}, \mathrm{ppm}\right) \delta=166.7,158.1,146.9,136.3,129.0,126.6,117.0,59.8,38.6,33.8$, 24.8, 24.2, 14.5.

IR $\left(\mathbf{A T R}, \mathbf{c m}^{-1}\right) \tilde{\mathrm{v}}=2960,1712,1649,1512,1442,1376,1242,1161,1136,1097,1050,1020,864,844$ 800 .

MS (El, 70 eV, \%) m/z = 246 (10), 158 (100), 131 (24), 129 (36), 115 (14), 91 (12).

HRMS (El, $70 \mathrm{eV}$ ) m/z: calc. for $\mathrm{C}_{16} \mathrm{H}_{22} \mathrm{O}_{2}: 246.1620$; found 246.1613.

\section{Synthesis of (Z)-Ethyl 4-(2-fluorophenyl)but-2-enoate (4t)}<smiles>C=C(Cc1ccccc1F)OCC</smiles>

In relation to TP2, for this reaction $\mathrm{FeCl}_{2}(13.0 \mathrm{mg}, 100 \mu \mathrm{mol}, 10 \mathrm{~mol} \%)$, (Z)-ethyl 3-iodoacrylate (3I, 226 $\mathrm{mg}, 0.13 \mathrm{~mL}, 1.00 \mathrm{mmol}, 1.00$ equiv) and freshly distilled THF $(1.00 \mathrm{~mL})$ were used. The reaction mixture was cooled to $0^{\circ} \mathrm{C}$ and 2-fluorobenzylmanganese chloride (1f, $3.00 \mathrm{~mL}, 1.20 \mathrm{mmol}, 1.20$ equiv, 0.40 M) was added dropwise before the mixture was allowed to warm to $25^{\circ} \mathrm{C}$ for $1 \mathrm{~h}$. Purification by flash column chromatography $\left(\mathrm{SiO}_{2}\right.$, isohexane:ethyl acetate $\left.=19: 1, \mathrm{R}_{\mathrm{f}}=0.54\right)$ afforded the desired crosscoupling reaction product $\mathbf{4 t}(202 \mathrm{mg}, 970 \mu \mathrm{mol}, 97 \%)$ as a pale-yellow oil.

${ }^{1} \mathrm{H}-\mathrm{NMR}\left(400 \mathrm{MHz}, \mathrm{CDCl}_{3}, \mathrm{ppm}\right) \delta=7.41-7.17(\mathrm{~m}, 2 \mathrm{H}), 7.17-6.83(\mathrm{~m}, 2 \mathrm{H}), 6.36(\mathrm{dt}, J=11.4,7.5$ $\mathrm{Hz}, 1 \mathrm{H}), 5.89(\mathrm{dt}, J=11.4,1.8 \mathrm{~Hz}, 1 \mathrm{H}), 4.24(\mathrm{q}, J=7.1 \mathrm{~Hz}, 2 \mathrm{H}), 4.08(\mathrm{~d}, J=7.5 \mathrm{~Hz}, 2 \mathrm{H}), 1.34(\mathrm{t}, J=7.1$ $\mathrm{Hz}, 3 \mathrm{H})$. 
${ }^{13}$ C-NMR (101 MHz, $\left.\mathrm{CDCl}_{3}, \mathrm{ppm}\right) \delta=166.4,161.2(\mathrm{~d}, J=245.6 \mathrm{~Hz}), 146.5,130.9(\mathrm{~d}, J=4.7 \mathrm{~Hz}), 128.3$ (d, $J=8.0 \mathrm{~Hz}$ ), 126.5 (d, $J=15.9 \mathrm{~Hz}), 124.3(\mathrm{~d}, J=3.6 \mathrm{~Hz}), 120.6,115.5(\mathrm{~d}, J=21.8 \mathrm{~Hz}), 60.2,28.7$ (d, $J=3.1 \mathrm{~Hz}), 14.4$.

${ }^{19} \mathrm{~F}$ NMR (375 MHz, $\left.\mathrm{CDCl}_{3}, \mathrm{ppm}\right) \delta=-118.2$.

IR $\left(\mathbf{A T R}, \mathbf{~ c m}^{-1}\right) \tilde{v}=1388,1586,1032,1098,818,846,1716,1646,2982,1492,1456,1412,754,1298$, 1286, 1230, 1198, 1180, 1162, 1114, 864.

MS (El, 70 eV, \%) m/z = 208 (38), 180 (27), 162 (66), 151 (21), 135 (75), 133 (100), 123 (10), 115 (36), 109 (23), 83 (15).

HRMS (EI, $70 \mathrm{eV}$ ) m/z: calc. for $\mathrm{C}_{12} \mathrm{H}_{13} \mathrm{O}_{2} \mathrm{~F}: 208.0900$; found 208.0891.

Synthesis of ethyl (E)-4-(2-fluorophenyl)but-2-enoate (4u)<smiles>CCOC(=O)C=CCc1ccccc1F</smiles>

According to TP2, for this reaction $\mathrm{FeCl}_{2}(4.20 \mathrm{mg}, 33.0 \mu \mathrm{mol}, 10 \mathrm{~mol} \%)$, ethyl (E)-3-iodoacrylate (3m, $76.0 \mathrm{mg}, 333 \mu \mathrm{mol}, 1.00$ equiv) and freshly distilled THF $(0.30 \mathrm{~mL})$ were used. The reaction mixture was cooled to $0^{\circ} \mathrm{C}$ and 2-Fluorobenzylmanganese chloride (1f, $1.00 \mathrm{~mL}, 400 \mu \mathrm{mol}, 1.20$ equiv, $0.40 \mathrm{M}$ ) was added dropwise before the mixture was allowed to warm to $25^{\circ} \mathrm{C}$ for $1 \mathrm{~h}$. Purification by flash column chromatography $\left(\mathrm{SiO}_{2}\right.$, isohexane:ethyl acetate $\left.=95: 5, \mathrm{R}_{\mathrm{f}}=0.29\right)$ afforded the desired cross-coupling reaction product $4 \mathrm{u}(46.0 \mathrm{mg}, 221 \mu \mathrm{mol}, 66 \%)$ as a colorless oil.

${ }^{1} \mathrm{H}-N M R\left(400 \mathrm{MHz}, \mathrm{CDCl}_{3}, \mathrm{ppm}\right) \delta=7.19-7.07(\mathrm{~m}, 2 \mathrm{H}), 7.06-6.94(\mathrm{~m}, 3 \mathrm{H}), 5.73(\mathrm{dtd}, J=15.6,1.7$, $0.6 \mathrm{~Hz}, 1 \mathrm{H}), 4.11$ (q, $J=7.1 \mathrm{~Hz}, 2 \mathrm{H}), 3.48(\mathrm{dt}, J=6.7,1.5 \mathrm{~Hz}, 2 \mathrm{H}), 1.21(\mathrm{t}, J=7.1 \mathrm{~Hz}, 3 \mathrm{H}$ ).

${ }^{13}$ C-NMR (101 MHz, CDCl 3, ppm) $\delta=166.5,161.1$ (d, $J=246.1 \mathrm{~Hz}$ ), 145.8, 131.0 (d, $\left.J=4.4 \mathrm{~Hz}\right), 128.7$ $(\mathrm{d}, J=8.1 \mathrm{~Hz}), 124.9(\mathrm{~d}, J=15.9 \mathrm{~Hz}), 124.4(\mathrm{~d}, J=3.7 \mathrm{~Hz}), 122.8,115.6(\mathrm{~d}, J=21.8 \mathrm{~Hz}), 60.5,31.7$ (d, $J=3.2 \mathrm{~Hz}), 14.4$.

${ }^{19} \mathrm{~F}-\mathrm{NMR}\left(377 \mathrm{MHz}, \mathrm{CDCl}_{3}, \mathrm{ppm}\right) \delta=-118.1$.

IR $\left(\mathbf{A T R}, \mathbf{c m}^{-1}\right) \tilde{\mathbf{v}}=2982,2932,1716,1654,1585,1492,1456,1368,1332,1267,1230,1202,1157$, $1098,1037,982,830,754,701$.

MS (EI, 70 eV, \%) m/z = 208 (36), 180 (37), 173 (15), 162 (58), 153 (14), 151 (11), 135 (100), 133 (51), 125 (12), 115 (36), 109 (15).

HRMS (El, $70 \mathrm{eV}$ ) m/z: calc. for $\mathrm{C}_{12} \mathrm{H}_{13} \mathrm{FO}_{2}$ : 208.0900; found 208.0889.

\section{Synthesis of (Z)-Ethyl 4-(3-fluorophenyl)but-2-enoate (4v)}<smiles>CCOC=CCc1cccc(F)c1</smiles>

In relation to TP2, for this reaction $\mathrm{FeCl}_{2}(13.0 \mathrm{mg}, 100 \mu \mathrm{mol}, 10 \mathrm{~mol} \%)$, (Z)-ethyl 3-iodoacrylate (3I, 226 $\mathrm{mg}, 0.13 \mathrm{~mL}, 1.00 \mathrm{mmol}, 1.00$ equiv) and freshly distilled THF $(1.00 \mathrm{~mL})$ were used. The reaction mixture 
was cooled to $0^{\circ} \mathrm{C}$ and 3-fluorobenzylmanganese chloride $(\mathbf{1 g}, 4.00 \mathrm{~mL}, 1.20 \mathrm{mmol}, 1.20$ equiv, 0.30 M) was added dropwise before the mixture was allowed to warm to $25^{\circ} \mathrm{C}$ for $1 \mathrm{~h}$. Purification by flash column chromatography $\left(\mathrm{SiO}_{2}\right.$, isohexane:ethyl acetate $\left.=19: 1, \mathrm{R}_{\mathrm{f}}=0.51\right)$ afforded the desired crosscoupling reaction product $\mathbf{4 v}(148 \mathrm{mg}, 710 \mu \mathrm{mol}, 71 \%)$ as a pale-yellow oil.

${ }^{1} \mathrm{H}-\mathrm{NMR}\left(400 \mathrm{MHz}, \mathrm{CDCl}_{3}, \mathrm{ppm}\right) \delta=7.49-7.21(\mathrm{~m}, 1 \mathrm{H}), 7.03(\mathrm{~d}, J=7.6 \mathrm{~Hz}, 1 \mathrm{H}), 6.94$ (ddd, $J=10.8$, 8.8, $2.0 \mathrm{~Hz}, 2 \mathrm{H}), 6.34(\mathrm{dt}, J=11.4,7.6 \mathrm{~Hz}, 1 \mathrm{H}), 5.90(\mathrm{dt}, J=11.4,1.8 \mathrm{~Hz}, 1 \mathrm{H}), 4.24(\mathrm{q}, J=7.1 \mathrm{~Hz}, 2 \mathrm{H})$, 4.05 (dd, $J=7.6,1.5 \mathrm{~Hz}, 2 \mathrm{H}), 1.34(\mathrm{t}, J=7.1 \mathrm{~Hz}, 3 \mathrm{H})$.

${ }^{13} \mathrm{C}-N M R\left(101 \mathrm{MHz}, \mathrm{CDCl}_{3}, \mathbf{p p m}\right) \delta=166.4,163.1$ (d, $\left.J=245.8 \mathrm{~Hz}\right), 146.9,142.1$ (d, $\left.J=7.3 \mathrm{~Hz}\right), 130.1$ (d, $J=8.3 \mathrm{~Hz}$ ), 124.4 (d, $J=2.8 \mathrm{~Hz}), 120.7,115.6$ (d, $J=21.3 \mathrm{~Hz}), 113.4(\mathrm{~d}, J=21.0 \mathrm{~Hz}$ ), 60.2, 34.9 (d, $J=1.9 \mathrm{~Hz}), 14.4$.

${ }^{19}$ F NMR (375 MHz, $\left.\mathrm{CDCl}_{3}, \mathrm{ppm}\right) \delta=-113.3$.

IR $\left(\mathbf{A T R}, \mathbf{c m}^{-1}\right) \tilde{\mathrm{V}}=1644,1388,1714,1616,862,1588,1488,952,1448,2984,1412,1028,1136,1162$, 1196, 1250, 1270, 1284, 1298, 1096, 1076, 1038, 1466, 926, 892, 824, 782, 766, 692.

MS (El, 70 eV, \%) m/z = 208 (34), 180 (13), 162 (50), 151 (16), 135 (100), 133 (93), 115 (28), 109 (19), $83(11)$.

HRMS (El, $70 \mathrm{eV}$ ) m/z: calc. for $\mathrm{C}_{12} \mathrm{H}_{13} \mathrm{O}_{2} \mathrm{~F}: 208.0900$; found 208.0892.

\section{Synthesis of ethyl (Z)-4-(3-fluorophenyl)-3-methylbut-2-enoate (4w)}<smiles>CCOC(=O)/C=C(/C)Cc1cccc(F)c1</smiles>

According to TP2, for this reaction $\mathrm{FeCl}_{2}(4.20 \mathrm{mg}, 33.0 \mu \mathrm{mol}, 10 \mathrm{~mol} \%)$, ethyl (Z)-3-iodobut-2-enoate (3n, $80.0 \mathrm{mg}, 333 \mu \mathrm{mol}, 1.00$ equiv) and freshly distilled THF $(0.30 \mathrm{~mL})$ were used. The reaction mixture was cooled to $0{ }^{\circ} \mathrm{C}$ and 3-fluorobenzylmanganese chloride $(1 \mathrm{~g}, 1.33 \mathrm{~mL}, 400 \mu \mathrm{mol}, 1.20$ equiv, $0.30 \mathrm{M})$ was added dropwise before the mixture was allowed to warm to $25^{\circ} \mathrm{C}$ for $1 \mathrm{~h}$. Purification by flash column chromatography $\left(\mathrm{SiO}_{2}\right.$, isohexane:ethyl acetate $\left.=99: 1, \mathrm{R}_{\mathrm{f}}=0.30\right)$ afforded the desired crosscoupling reaction product $\mathbf{4 w}(51.0 \mathrm{mg}, 230 \mu \mathrm{mol}, 69 \%)$ as a colorless oil.

${ }^{1} \mathrm{H}-\mathrm{NMR}\left(400 \mathrm{MHz}, \mathrm{CDCl}_{3}, \mathrm{ppm}\right) \delta=7.26-7.19(\mathrm{~m}, 1 \mathrm{H}), 7.02(\mathrm{~d}, J=7.6 \mathrm{~Hz}, 1 \mathrm{H}), 6.96(\mathrm{dt}, J=10.0$, $1.9 \mathrm{~Hz}, 1 \mathrm{H}), 6.90(\mathrm{td}, J=8.3,2.1 \mathrm{~Hz}, 1 \mathrm{H}), 5.83-5.77(\mathrm{~m}, 1 \mathrm{H}), 4.19(\mathrm{q}, J=7.1 \mathrm{~Hz}, 2 \mathrm{H}), 4.02(\mathrm{~s}, 2 \mathrm{H})$, $1.79(\mathrm{~d}, J=1.3 \mathrm{~Hz}, 3 \mathrm{H}), 1.30(\mathrm{t}, J=7.1 \mathrm{~Hz}, 3 \mathrm{H})$.

${ }^{13}$ C-NMR (101 MHz, $\mathbf{C D C l}_{3}$, ppm) $\delta=166.5,163.1$ (d, $\left.J=245.4 \mathrm{~Hz}\right), 156.8,141.5$ (d, $\left.J=7.4 \mathrm{~Hz}\right), 129.9$ $(\mathrm{d}, J=8.4 \mathrm{~Hz}), 124.8(\mathrm{~d}, J=2.8 \mathrm{~Hz}), 117.7,115.9(\mathrm{~d}, J=21.3 \mathrm{~Hz}), 113.3(\mathrm{~d}, J=21.2 \mathrm{~Hz}), 59.9,38.6$ (d, $J=1.6 \mathrm{~Hz}), 24.7,14.4$.

${ }^{19}$ F-NMR (377 MHz, $\left.\mathrm{CDCl}_{3}, \mathrm{ppm}\right) \delta=-113.6$.

IR $\left(\mathbf{A T R}, \mathbf{c m}^{-1}\right) \tilde{v}=2981,1709,1651,1614,1588,1486,1445,1371,1351,1243,1168,1136,1096$, 1075, 1050, 951, 859, 783, 760, 690 .

MS (EI, 70 eV, \%) m/z = 222 (10), 176 (100), 148 (57), 133 (21), 128 (10), 109 (20).

HRMS (EI, $70 \mathrm{eV}$ ) m/z: calc. for $\mathrm{C}_{13} \mathrm{H}_{15} \mathrm{FO}_{2}$ : 222.1056; found 222.1079. 


\section{Synthesis of (Z)-Ethyl 4-(3-(trifluoromethyl)phenyl)but-2-enoate (4x)}<smiles>C=C(/C=C\Cc1cccc(C(F)(F)F)c1)OCC</smiles>

In relation to TP2, for this reaction $\mathrm{FeCl}_{2}(13.0 \mathrm{mg}, 100 \mu \mathrm{mol}, 10 \mathrm{~mol} \%)$, (Z)-ethyl 3-iodoacrylate (3I, 226 $\mathrm{mg}, 0.13 \mathrm{~mL}, 1.00 \mathrm{mmol}, 1.00$ equiv) and freshly distilled THF $(1.00 \mathrm{~mL})$ were used. The reaction mixture was cooled to $0{ }^{\circ} \mathrm{C}$ and 3 -(trifluoromethyl)benzylmanganese chloride $(\mathbf{1 h}, 3.90 \mathrm{~mL}, 1.20 \mathrm{mmol}, 1.20$ equiv, $0.31 \mathrm{M}$ ) was added dropwise before the mixture was allowed to warm to $25^{\circ} \mathrm{C}$ for $1 \mathrm{~h}$. Purification by flash column chromatography $\left(\mathrm{SiO}_{2}\right.$, isohexane:ethyl acetate $\left.=99: 1, \mathrm{R}_{\mathrm{f}}=0.23\right)$ afforded the desired cross-coupling reaction product $\mathbf{4} \mathbf{x}(130 \mathrm{mg}, 500 \mu \mathrm{mol}, 50 \%)$ as a pale-yellow oil.

${ }^{1} \mathrm{H}-\mathrm{NMR}\left(400 \mathrm{MHz}, \mathrm{CDCl}_{3}, \mathrm{ppm}\right) \delta=7.83-7.35(\mathrm{~m}, 4 \mathrm{H}), 6.32(\mathrm{dt}, J=11.4,7.6 \mathrm{~Hz}, 1 \mathrm{H}), 5.91$ (dt, $J=$ $11.4,1.7 \mathrm{~Hz}, 1 \mathrm{H}), 4.23(\mathrm{q}, J=7.1 \mathrm{~Hz}, 2 \mathrm{H}), 4.09(\mathrm{dd}, J=7.6,1.6 \mathrm{~Hz}, 2 \mathrm{H}), 1.32(\mathrm{t}, J=7.1 \mathrm{~Hz}, 3 \mathrm{H})$.

${ }^{13}$ C-NMR (101 MHz, $\mathrm{CDCl}_{3}$, ppm) $\delta=166.3,146.5,140.5,132.2,131.1$ (q, $\left.J=32.1 \mathrm{~Hz}\right), 129.2,125.4$ (q, $J=3.8 \mathrm{~Hz}$ ), $124.3(\mathrm{q}, J=272.3 \mathrm{~Hz}), 123.4$ (q, $J=3.8 \mathrm{~Hz}), 121.0,60.3,34.9,14.4$.

${ }^{19} \mathrm{~F}$ NMR (375 MHz, $\left.\mathrm{CDCl}_{3}, \mathrm{ppm}\right) \delta=-62.6$.

IR $\left(\mathbf{A T R}, \mathbf{c m}^{-1}\right) \tilde{\mathbf{v}}=2984,1716,1646,1450,1412,1388,1330,1238,1194,1160,1120,1096,1072$ 1038, 1002, 936, 918, 900, 868, 822, 798, 762, 702, 656.

MS (El, 70 eV, \%) m/z = 258 (55), 230 (50), 213 (61), 210 (12), 201 (13), 193 (30), 185 (59), 183 (34) 181 (11), 175 (11), 173 (13), 165 (87), 164 (50), 161 (32), 159 (22), 146 (16), 133 (22), 115 (100), 109 (15).

HRMS (El, $70 \mathrm{eV}$ ) m/z: calc. for $\mathrm{C}_{13} \mathrm{H}_{13} \mathrm{O}_{2} \mathrm{~F}_{3}$ : 258.0868; found 258.0858 .

\section{Synthesis of (Z)-Ethyl 4-(2-chlorophenyl)but-2-enoate (4y)}<smiles>C=C(Cc1ccccc1Cl)OCC</smiles>

In relation to TP2, for this reaction $\mathrm{FeCl}_{2}(13.0 \mathrm{mg}, 100 \mu \mathrm{mol}, 10 \mathrm{~mol} \%)$, (Z)-ethyl 3-iodoacrylate (3I, 226 $\mathrm{mg}, 0.13 \mathrm{~mL}, 1.00 \mathrm{mmol}, 1.00$ equiv) and freshly distilled THF $(1.00 \mathrm{~mL})$ were used. The reaction mixture was cooled to $0{ }^{\circ} \mathrm{C}$ and 2-chlorobenzylmanganese chloride (1i, $5.45 \mathrm{~mL}, 1.20 \mathrm{mmol}, 1.20$ equiv, 0.22 M) was added dropwise before the mixture was allowed to warm to $25^{\circ} \mathrm{C}$ for $1 \mathrm{~h}$. Purification by flash column chromatography $\left(\mathrm{SiO}_{2}\right.$, isohexane:ethyl acetate $\left.=99: 1, \mathrm{R}_{\mathrm{f}}=0.26\right)$ afforded the desired crosscoupling reaction product $4 \mathbf{y}(113 \mathrm{mg}, 500 \mu \mathrm{mol}, 50 \%)$ as a pale-yellow oil.

${ }^{1} \mathrm{H}-\mathrm{NMR}\left(400 \mathrm{MHz}, \mathrm{CDCl}_{3}, \mathrm{ppm}\right) \delta=7.36(\mathrm{dd}, J=7.4,1.8 \mathrm{~Hz}, 1 \mathrm{H}), 7.30(\mathrm{dd}, J=7.2,2.1 \mathrm{~Hz}, 1 \mathrm{H}), 7.23$ $-7.13(\mathrm{~m}, 2 \mathrm{H}), 6.33$ (dt, $J=11.4,7.4 \mathrm{~Hz}, 1 \mathrm{H}), 5.88(\mathrm{dt}, J=11.4,1.8 \mathrm{~Hz}, 1 \mathrm{H}), 4.19$ (ddd, $J=10.8,9.2$, $4.5 \mathrm{~Hz}, 4 \mathrm{H}), 1.32(\mathrm{t}, J=7.1 \mathrm{~Hz}, 3 \mathrm{H})$.

${ }^{13}$ C-NMR (101 MHz, $\left.\mathrm{CDCl}_{3}, \mathrm{ppm}\right) \delta=166.4,146.3,137.4,134.2,130.8,129.6,128.0,127.2,120.7$, $60.2,33.1,14.4$. 
IR $\left(\mathbf{A T R}, \mathbf{c m}^{-1}\right) \tilde{v}=806,842,1410,750,1444,1714,1644,1474,680,668,1162,1192,1234,1286$, 1298, 1386, 1126, 1096, 1052, 1032, 926, 2928, 2982.

MS (EI, 70 eV, \%) m/z = 224 (51), 215 (16), 213 (11), 193 (12), 179 (100), 178 (45), 167 (11), 165 (19), 151 (16), 149 (19), 144 (13), 127 (23), 125 (75), 115 (39), 89 (11).

HRMS (EI, $70 \mathrm{eV}$ ) m/z: calc. for $\mathrm{C}_{12} \mathrm{H}_{13} \mathrm{O}_{2} \mathrm{Cl}$ : 224.0604; found 224.0614 .

\section{Synthesis of (Z)-Ethyl 4-(3-chlorophenyl)but-2-enoate (4z)}<smiles>CCOC(C)=CCc1cccc(Cl)c1</smiles>

In relation to TP2, for this reaction $\mathrm{FeCl}_{2}(13.0 \mathrm{mg}, 100 \mu \mathrm{mol}, 10 \mathrm{~mol} \%)$, (Z)-ethyl 3-iodoacrylate (3I, 226 $\mathrm{mg}, 0.13 \mathrm{~mL}, 1.00 \mathrm{mmol}, 1.00$ equiv) and freshly distilled THF $(1.00 \mathrm{~mL})$ were used. The reaction mixture was cooled to $0{ }^{\circ} \mathrm{C}$ and 3-chlorobenzylmanganese chloride $(\mathbf{1 j}, 2.55 \mathrm{~mL}, 1.20 \mathrm{mmol}, 1.20$ equiv, 0.47 M) was added dropwise before the mixture was allowed to warm to $25^{\circ} \mathrm{C}$ for $1 \mathrm{~h}$. Purification by flash column chromatography $\left(\mathrm{SiO}_{2}\right.$, isohexane:ethyl acetate $\left.=19: 1, \mathrm{R}_{f}=0.54\right)$ afforded the desired crosscoupling reaction product $\mathbf{4 z}(163 \mathrm{mg}, 730 \mu \mathrm{mol}, 73 \%)$ as a pale-yellow oil.

${ }^{1} \mathrm{H}-\mathrm{NMR}\left(400 \mathrm{MHz}, \mathrm{CDCl}_{3}, \mathrm{ppm}\right) \delta=7.42-7.18(\mathrm{~m}, 3 \mathrm{H}), 7.14(\mathrm{dd}, J=7.0,1.8 \mathrm{~Hz}, 1 \mathrm{H}), 6.32(\mathrm{dt}, J=$ $11.4,7.6 \mathrm{~Hz}, 1 \mathrm{H}), 5.90(\mathrm{dt}, J=11.4,1.7 \mathrm{~Hz}, 1 \mathrm{H}), 4.24(\mathrm{q}, J=7.1 \mathrm{~Hz}, 2 \mathrm{H}), 4.03(\mathrm{dd}, J=7.6,1.7 \mathrm{~Hz}, 2 \mathrm{H})$, $1.34(\mathrm{t}, J=7.1 \mathrm{~Hz}, 3 \mathrm{H})$.

${ }^{13} \mathrm{C}-\mathrm{NMR}\left(101 \mathrm{MHz}, \mathrm{CDCl}_{3}, \mathrm{ppm}\right) \delta=166.4,146.8,141.6,134.5,130.0,128.9,127.0,126.7,120.7$, $60.3,34.8,14.4$.

IR $\left(\mathbf{A T R}, \mathbf{c m}^{-1}\right) \tilde{\mathrm{v}}=1644,1598,1574,1388,780,2982,860,682,932,1714,1036,1080,1000,1094$, 1160, 1194, 1236, 1286, 1298, 1114, 1410, 1430, 1476, 886, 818, 756, 736, 702.

MS (EI, 70 eV, \%) m/z = 224 (28), 179 (23), 178 (35), 167 (16), 153 (18), 151 (53), 149 (30), 144 (25), 138 (14), 133 (10), 125 (14), 116 (22), 115 (100), 103 (12), 89 (16).

HRMS (El, $70 \mathrm{eV}$ ) m/z: calc. for $\mathrm{C}_{12} \mathrm{H}_{13} \mathrm{O}_{2} \mathrm{Cl}$ : 224.0604; found 224.0597. 


\section{Studies on the catalytically active species and catalytic cycle}

All the samples were prepared in a recirculating mBraun LabMaster DP inert atmosphere (Ar) drybox and vacuum Schlenk lines. Glassware was dried overnight at $60{ }^{\circ} \mathrm{C}$ before use. NMR spectra were obtained using a Bruker DPX $400 \mathrm{MHz}$ spectrometer. Chemical shifts for ${ }^{1} \mathrm{H}$ NMR spectra were referenced to solvent impurities (herein THF). NMR tubes equipped with a J. Young valve were used for all ${ }^{1} H$ NMR experiments. The GC-MS analysis was performed using $n$-decane as internal standard. The reactions were quenched with air and addition of distilled water. The organic products were extracted using DCM, and injected into the GC-MS

1 - Transmetallation of benzyl groups from $\mathrm{Bn}[\mathrm{Mn}] \mathrm{Cl}$ to the iron(II) precatalyst.

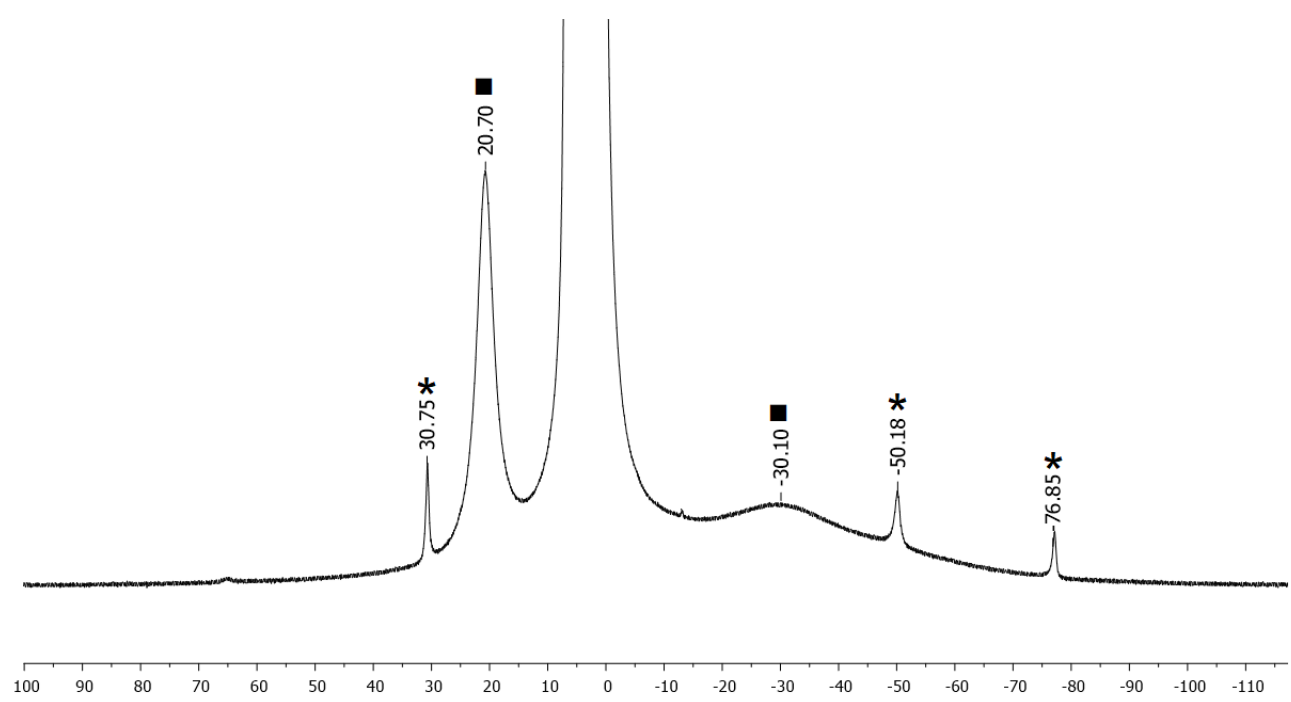

Figure $\mathrm{S} 1:{ }^{1} \mathrm{H}$ NMR spectrum (recorded at $25^{\circ} \mathrm{C}$ in $\mathrm{d}_{8}-\mathrm{THF}$ ) of a $0.08 \mathrm{M}$ solution of $\mathrm{BnMnCl}$ in the presence of 0.1 equiv $\mathrm{FeCl}_{2}\left({ }^{*}=\left[\mathrm{Bn}_{3} \mathrm{Fe}^{\mathrm{ll}}\right]^{-}: \delta=31 \mathrm{ppm}(6 \mathrm{H}, \mathrm{H}\right.$ meta), $-50 \mathrm{ppm}(6 \mathrm{H}$, Hortho), $-77 \mathrm{ppm}$ $(3 \mathrm{H}, \mathrm{Hpara}) ; \mathbf{-}=\mathrm{BnMnCl})$. 
$2-$ Reaction of in-situ-generated $\mathrm{Bn}[\mathrm{Mn}] \mathrm{Cl}$ with $\mathrm{TMSCH}=\mathrm{CHBr}$.

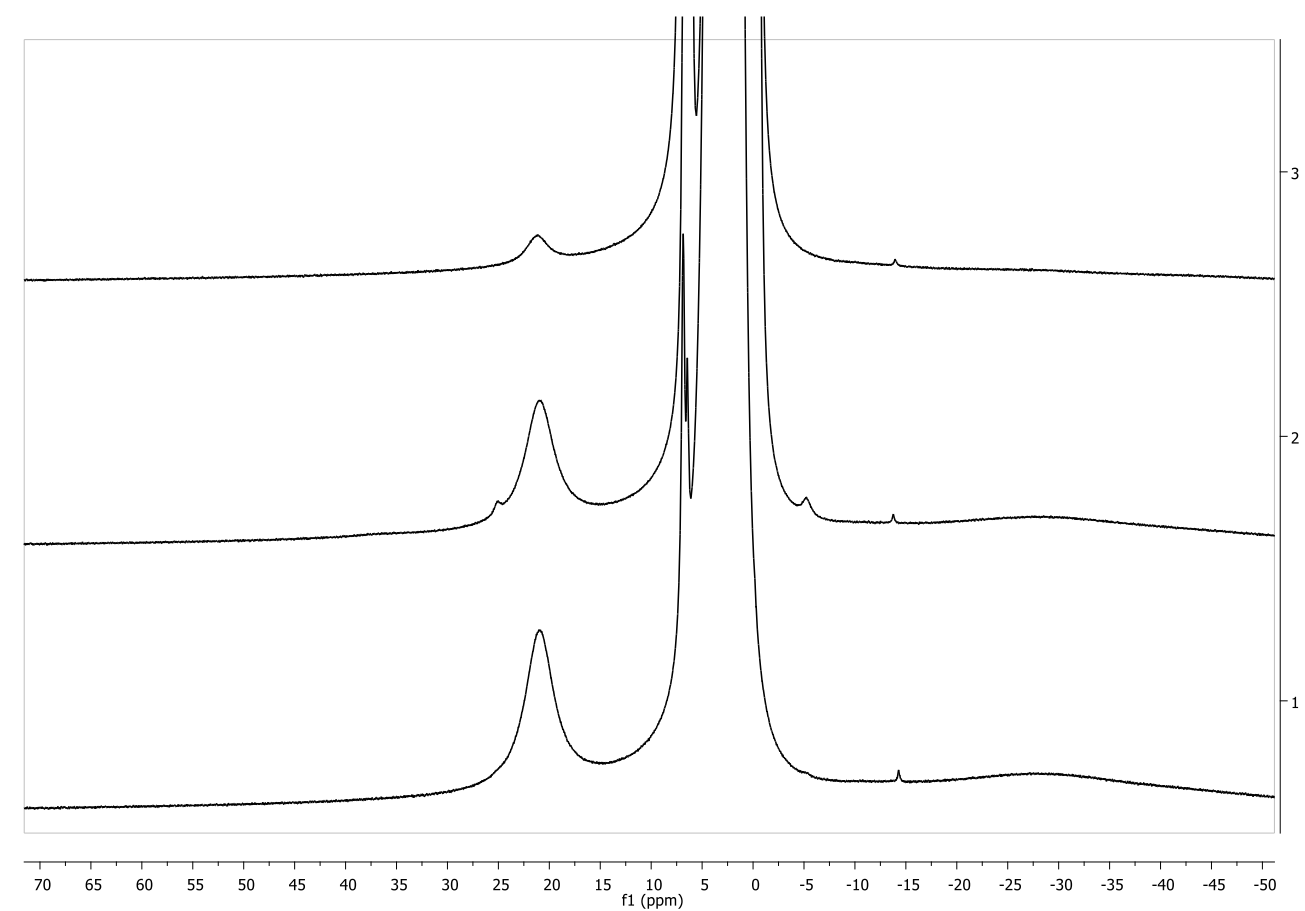

Figure S2: ${ }^{1} \mathrm{H}$ NMR spectra, recorded at $\mathrm{T}=25^{\circ} \mathrm{C}$, of a d8-THF solution of 1 ) $\mathrm{MnCl}_{2} \cdot 2 \mathrm{LiCl}+1$ equiv $\mathrm{BnMgCl}(0.08 \mathrm{M})$; followed by addition of 2) 1 equiv $\mathrm{TMSCH}=\mathrm{CHBr}$; 3) after $3 \mathrm{~h}$. All spectra were calibrated using mesitylene as an internal standard.

3 - Fe-catalyzed couplings between benzyl nucleophiles and $\mathrm{TMSCH}=\mathrm{CHBr}$.

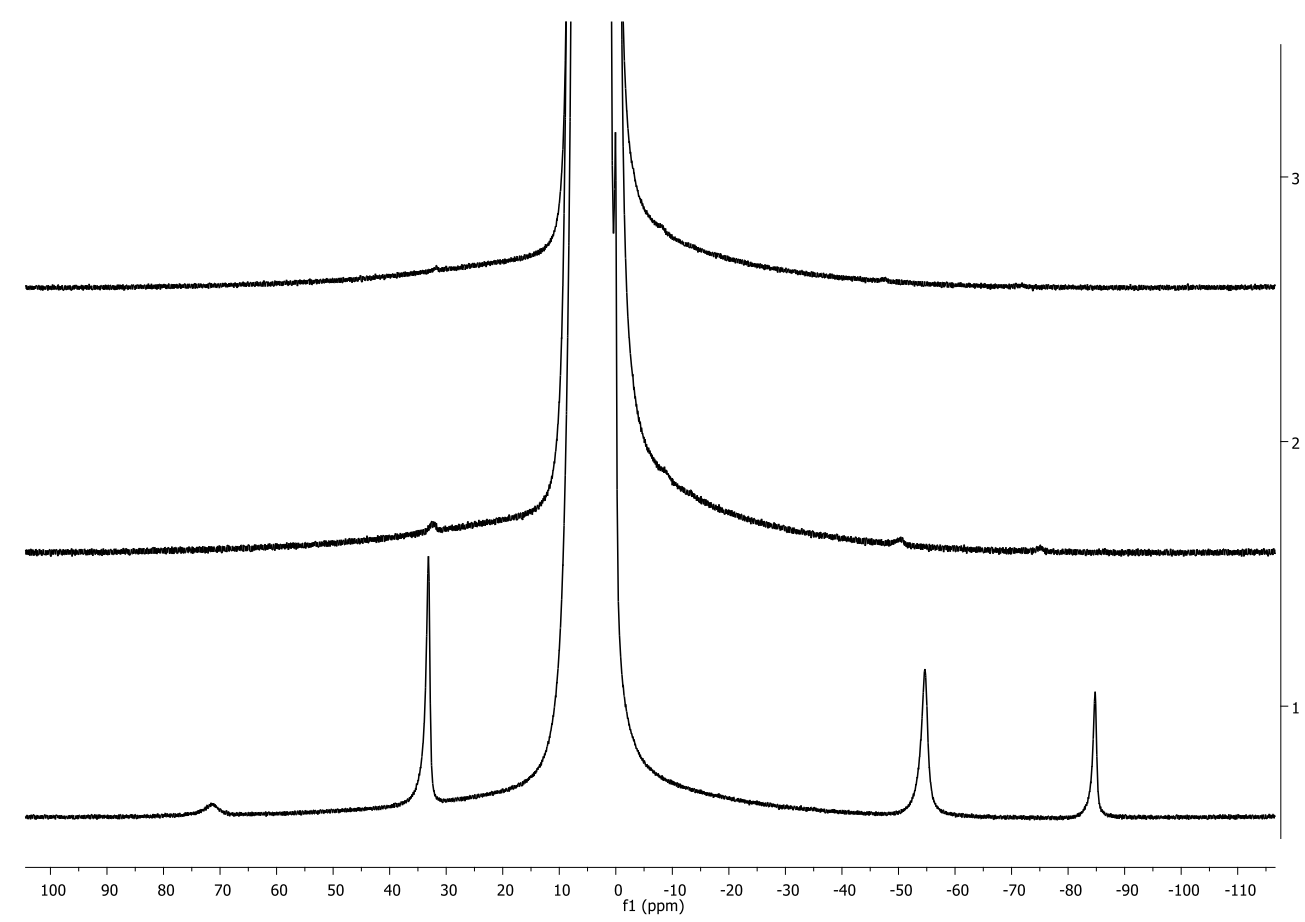

Figure S3 : ${ }^{1} \mathrm{H}$ NMR spectra of a d8-THF solution of 1$) \mathrm{FeCl}_{2}(0.08 \mathrm{M})+3 \mathrm{BnMgCl}$ (formation of [Bn $\left.{ }_{3} \mathrm{Fe}^{\prime \prime l}\right]-$ ) at $0{ }^{\circ} \mathrm{C}$, followed by direct addition at $0{ }^{\circ} \mathrm{C}$ of 2) 10 equiv of $\mathrm{TMSCH}=\mathrm{CHBr}$ (reaction time : 2 minutes); 3) after $5 \mathrm{~min}$ at $10^{\circ} \mathrm{C}$ (7 minutes total reaction time). All spectra were calibrated using the THF peaks. 


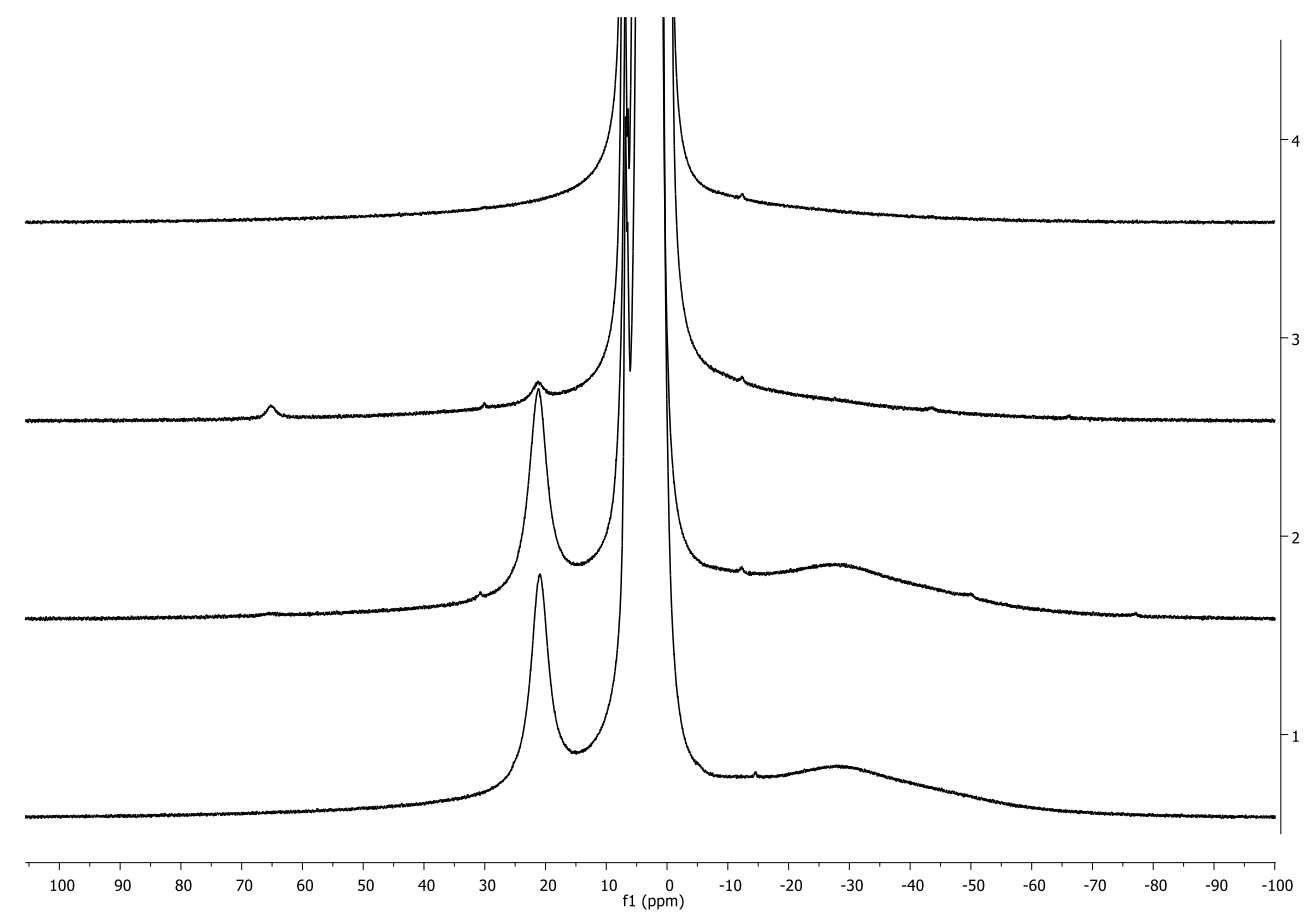

Figure S4 : ${ }^{1} \mathrm{H}$ NMR spectra, recorded at $\mathrm{T}=25^{\circ} \mathrm{C}$, of a d8-THF solution of 1$) \mathrm{Bn}[\mathrm{Mn}] \mathrm{Cl}(0.08 \mathrm{M})$, followed by direct addition of 2) 0.1 equiv of $\mathrm{FeCl}_{2}$ and 3) 1 equiv of $\mathrm{TMSCH}=\mathrm{CHBr}$; 4) after 10 minutes at RT. All spectra were calibrated using mesitylene as an internal standard.

a)

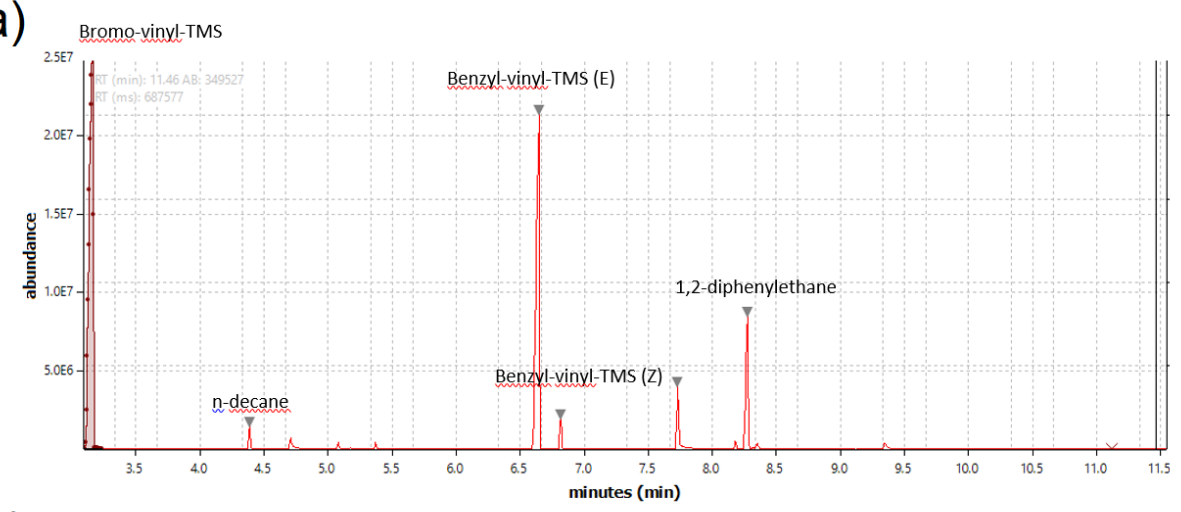

b)

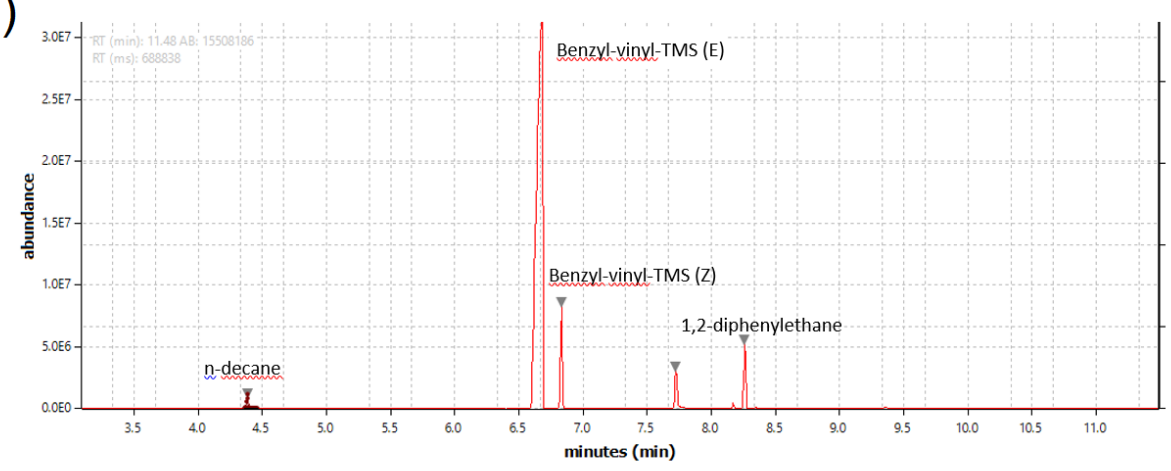

Figure S5: GC-MS analysis, after 30 min reaction time, of a) a solution of $\mathrm{BnMnCl}+1$ equiv $\mathrm{TMSCH}=\mathrm{CHBr}(0.08 \mathrm{M})$, in THF, after $30 \mathrm{~min}$ at $\mathrm{RT}$; b) same solution than a) but in the presence of 0.1 equiv $\mathrm{FeCl}_{2}$. 


\section{List of abbreviations}

$\begin{array}{ll}\text { acac } & \text { acetylacetonate } \\ \mathrm{Bu} O & \text { dibutyl ether } \\ \text { d } & \text { doublet } \\ \text { E } & \text { electrophile } \\ \text { equiv } & \text { equivalent } \\ \text { Et } & \text { ethyl } \\ \text { FG } & \text { functionalized group } \\ \text { GC } & \text { gas chromatography } \\ \text { h } & \text { hour } \\ \text { hept } & \text { heptuplet } \\ \mathrm{HRMS} & \text { high resolution mass spectrometry } \\ \mathrm{Hz} & \text { hertz } \\ \text { IPr } & \text { iso-propyl } \\ \mathrm{IR} & \text { infrared spectra } \\ \mathrm{m} & \text { multiplet } \\ \mathrm{Me} & \text { methyl } \\ \text { min } & \text { minute } \\ \text { m.p. } & \text { melting point } \\ \mathrm{MS} & \text { mass spectrometry } \\ \mathrm{MTBE} & \text { methyl tert-butyl ether } \\ \mathrm{NMR} & \text { nuclear magnetic resonance } \\ \mathrm{OMe} & \text { methoxy } \\ \mathrm{Ph} & \text { phenyl } \\ \text { ppm } & \text { parts per million } \\ \mathrm{q} & \text { quartet } \\ \mathrm{R} f & \text { retention factor } \\ \mathrm{S} & \text { singulet } \\ \mathrm{SMe} & \text { thiomethyl } \\ \mathrm{t} & \text { triplet } \\ \text { tBu } & \text { tert-butyl } \\ \text { THF } & \text { tetrahydrofuran } \\ \text { TLC } & \text { thin layer chromatography } \\ \mathrm{TMS} & \text { trimethylsilyl } \\ \text { TP } & \text { typical procedure } \\ \mathrm{UV} & \text { ultraviolet light } \\ & \end{array}$


NMR spectra of compounds $3 e$ and $4 a-z$

${ }^{1} \mathrm{H},{ }^{13} \mathrm{C}$ and ${ }^{19} \mathrm{~F}$ NMR spectra of compound $3 e$

葛

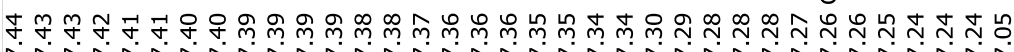
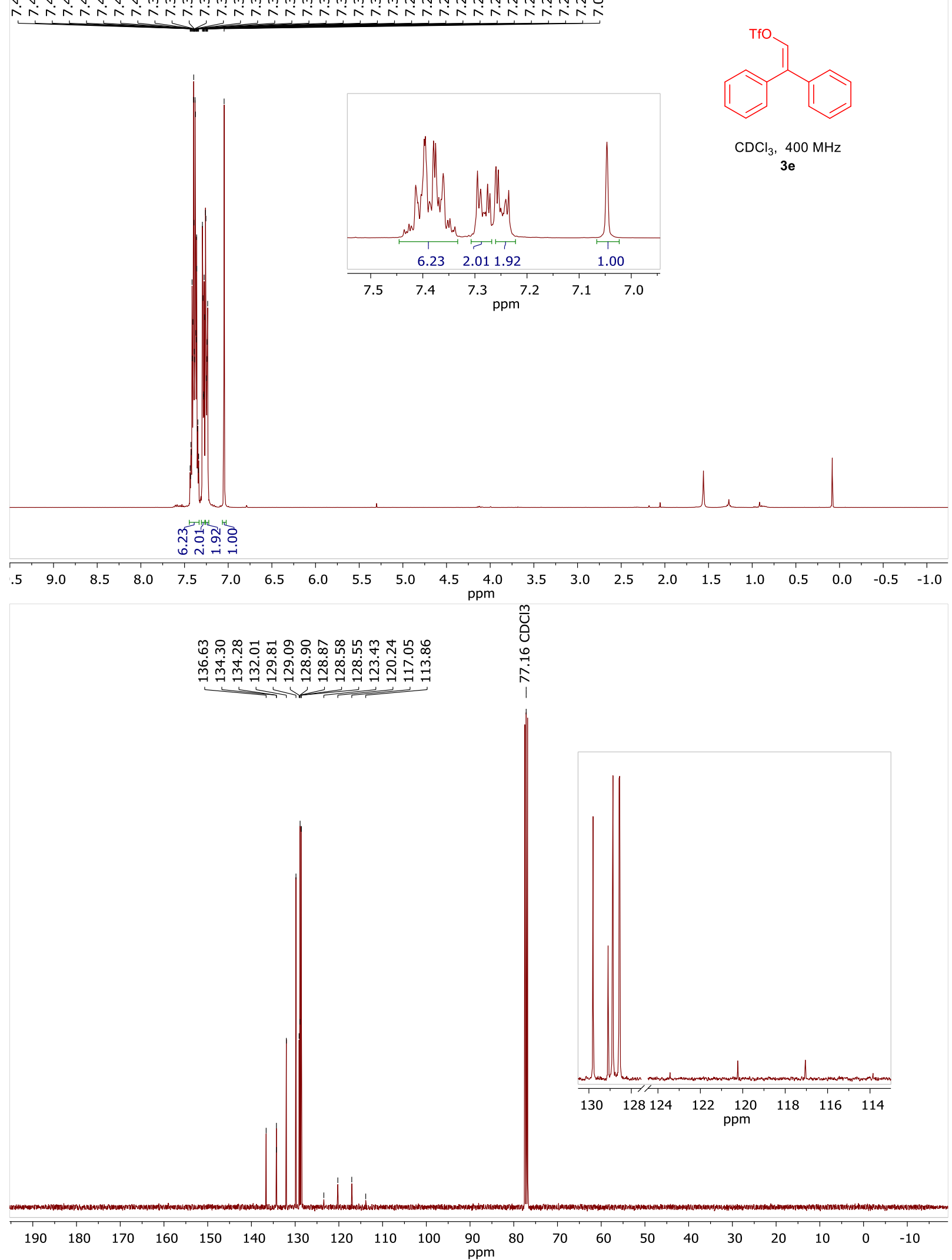


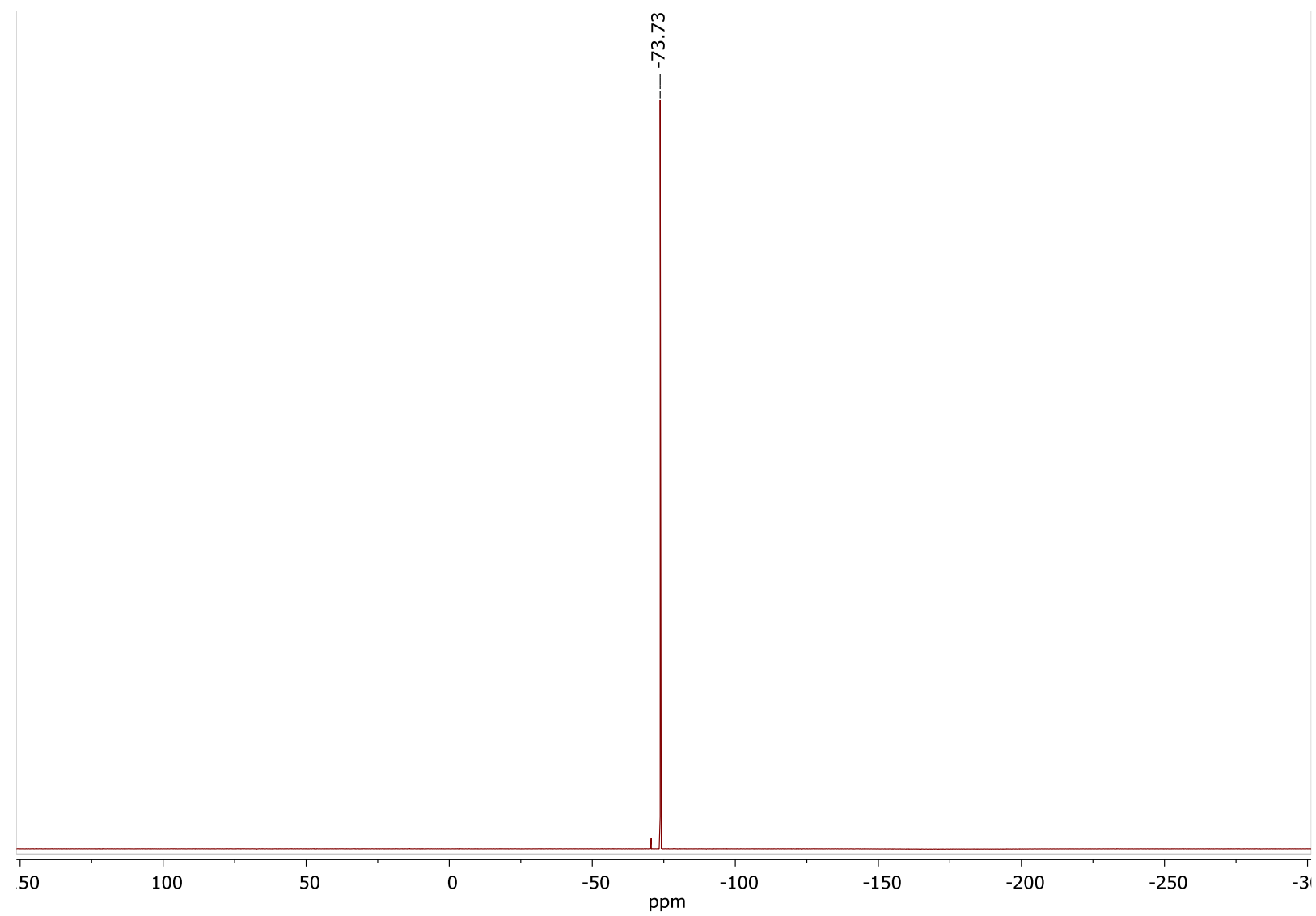

${ }^{1} \mathrm{H},{ }^{13} \mathrm{C}$ and NOESY NMR spectra of compound $4 a$ 要

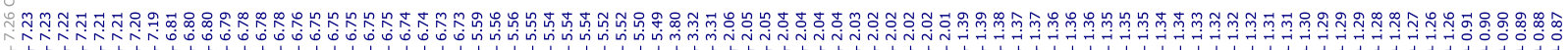

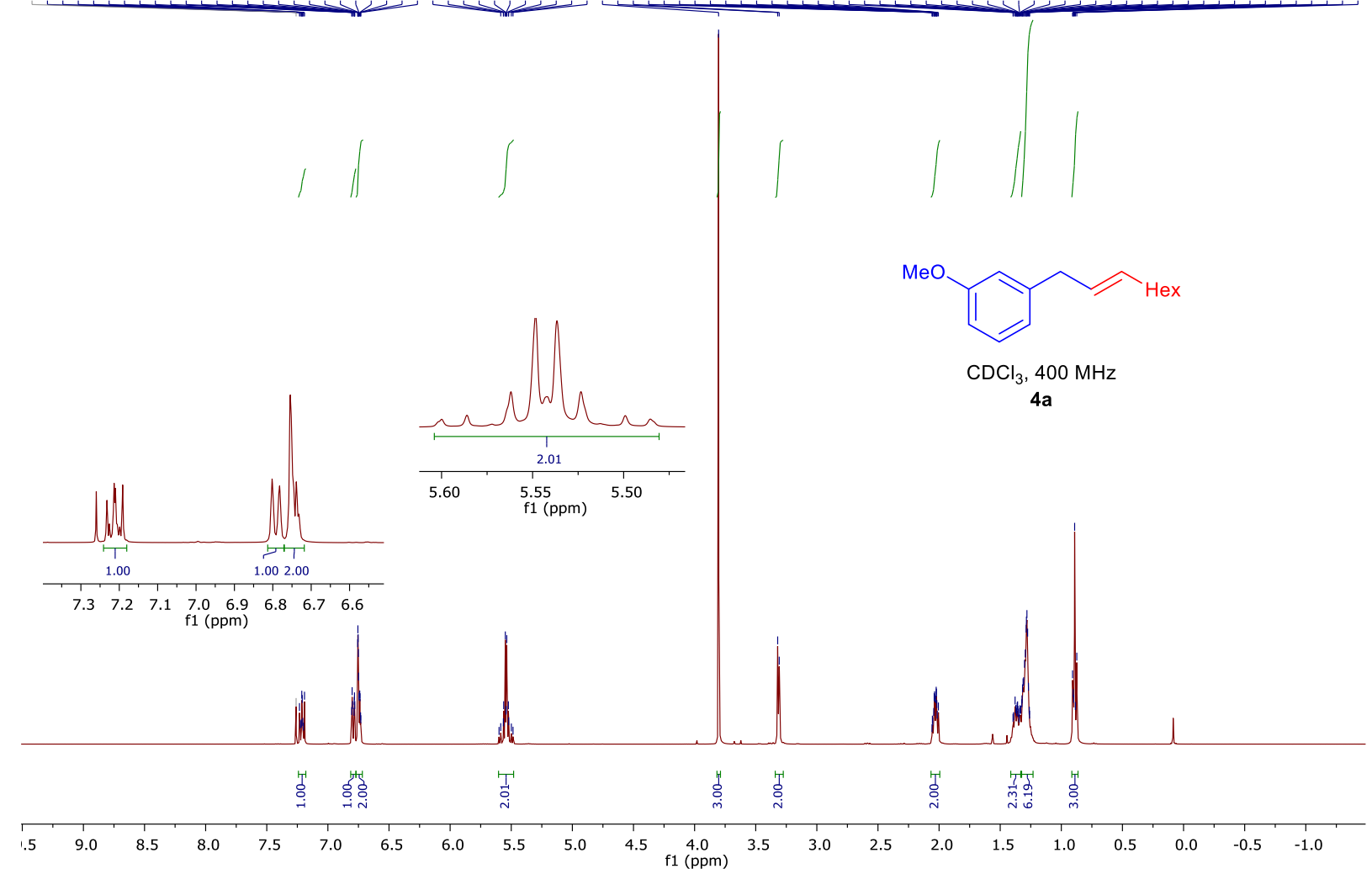




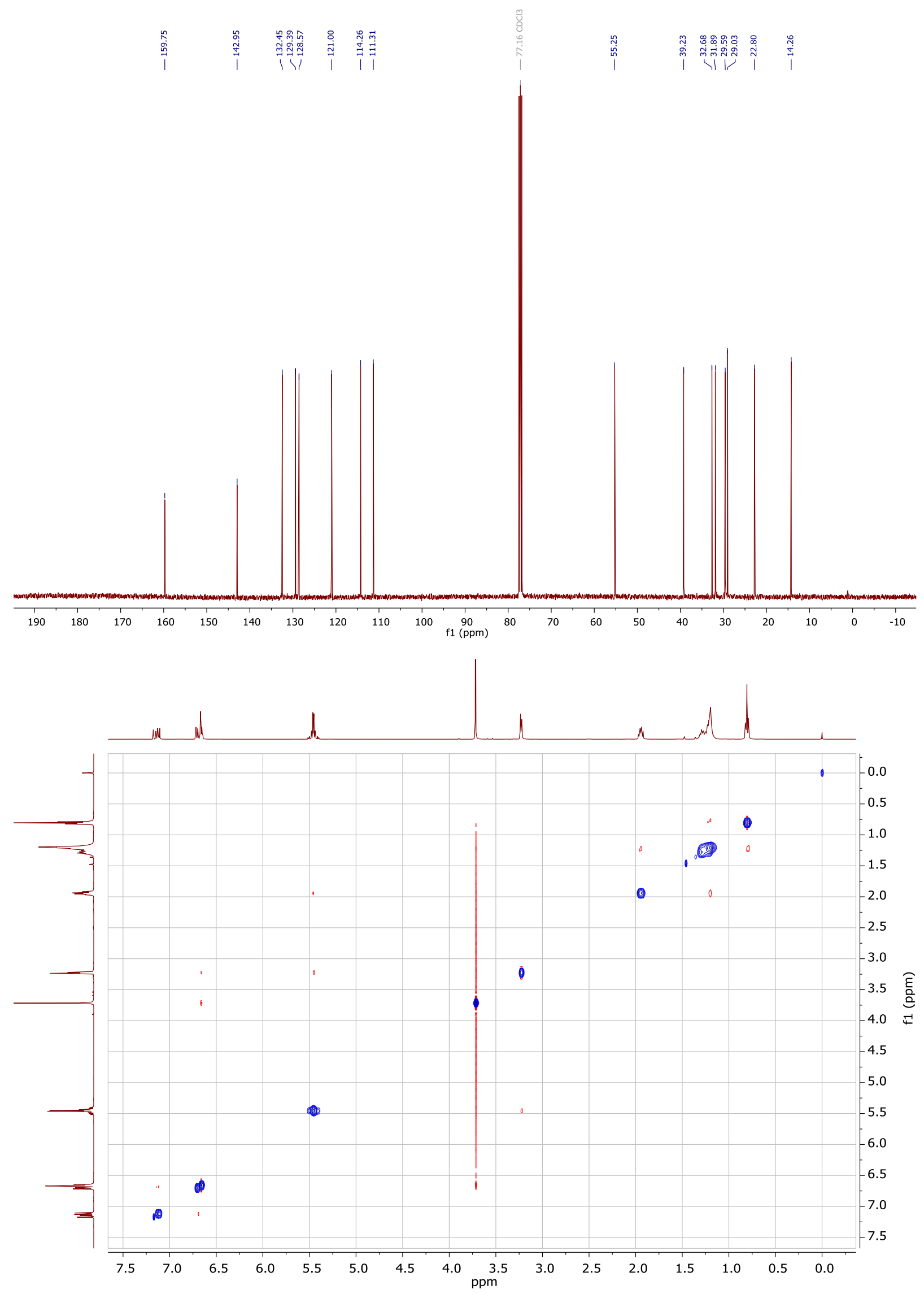


${ }^{1} \mathrm{H}$ and ${ }^{13} \mathrm{C}$ NMR spectra of compound $4 \mathrm{~b}$

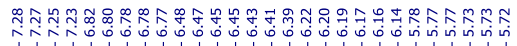

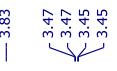
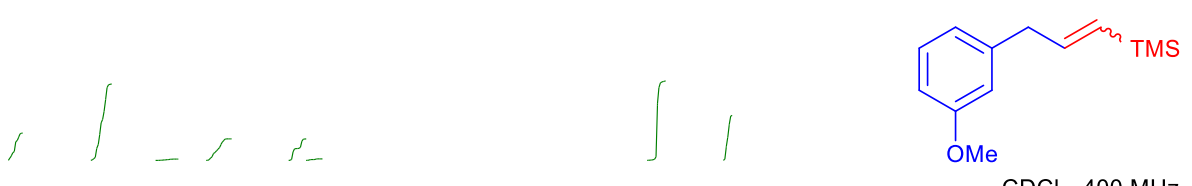

$\mathrm{CDCl}_{3}, 400 \mathrm{MHz}$

$4 b$
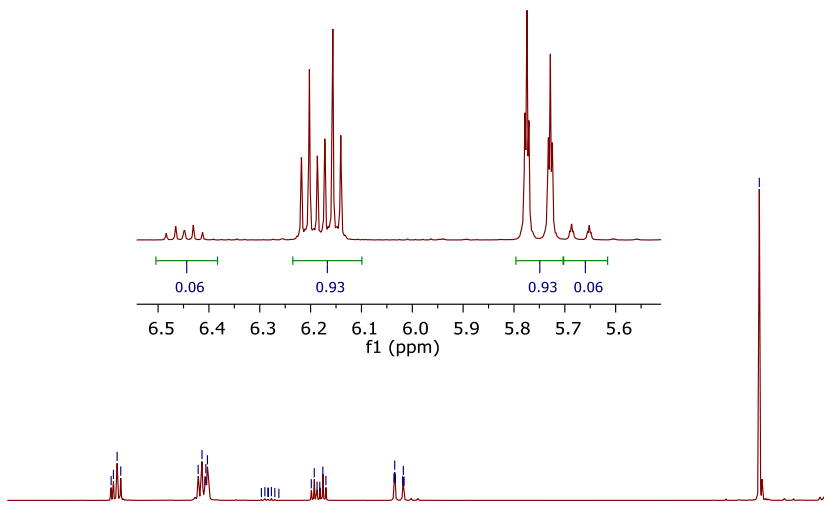

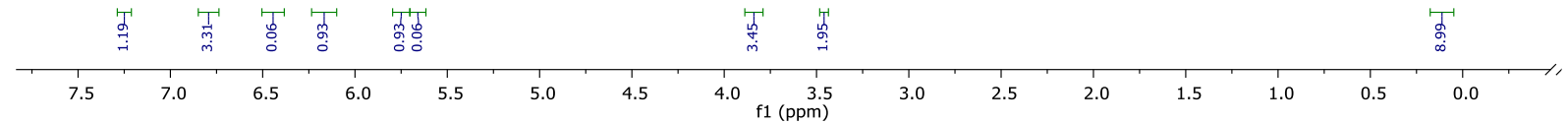

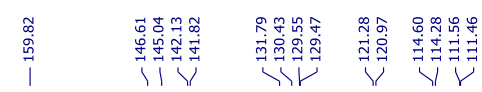

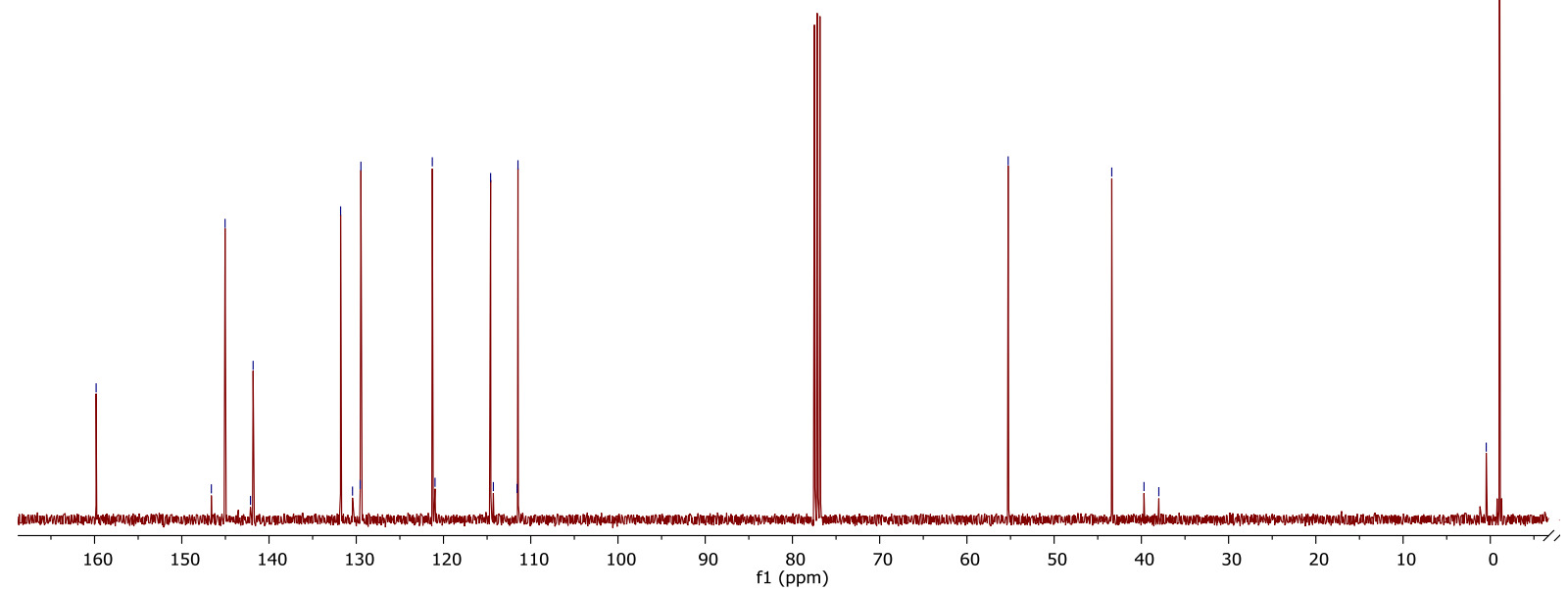


${ }^{1} \mathrm{H}$ and ${ }^{13} \mathrm{C}$ NMR spectra of compound 4c

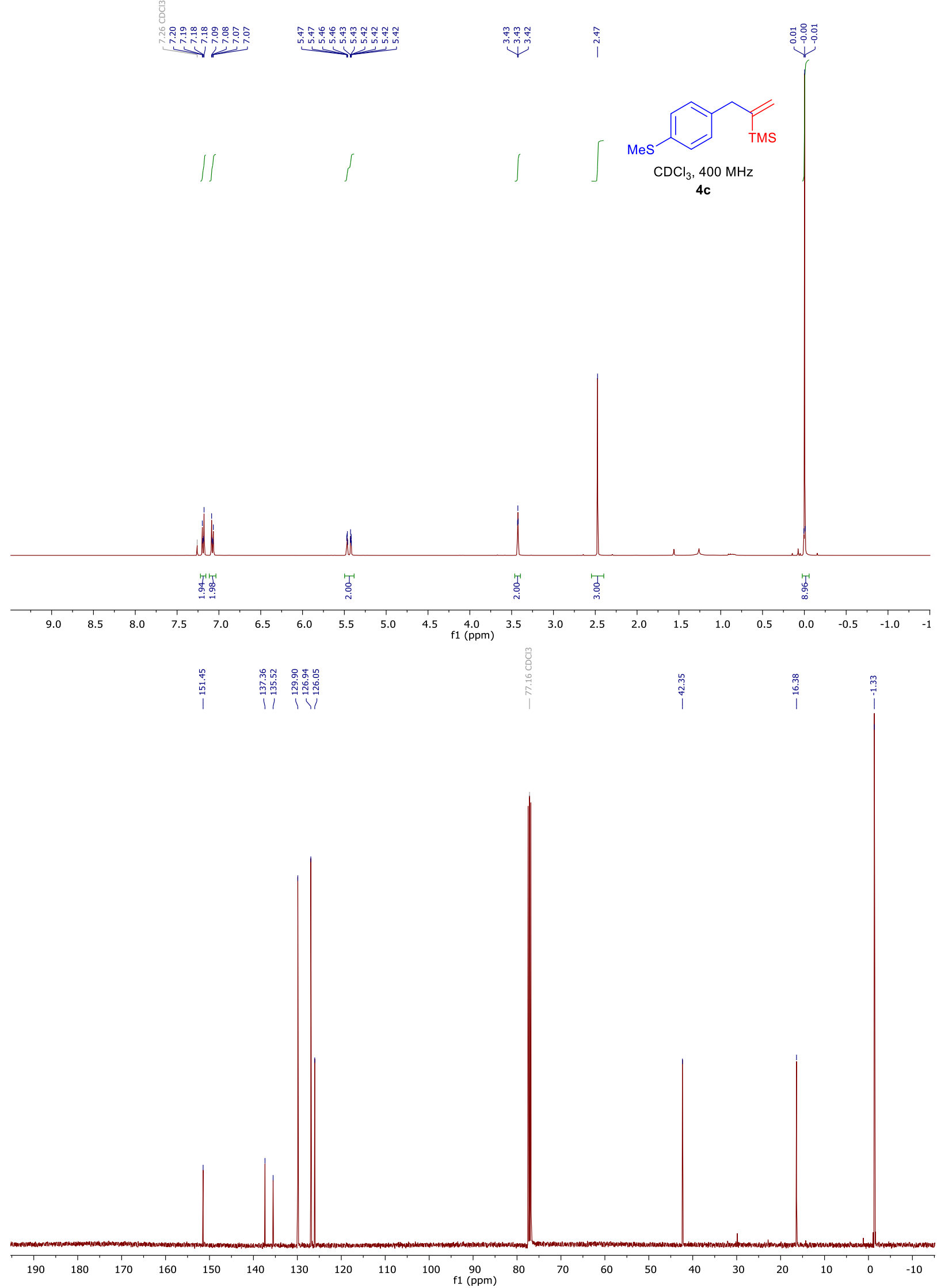


${ }^{1} \mathrm{H},{ }^{13} \mathrm{C}$ and HSQC NMR spectra of compound $\mathbf{4 d}$

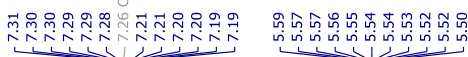

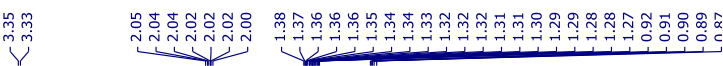
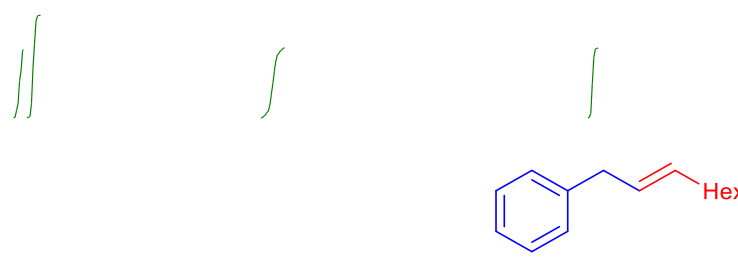

$\mathrm{CDCl}_{3}, 400 \mathrm{MHz}$

4d
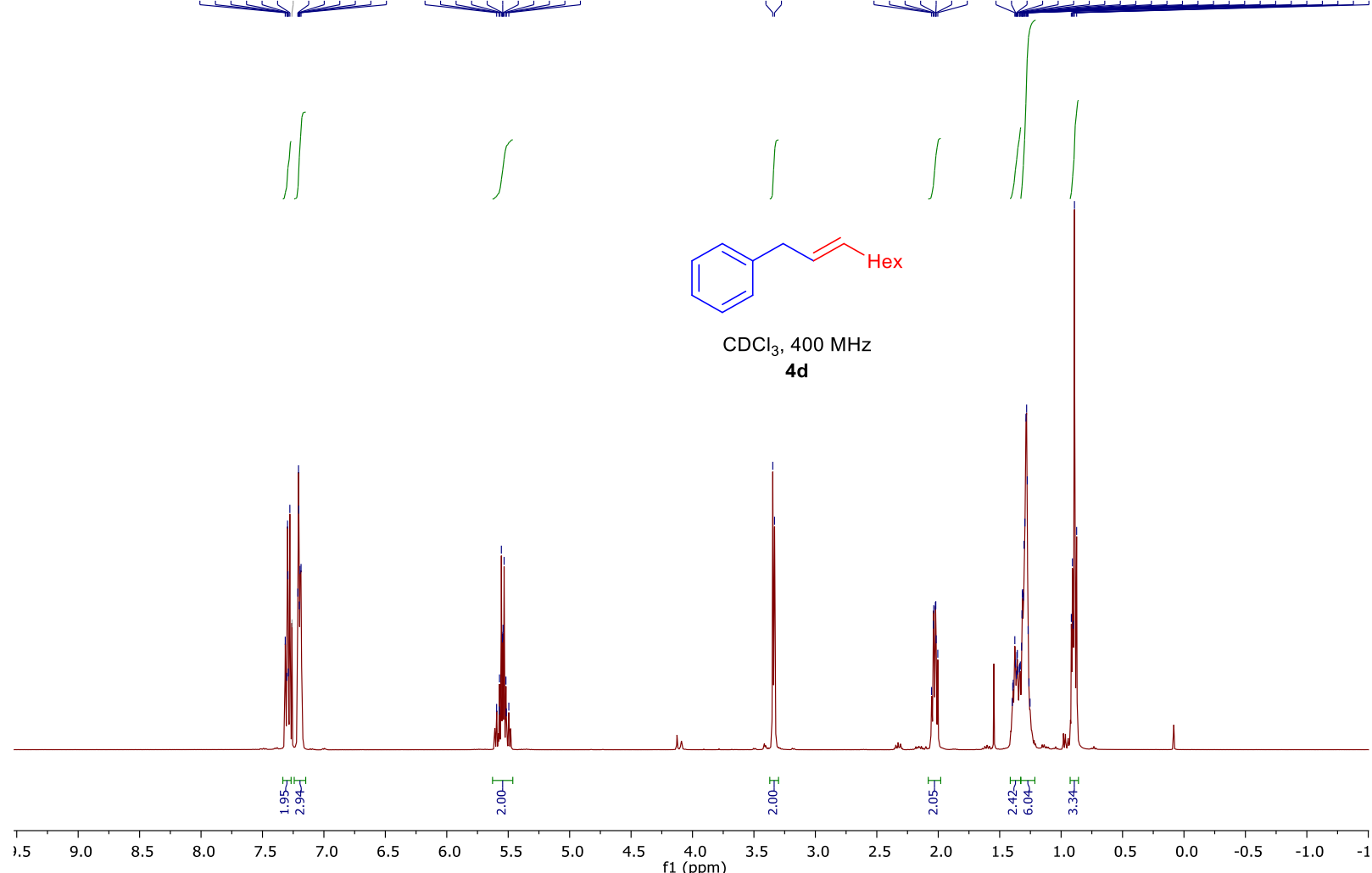

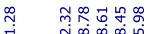

華

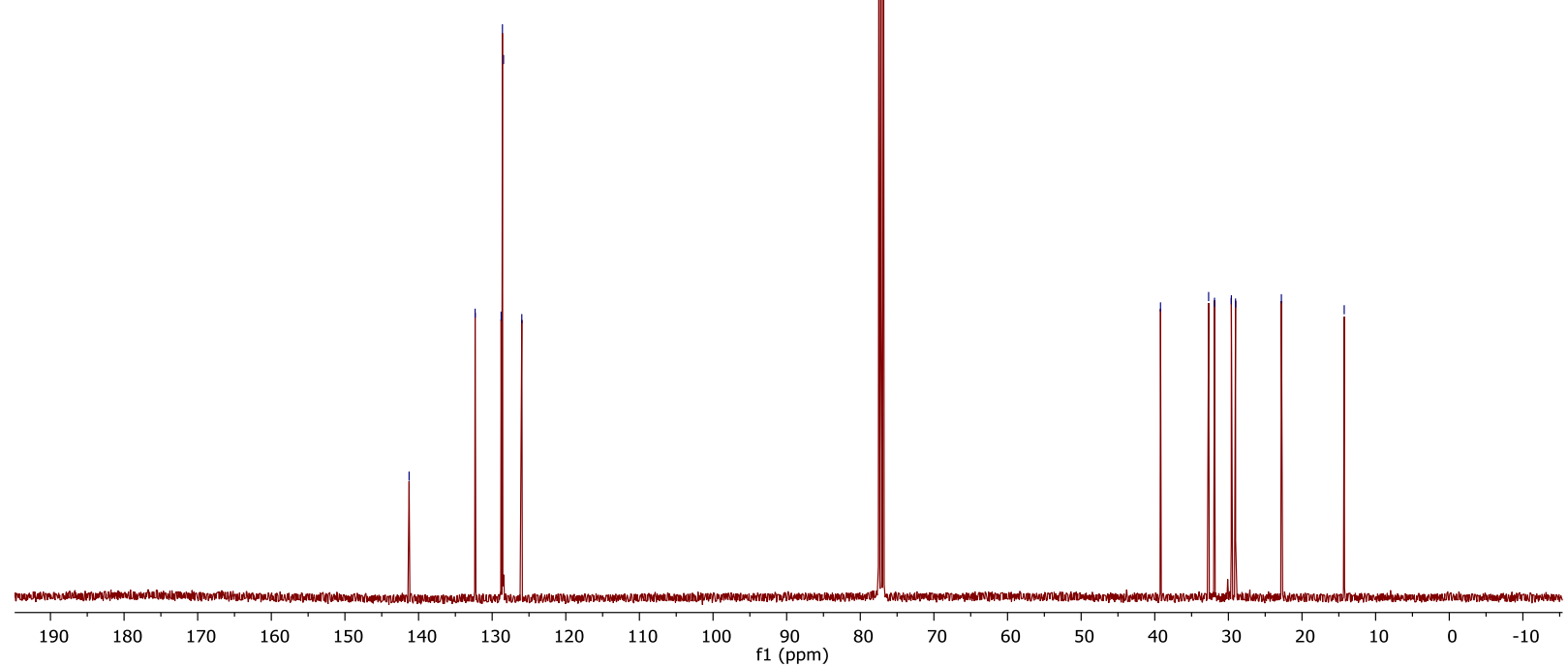




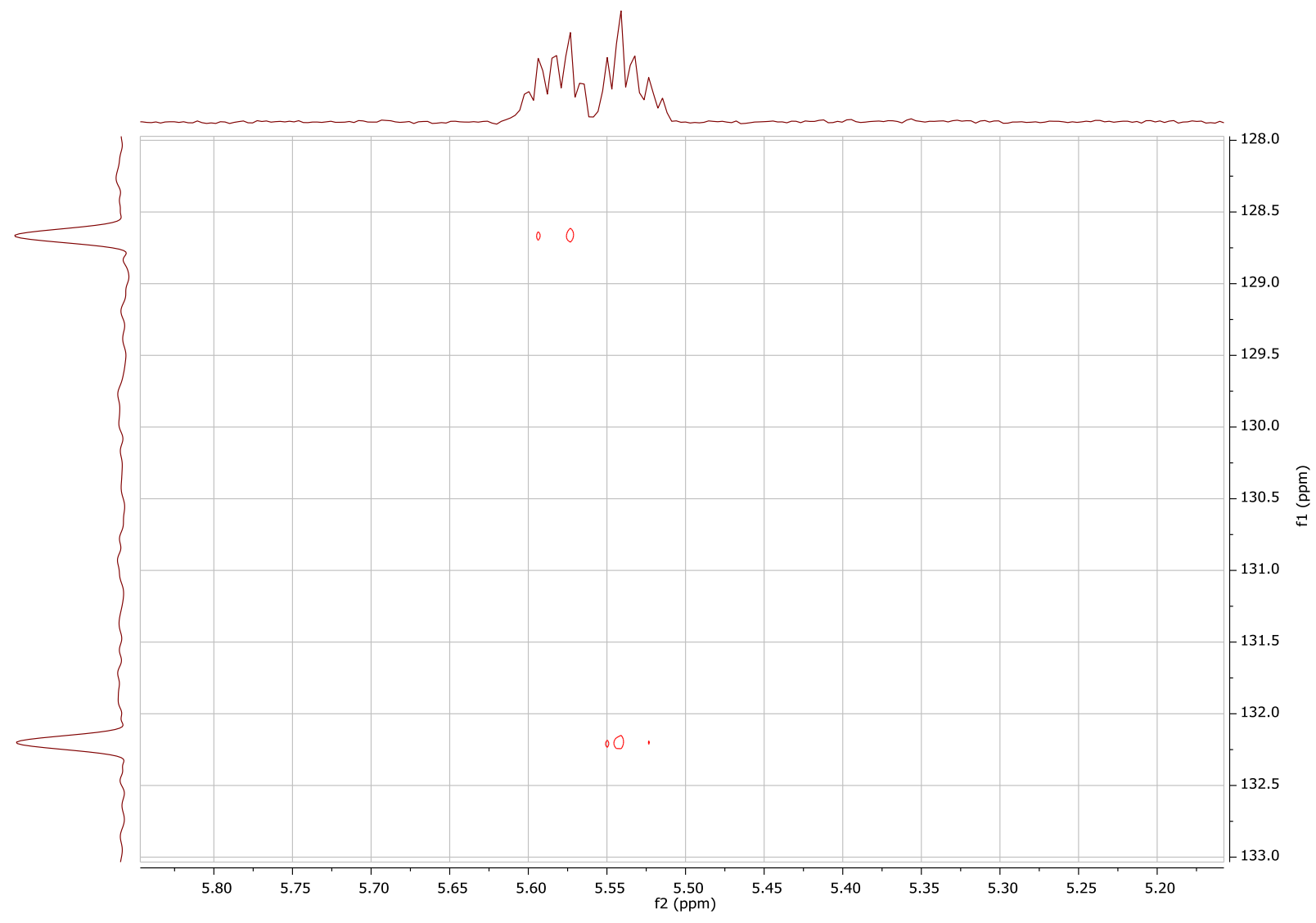

${ }^{1} \mathrm{H}$ and ${ }^{13} \mathrm{C}$ NMR spectra of compound $4 e$

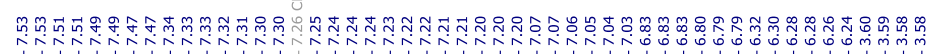

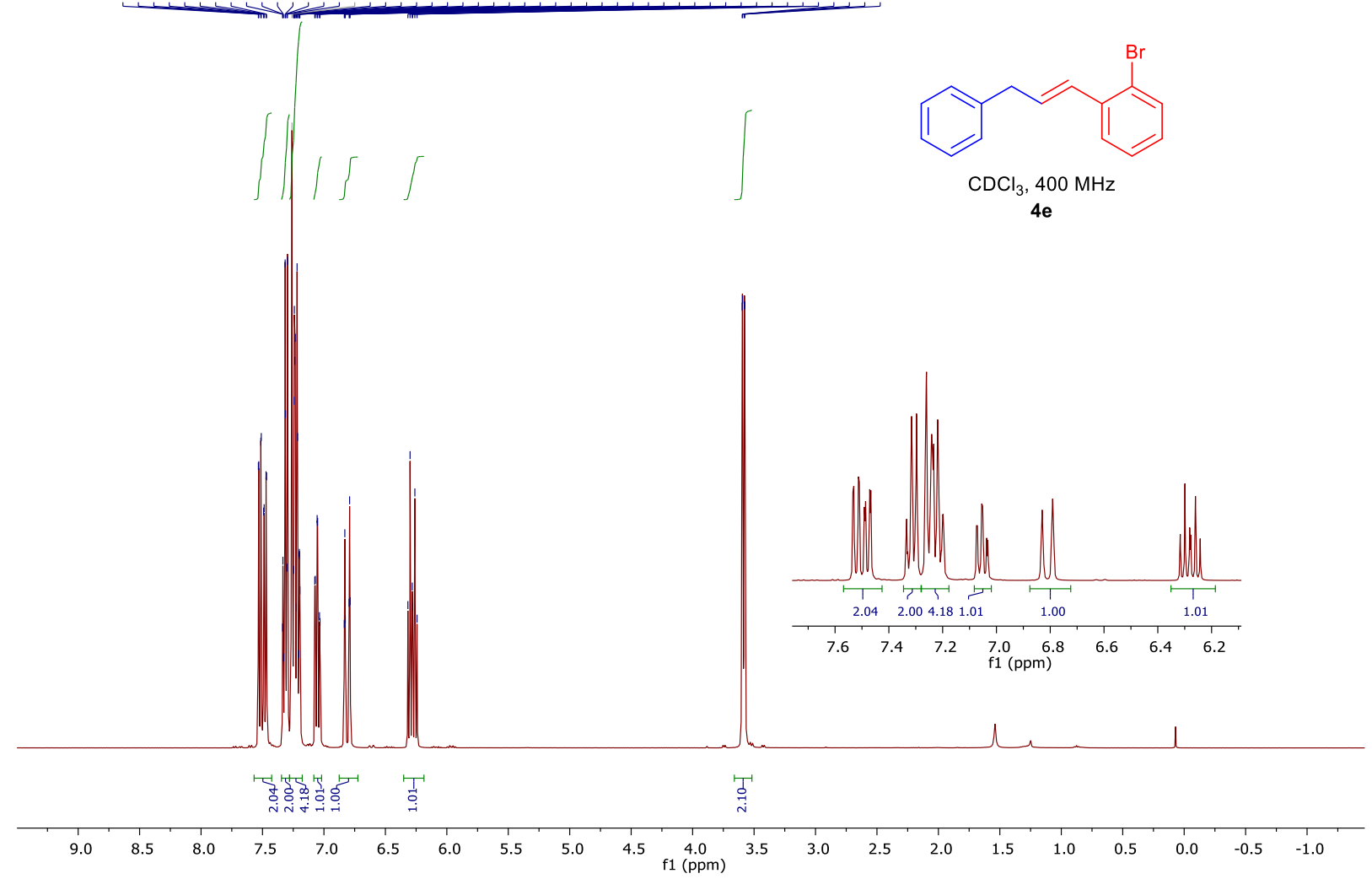




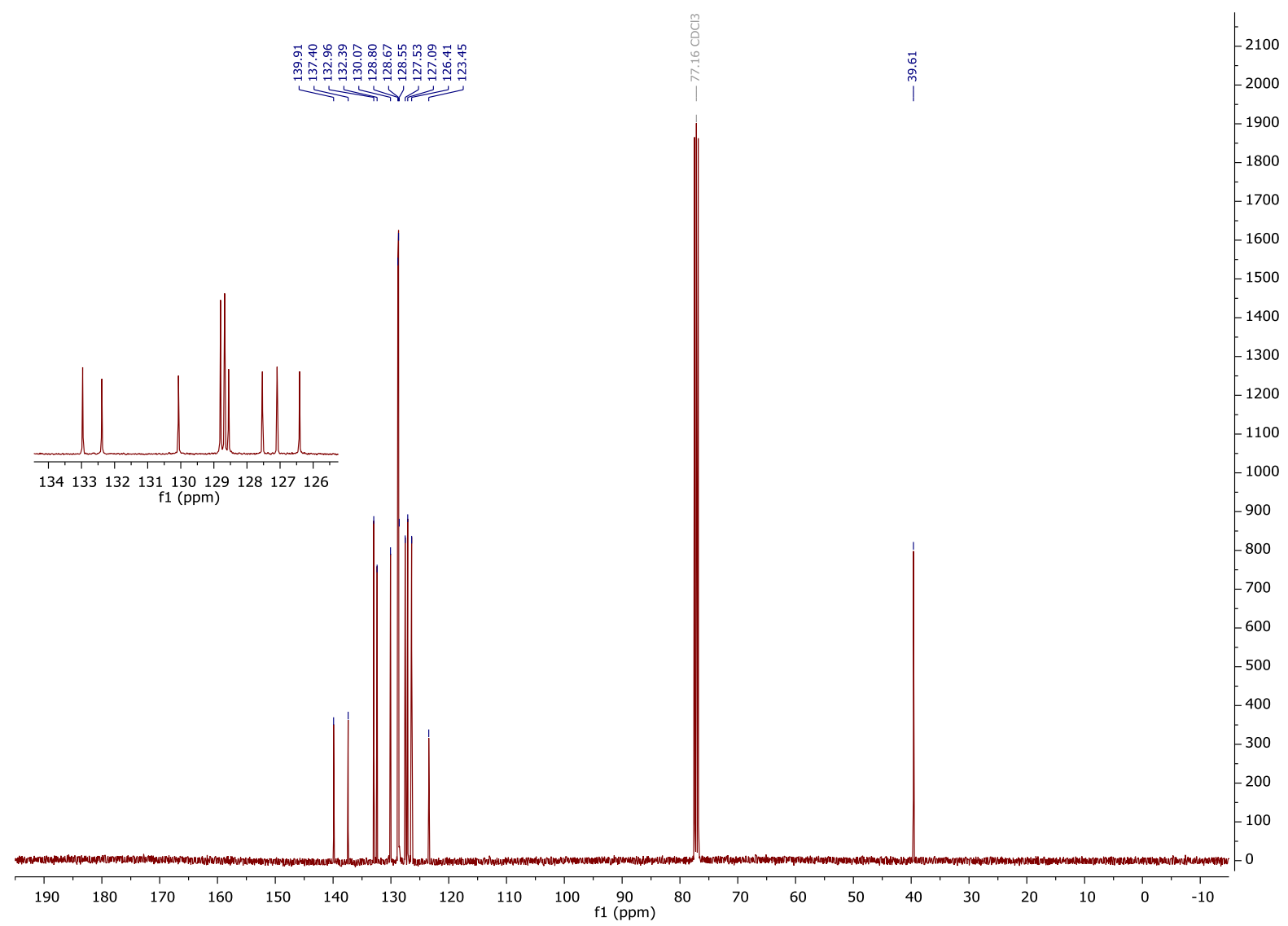

${ }^{1} \mathrm{H}$ and ${ }^{13} \mathrm{C}$ NMR spectra of compound $\mathbf{4 f}$

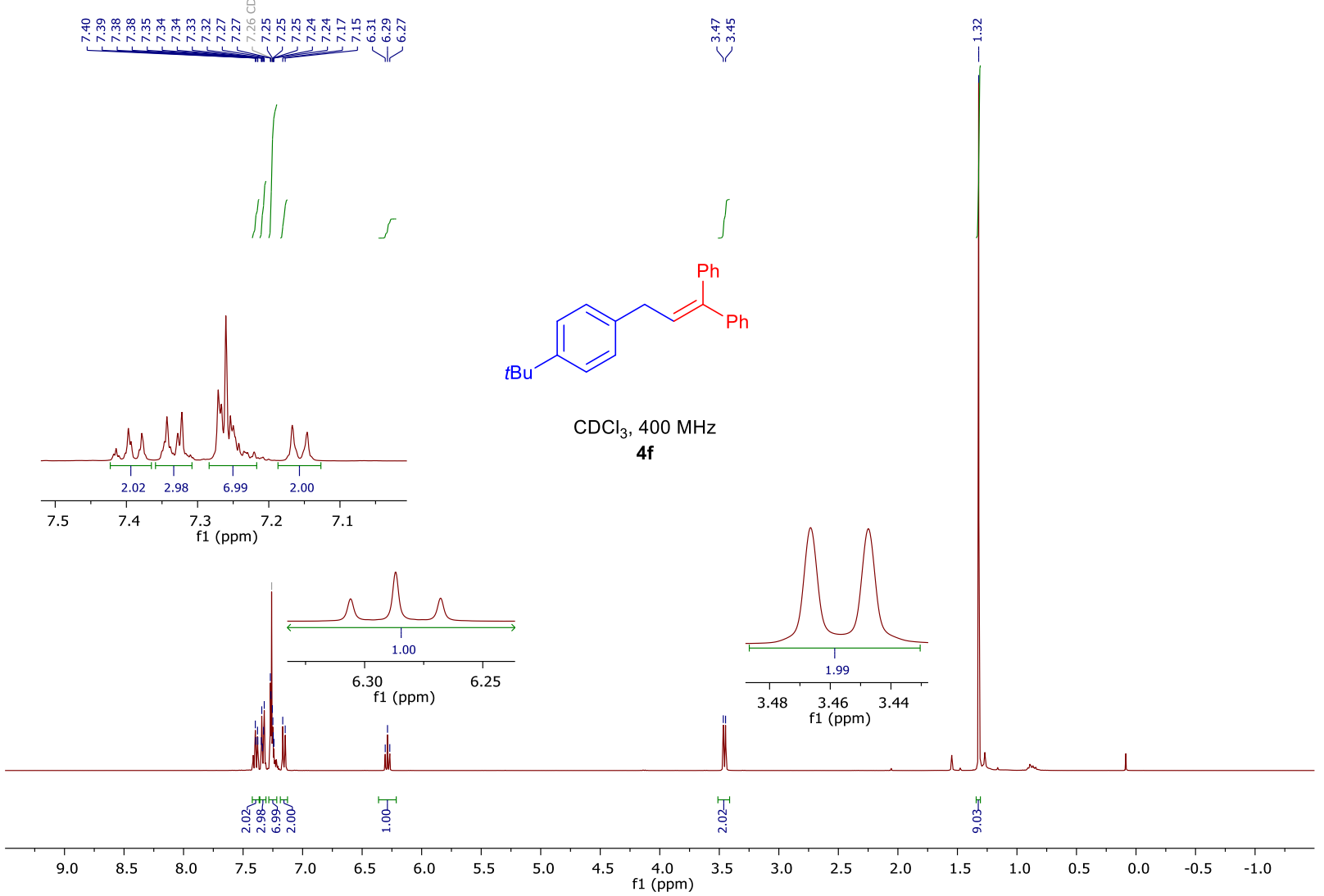




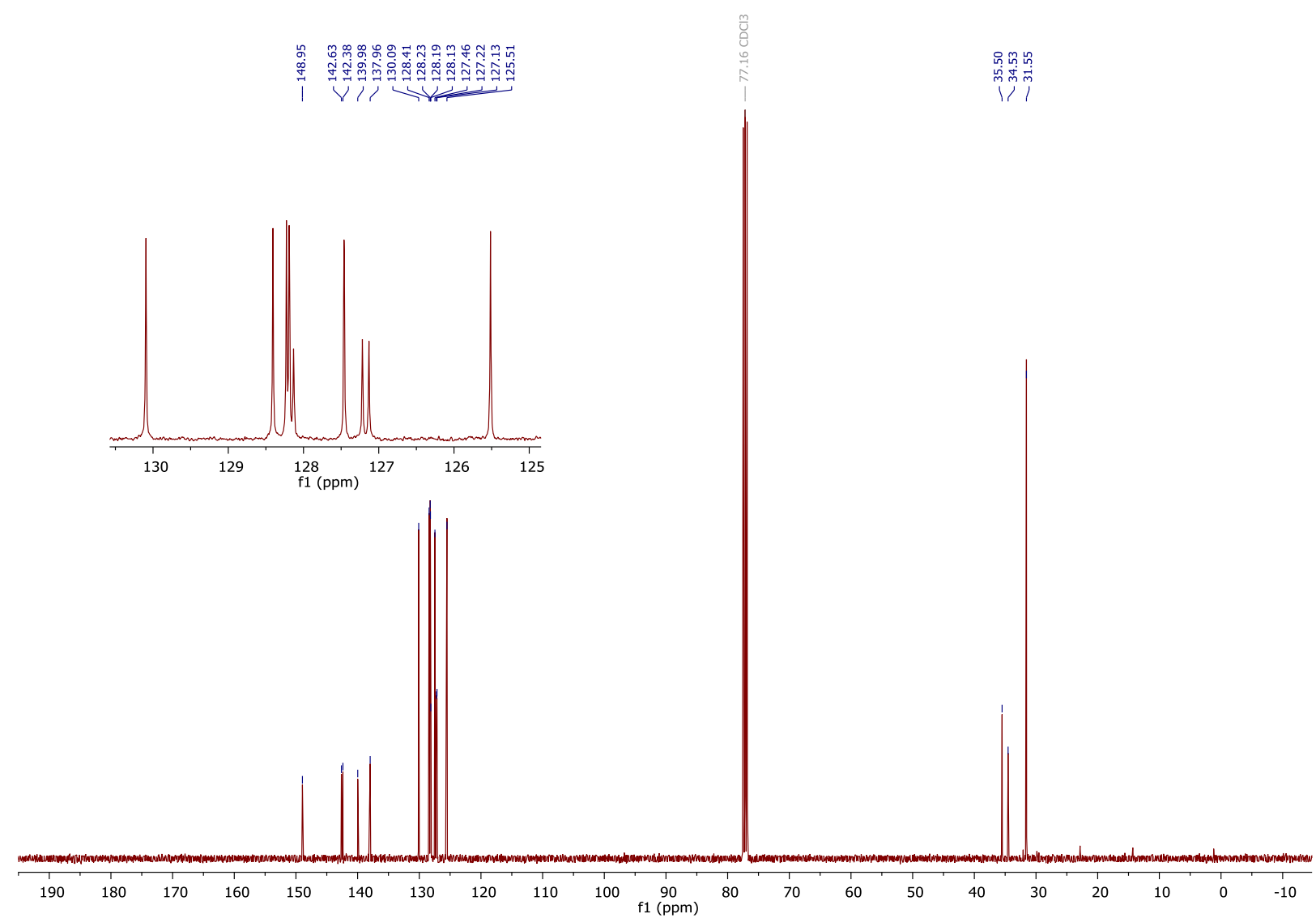

${ }^{1} \mathrm{H},{ }^{13} \mathrm{C}$ and NOESY NMR spectra of compound $\mathbf{4 g}$

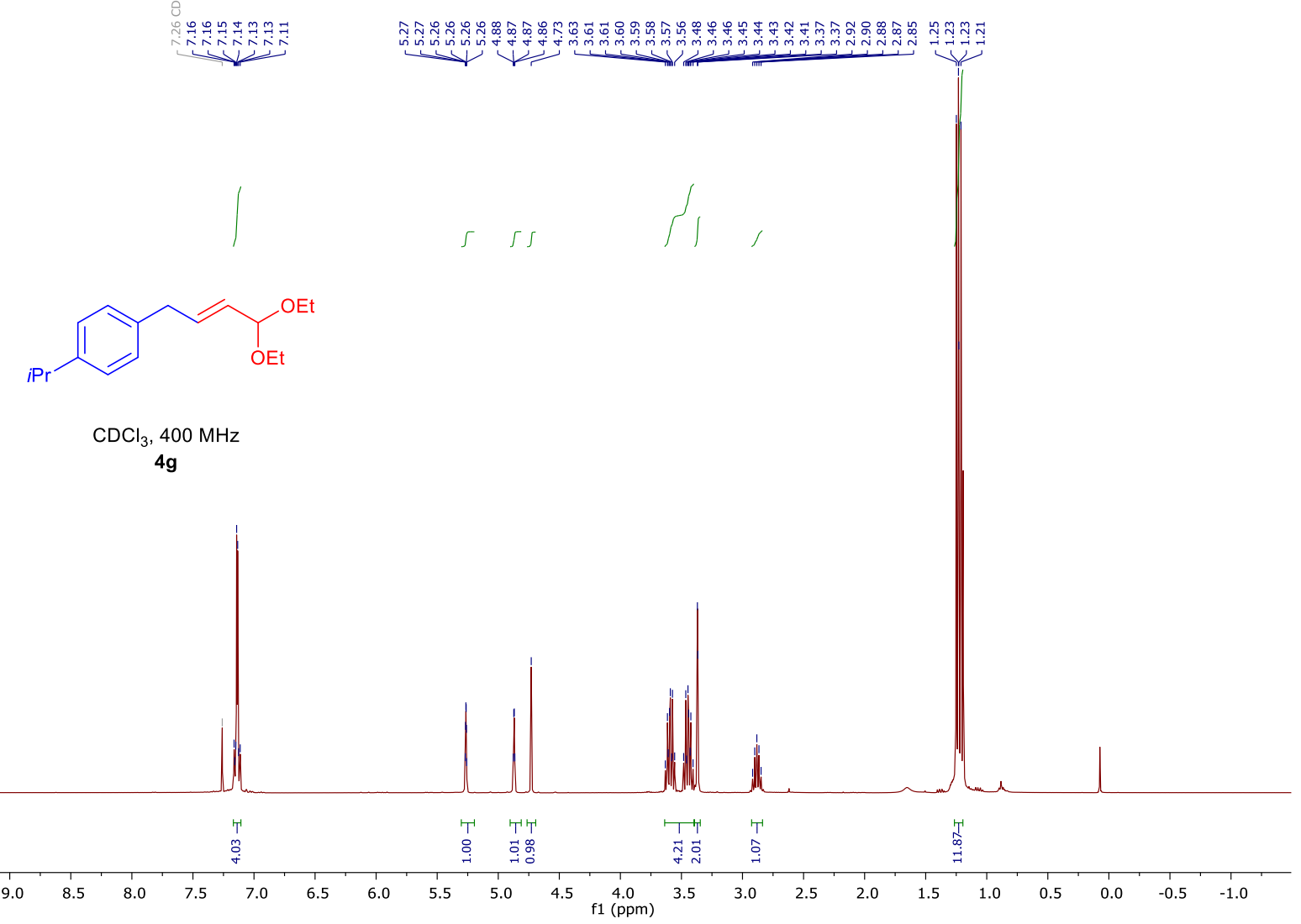




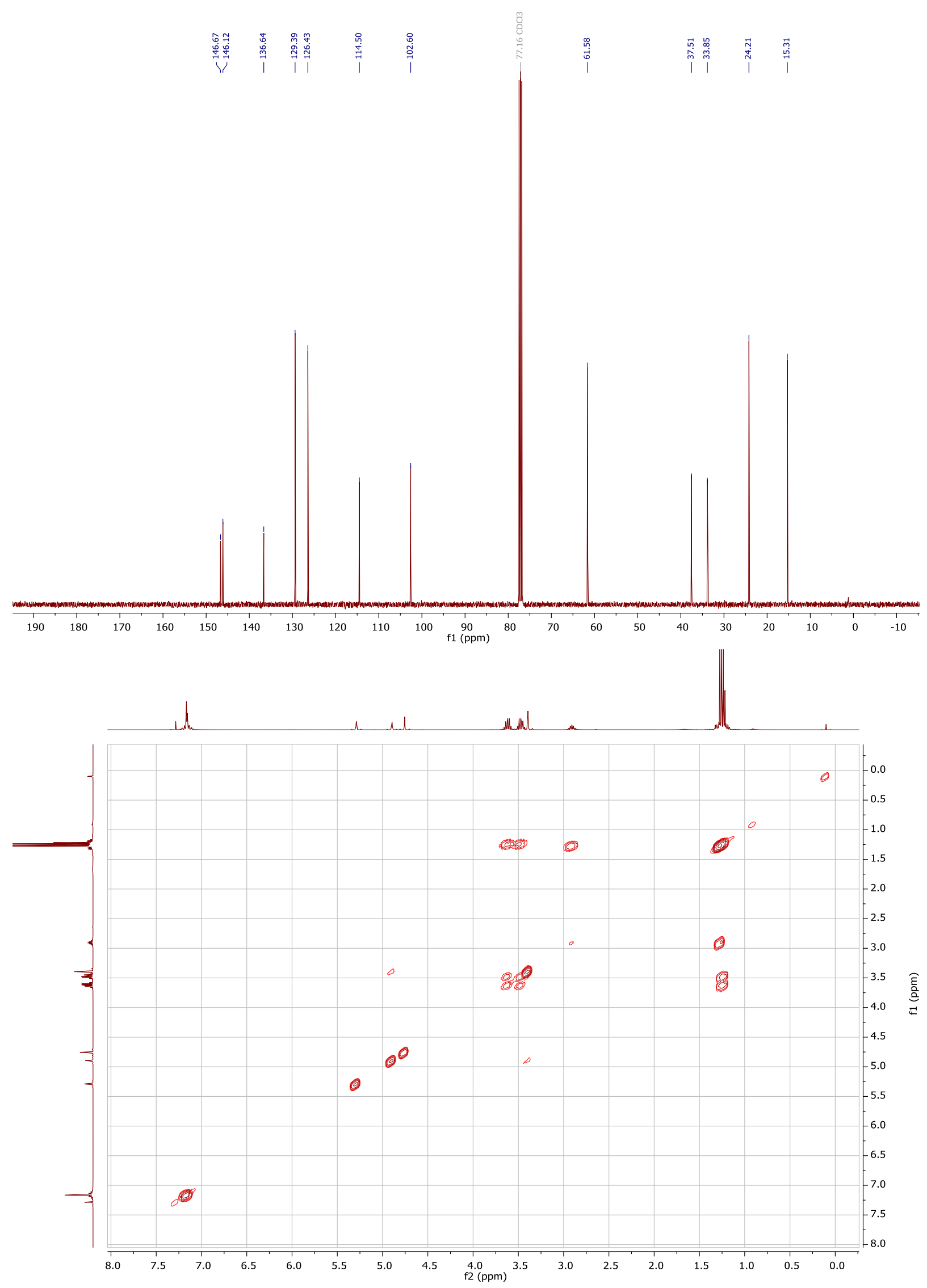


${ }^{1} \mathrm{H}$ and ${ }^{13} \mathrm{C}$ NMR spectra of compound $\mathbf{4 h}$
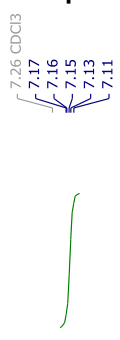

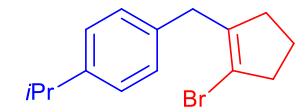

$\mathrm{CDCl}_{3}, 400 \mathrm{MHz}$ $4 \mathrm{~h}$
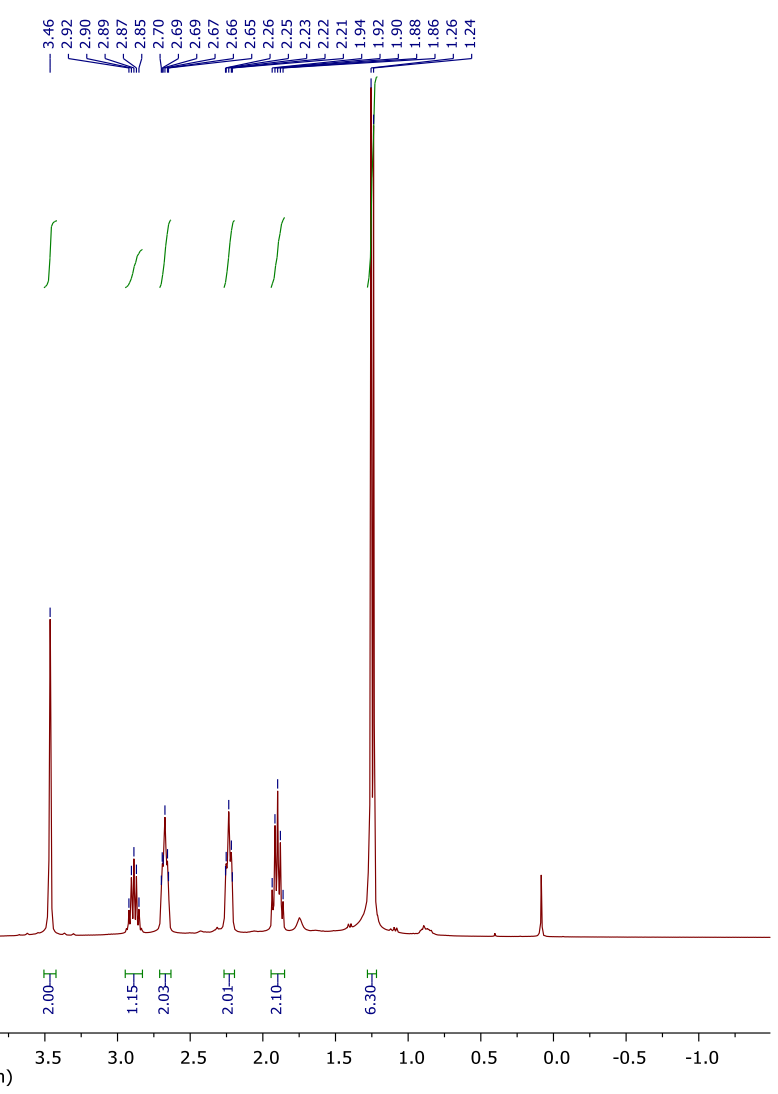

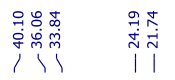

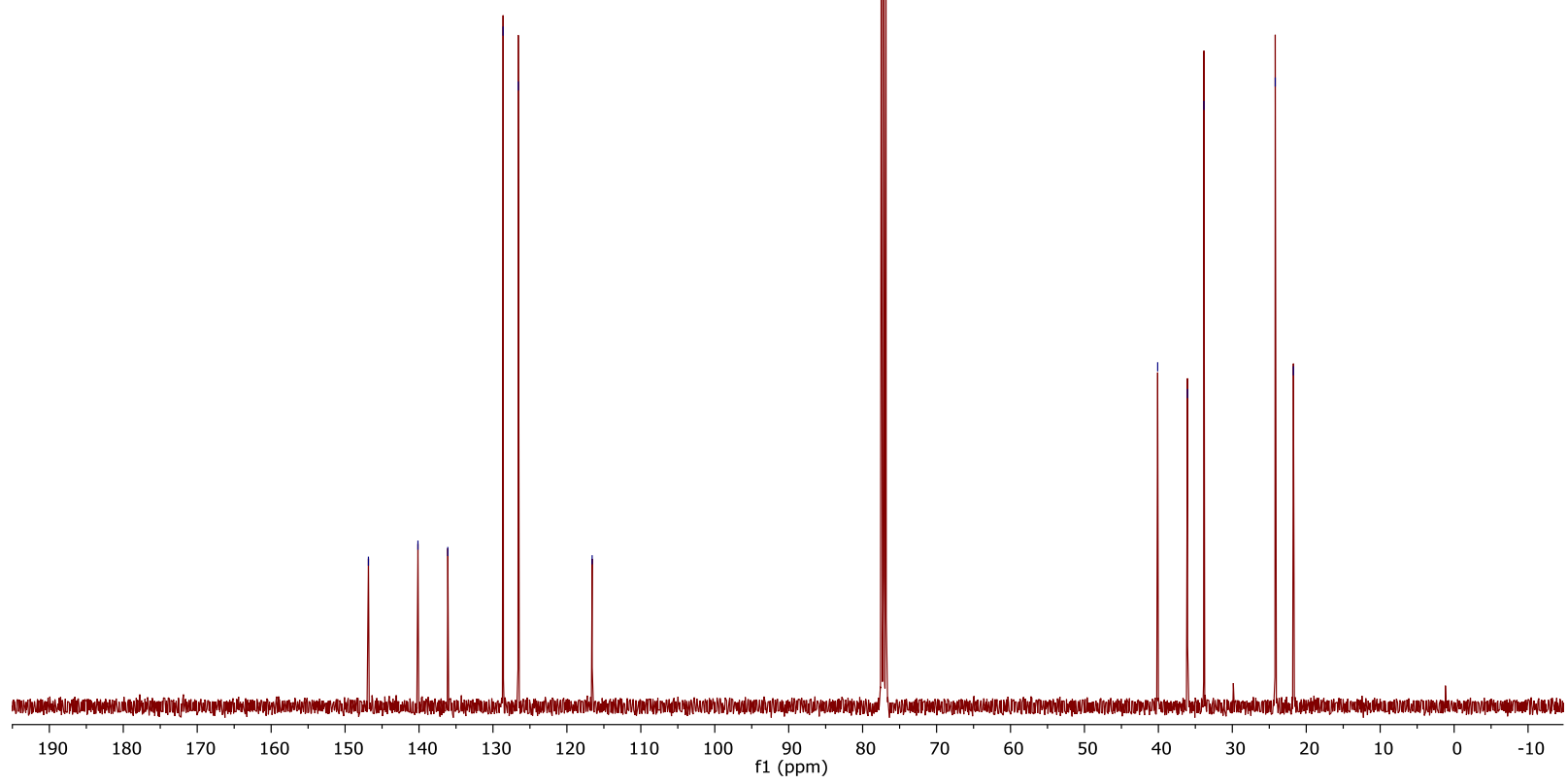

SI-39 
${ }^{1} \mathrm{H},{ }^{13} \mathrm{C}$ and ${ }^{19} \mathrm{~F}$ NMR spectra of compound $4 \mathrm{i}$

耑

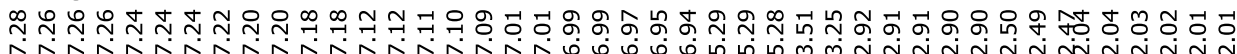

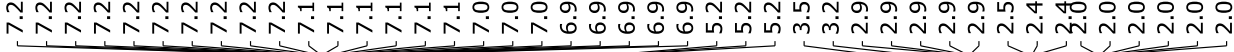

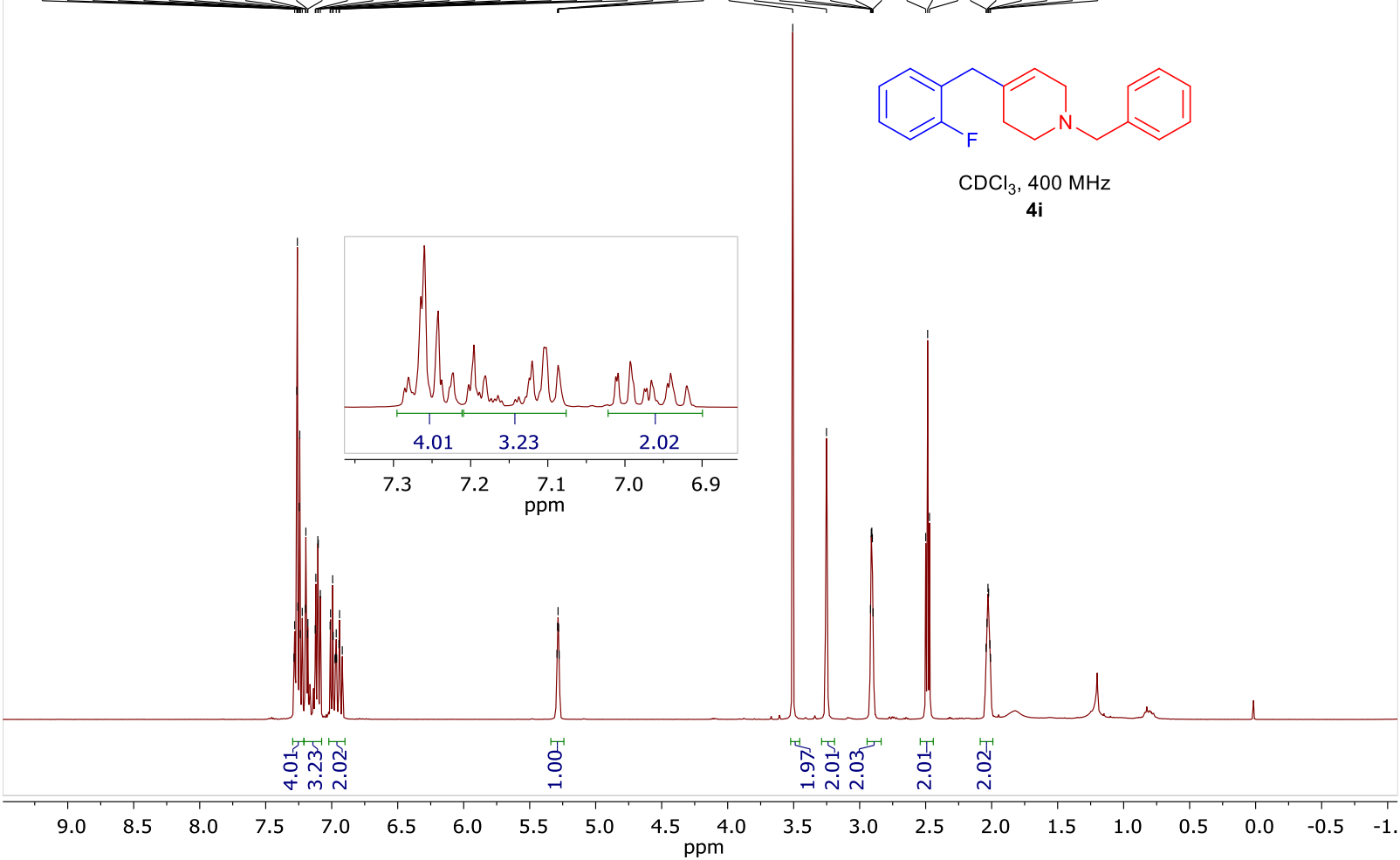

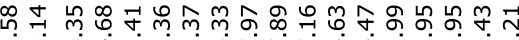

它完

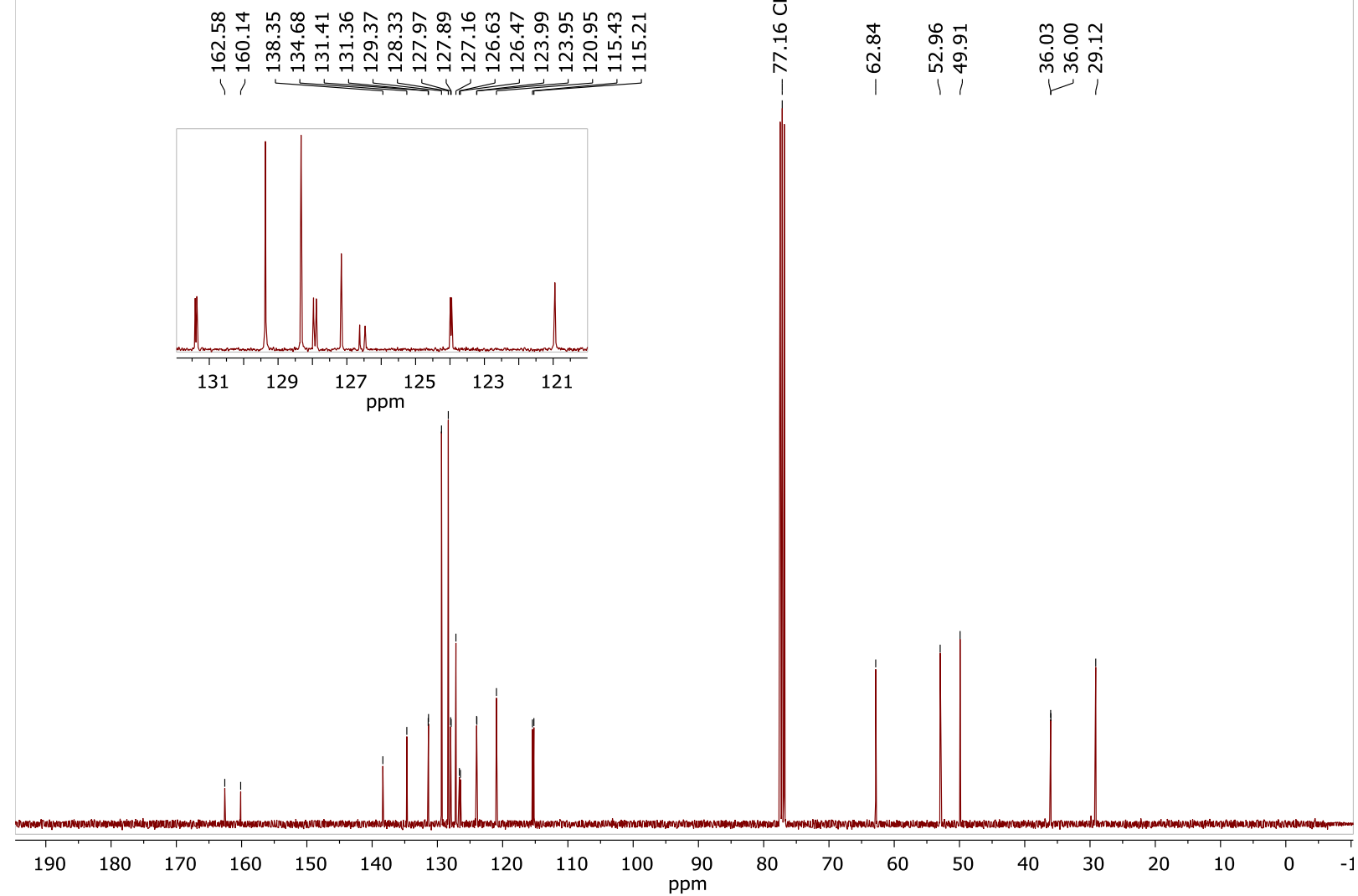




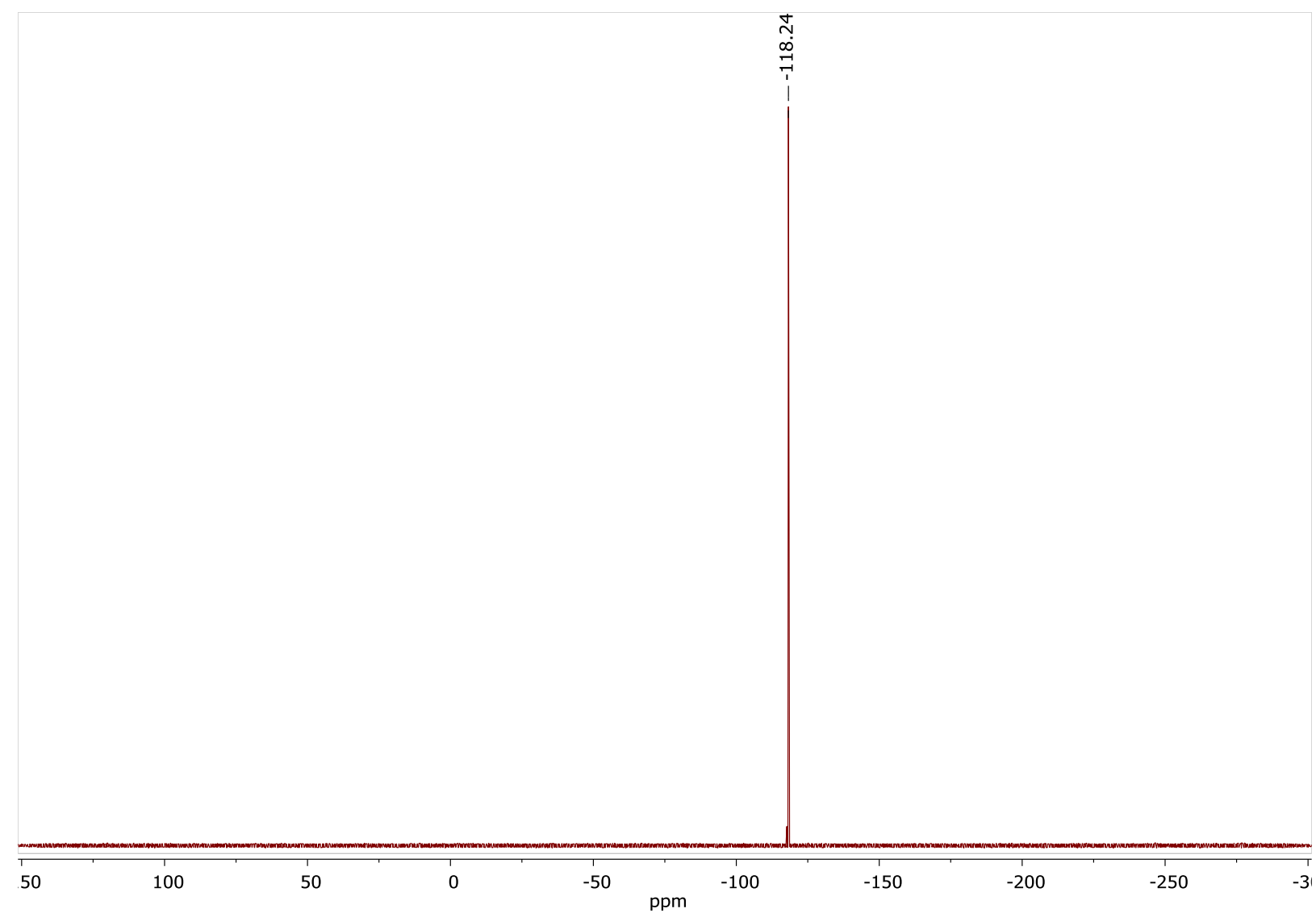

${ }^{1} \mathrm{H},{ }^{13} \mathrm{C},{ }^{19} \mathrm{~F}$ and HSQC NMR spectra of compound $\mathbf{4 j}$

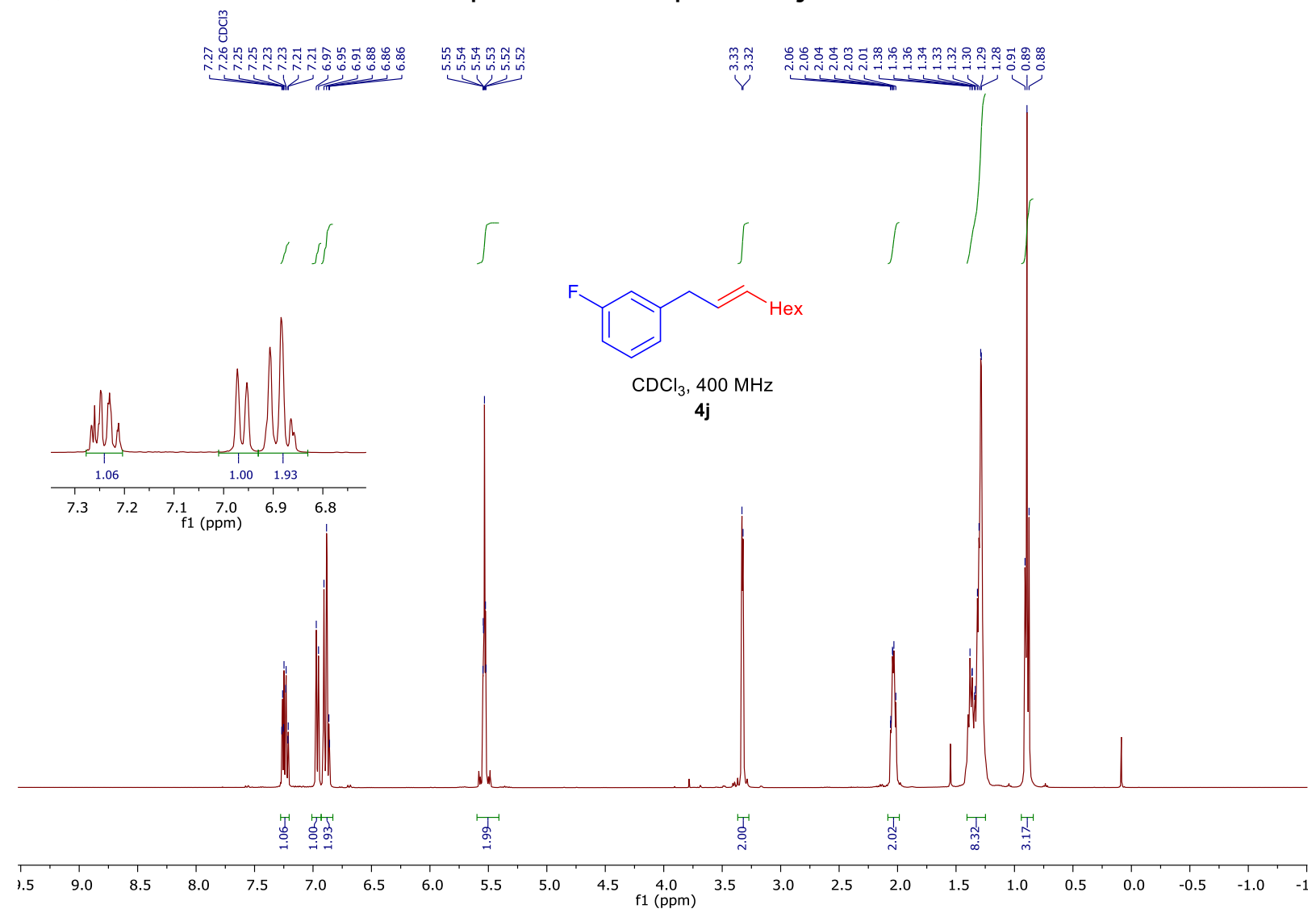




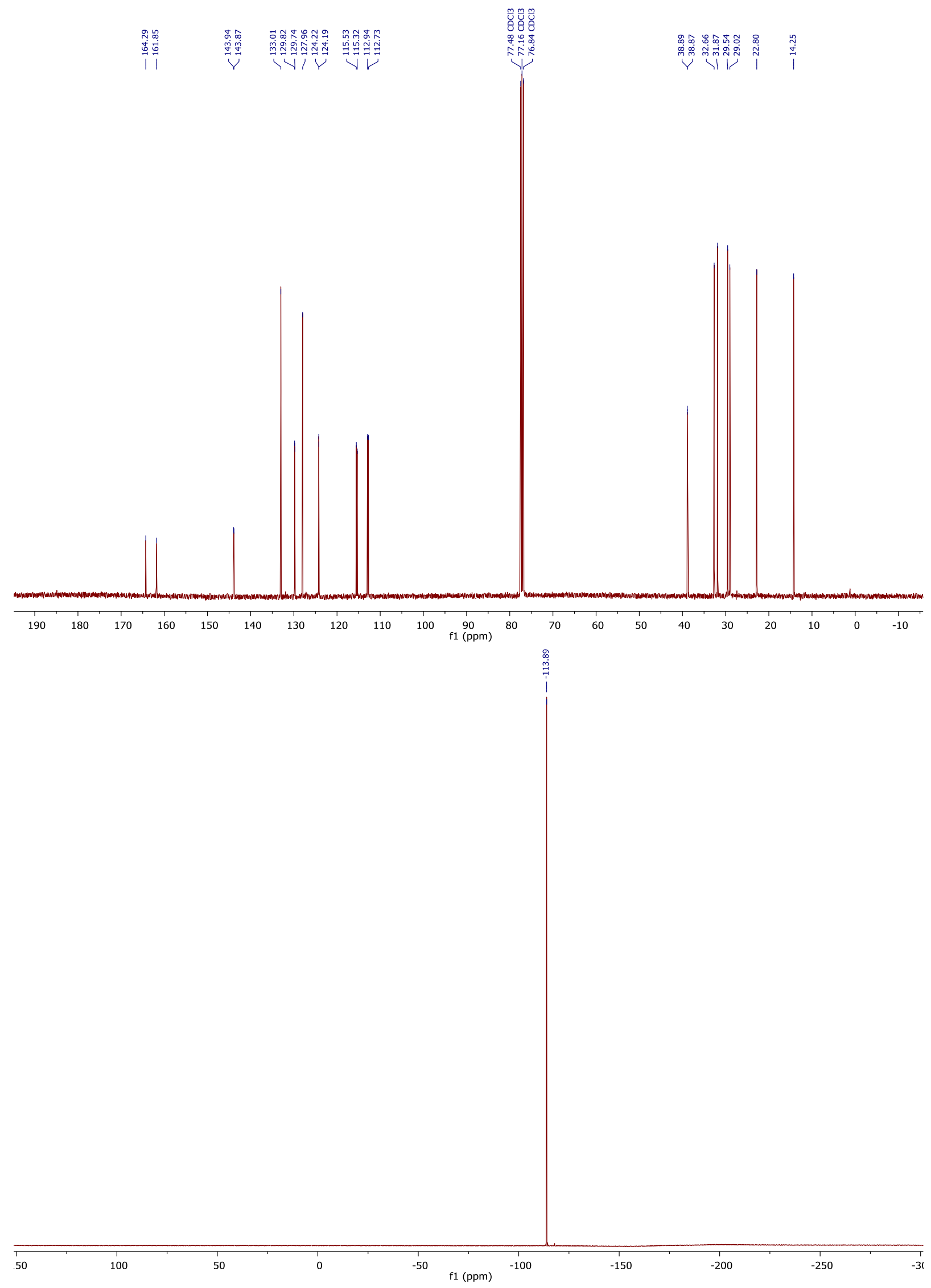




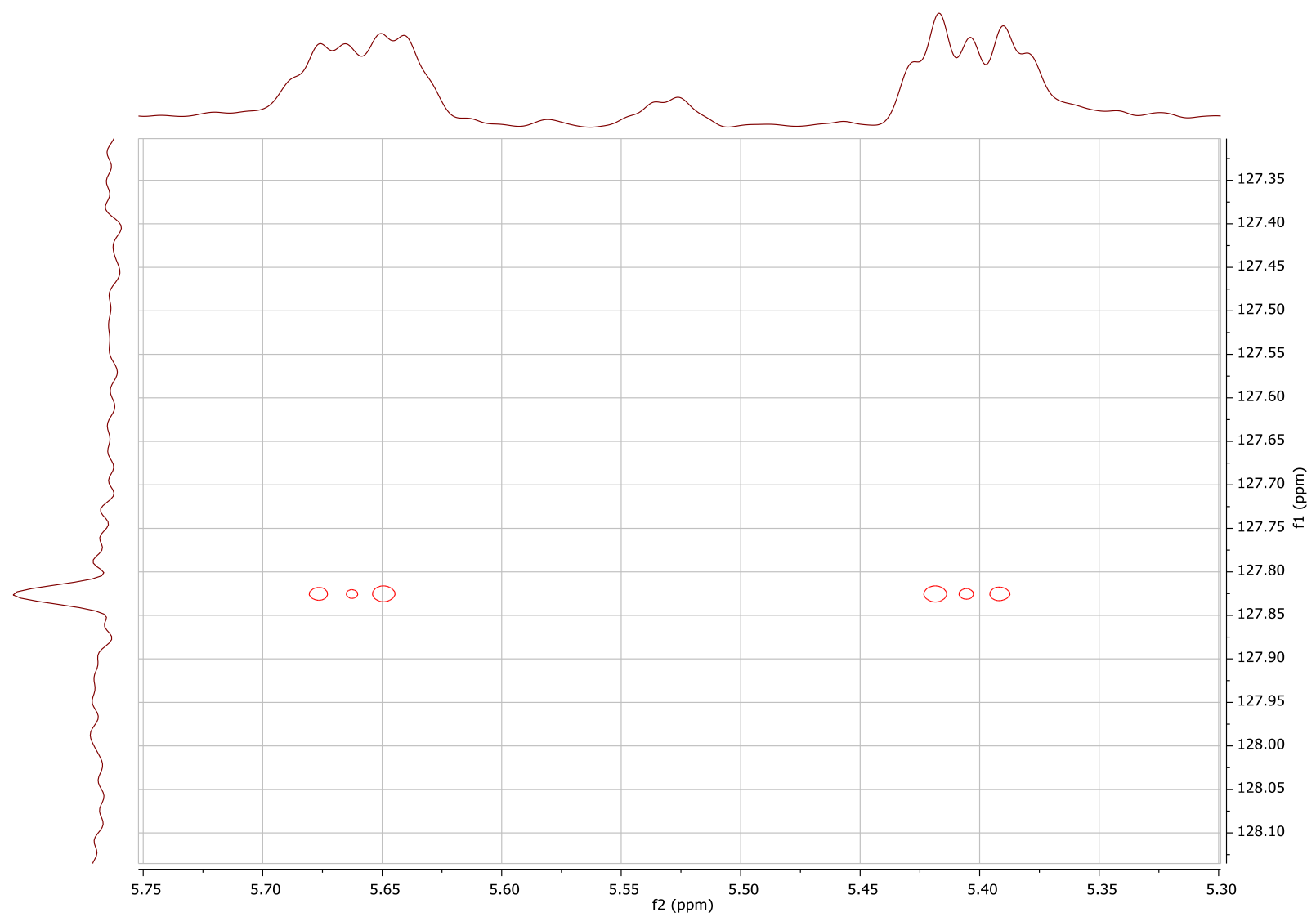

${ }^{1} \mathrm{H},{ }^{13} \mathrm{C}$ and ${ }^{19} \mathrm{~F}$ NMR spectra of compound $\mathbf{4 k}$

岂

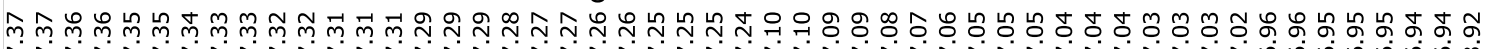

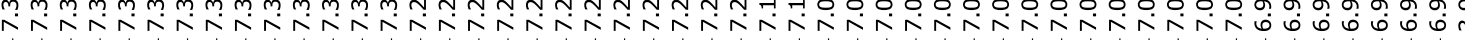

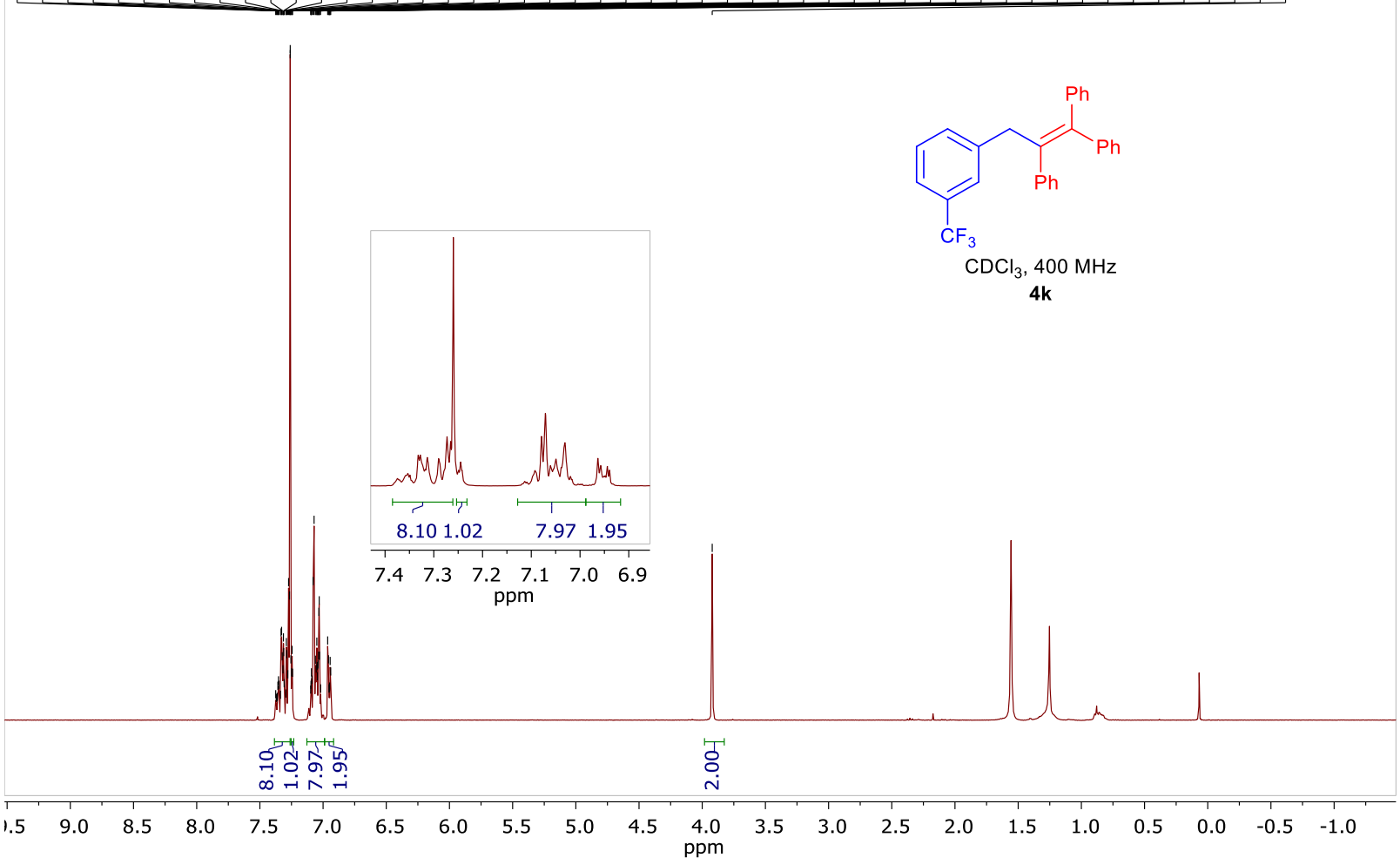



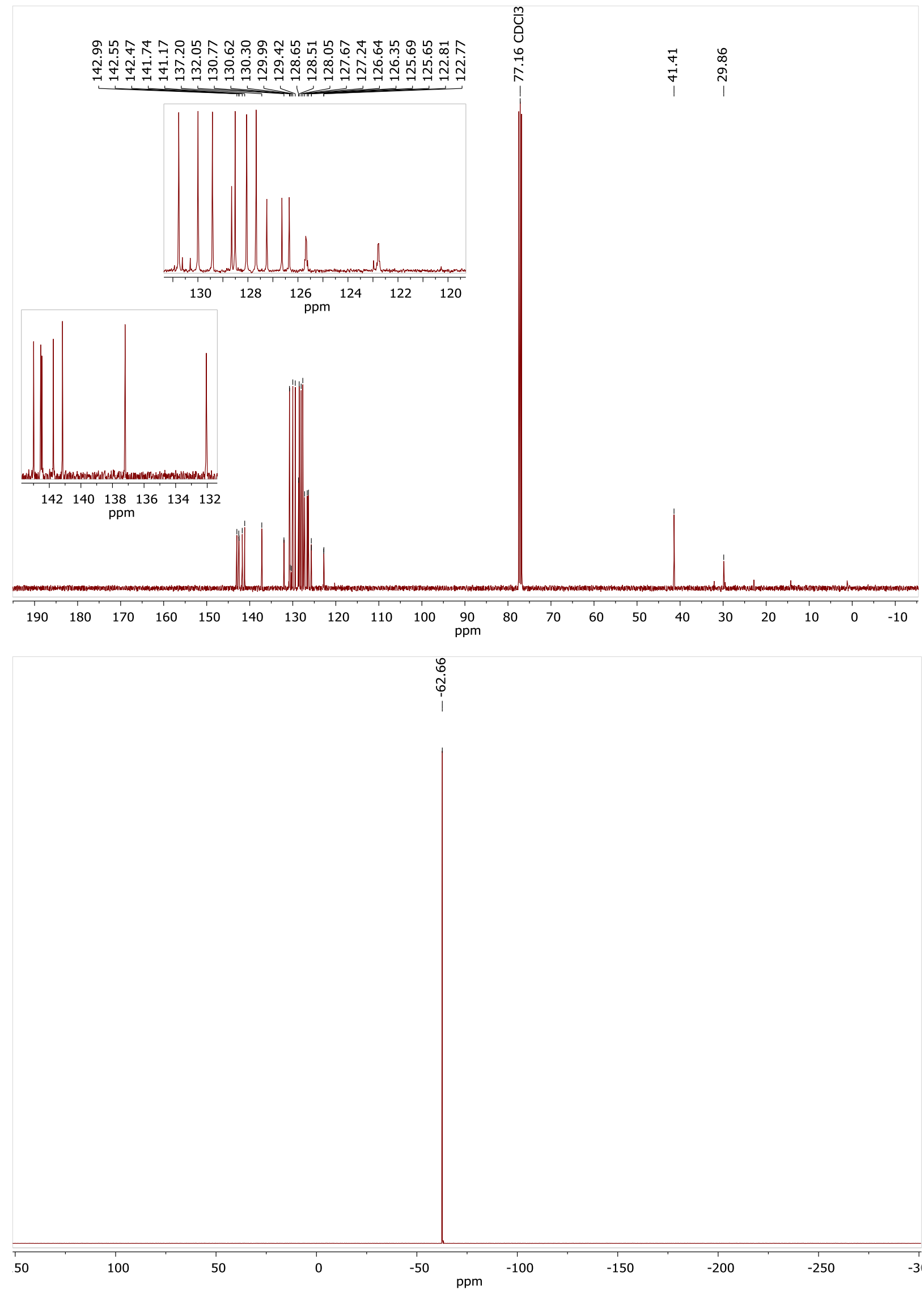


\section{${ }^{1} \mathrm{H}$ and ${ }^{13} \mathrm{C}$ NMR spectra of compound $4 \mathrm{I}$}

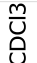

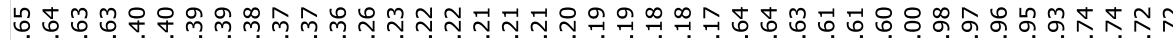

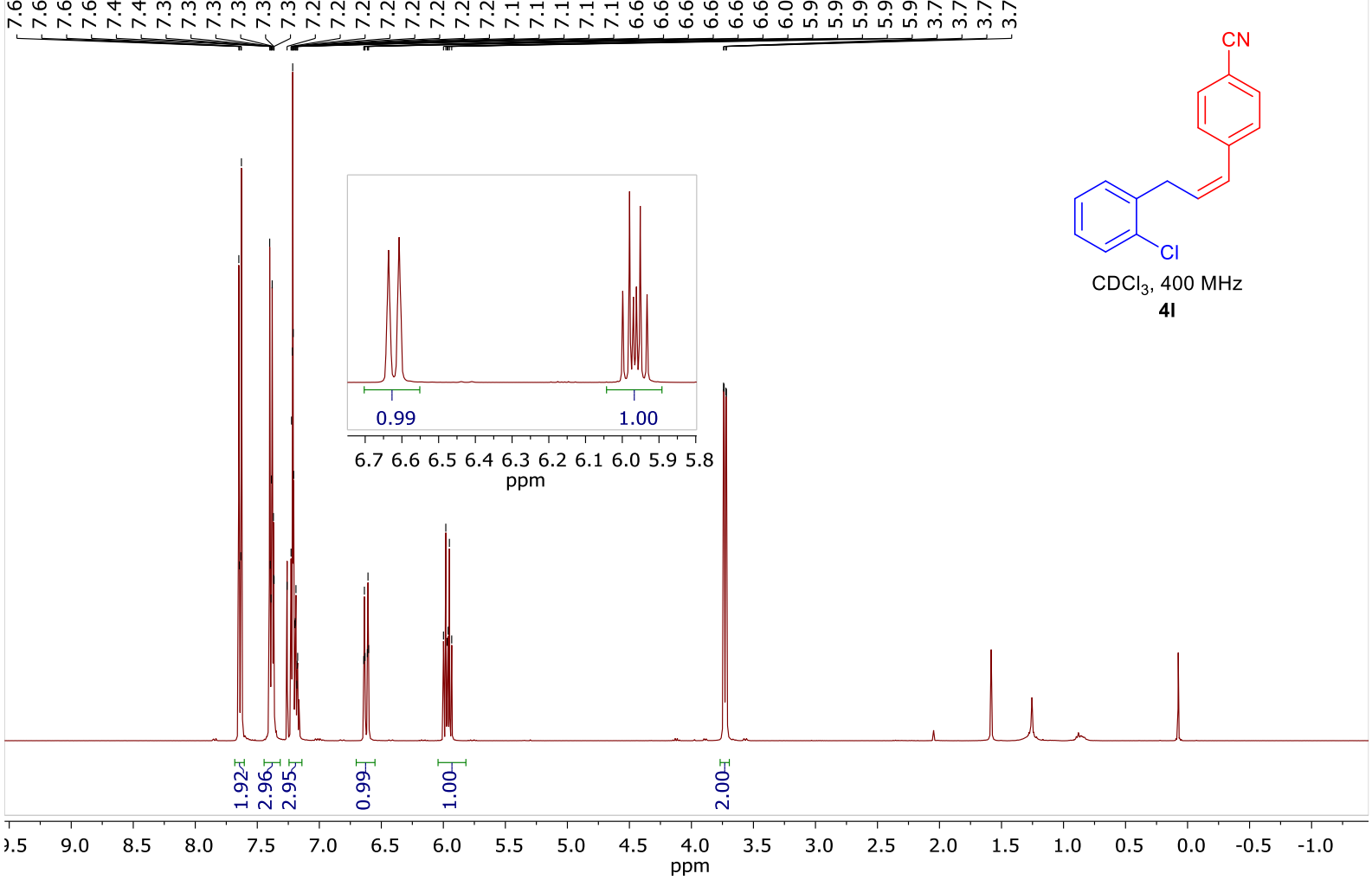

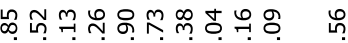

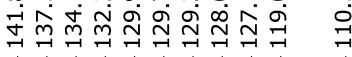

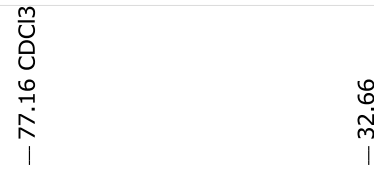

190180

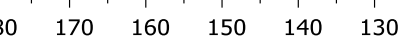

120

$110 \quad 100$ 
${ }^{1} \mathrm{H},{ }^{13} \mathrm{C}$ and ${ }^{19} \mathrm{~F}$ NMR spectra of compound $4 \mathrm{~m}$

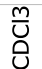

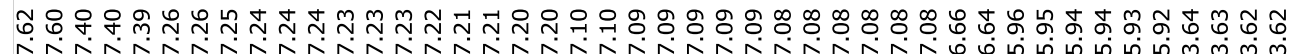
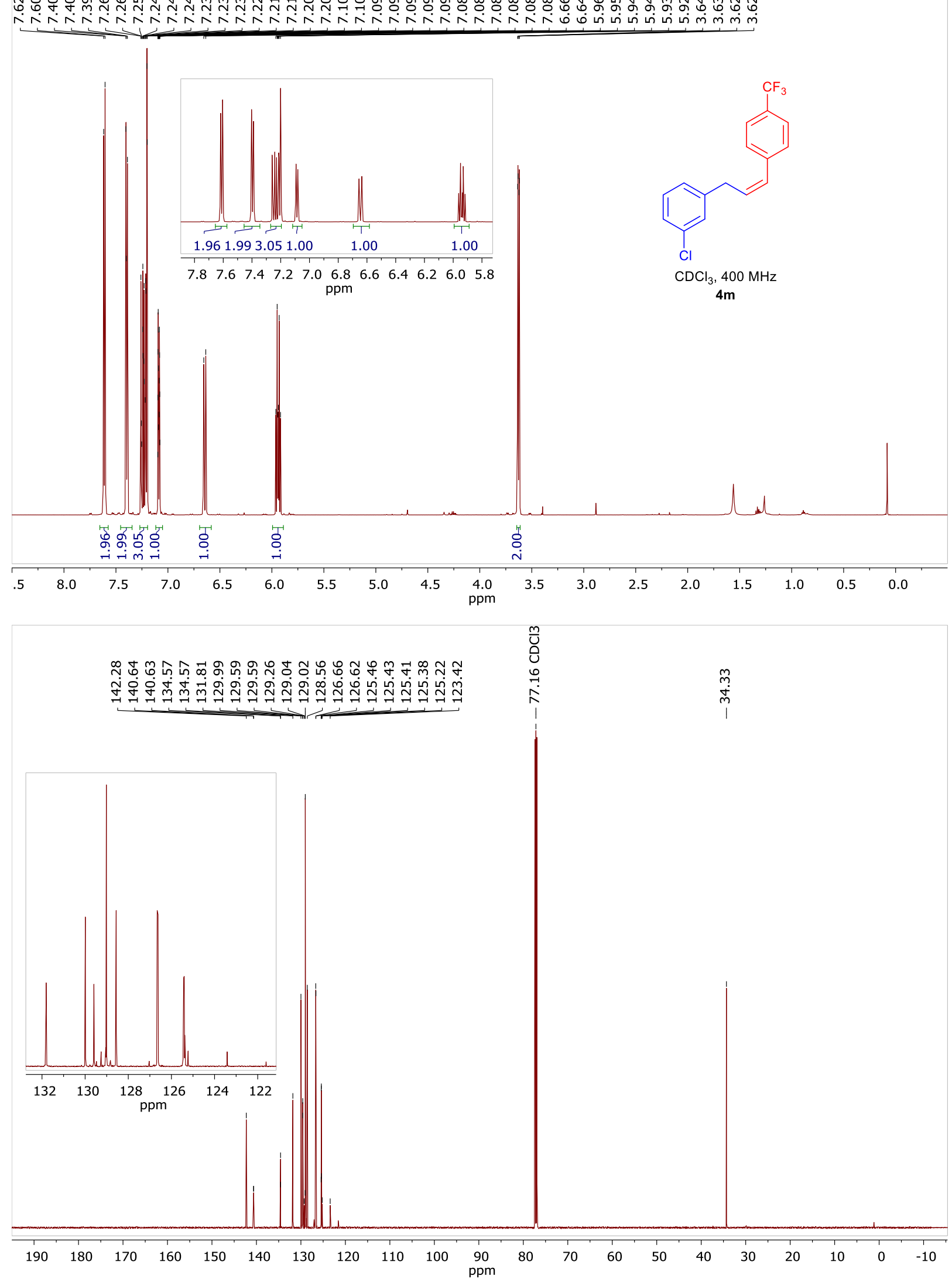


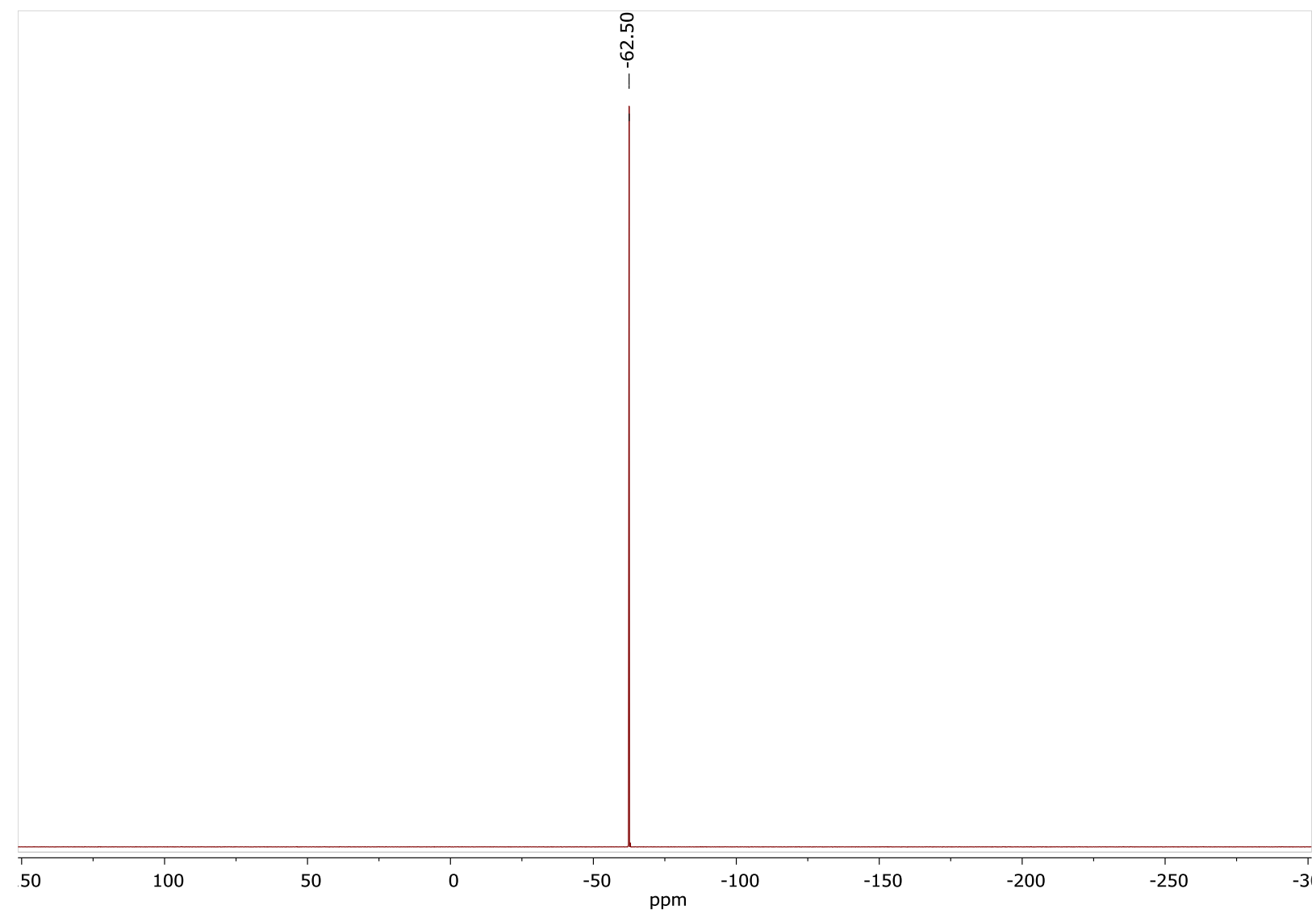

${ }^{1} \mathrm{H}$ and ${ }^{13} \mathrm{C}$ NMR spectra of compound $4 \mathbf{n}$

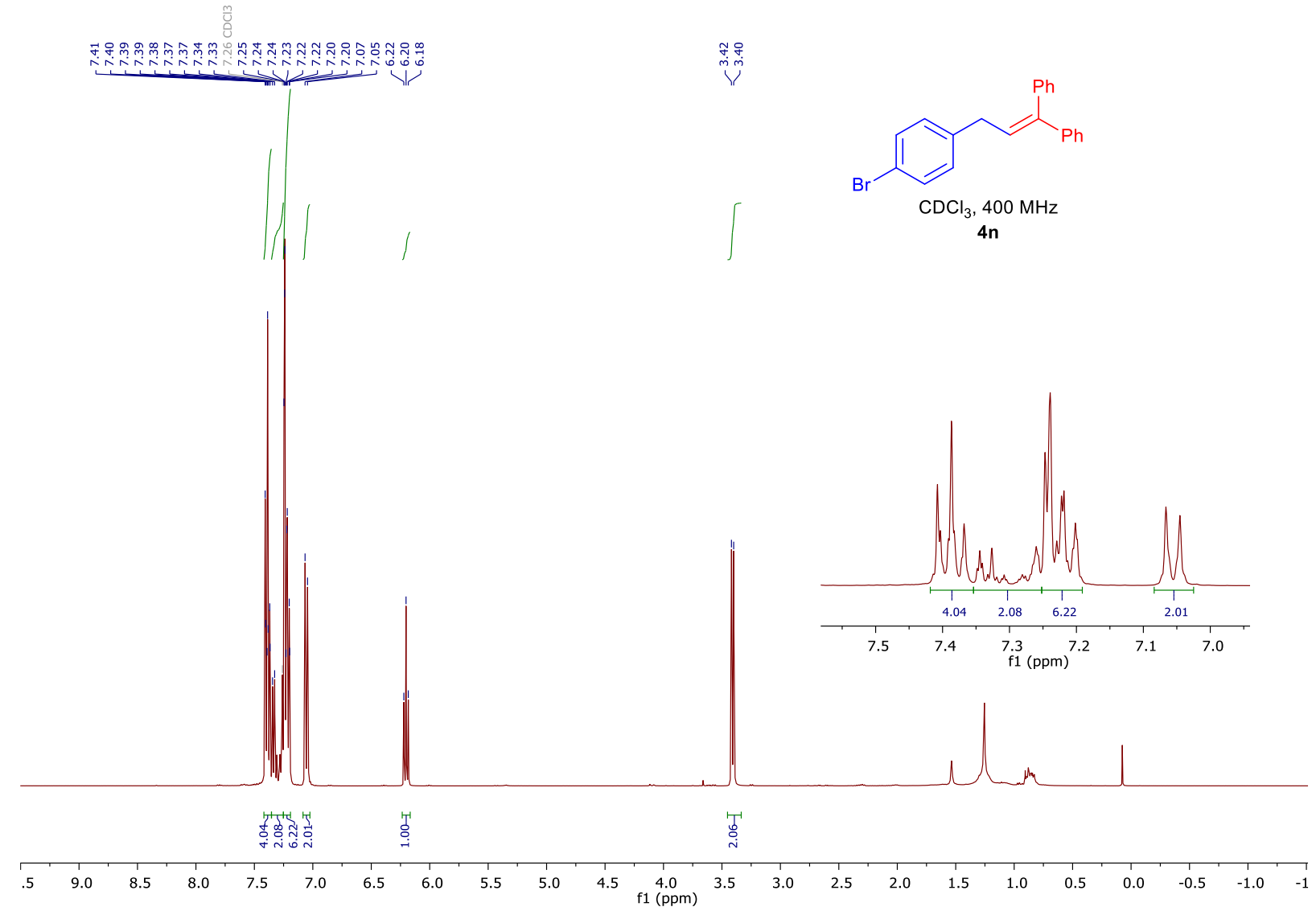




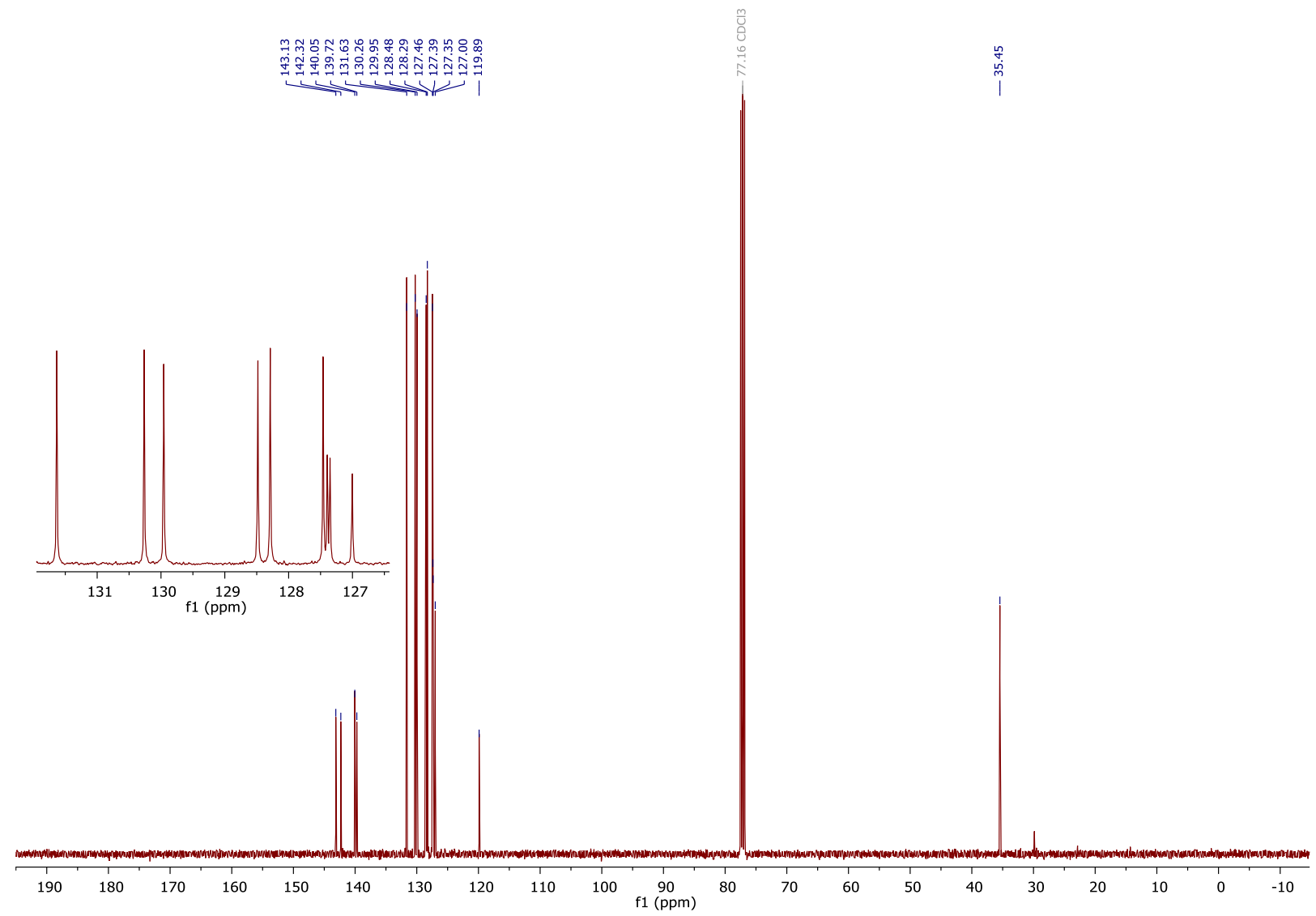

${ }^{1} \mathrm{H},{ }^{13} \mathrm{C}$ and ${ }^{19} \mathrm{~F}$ NMR spectra of compound 40

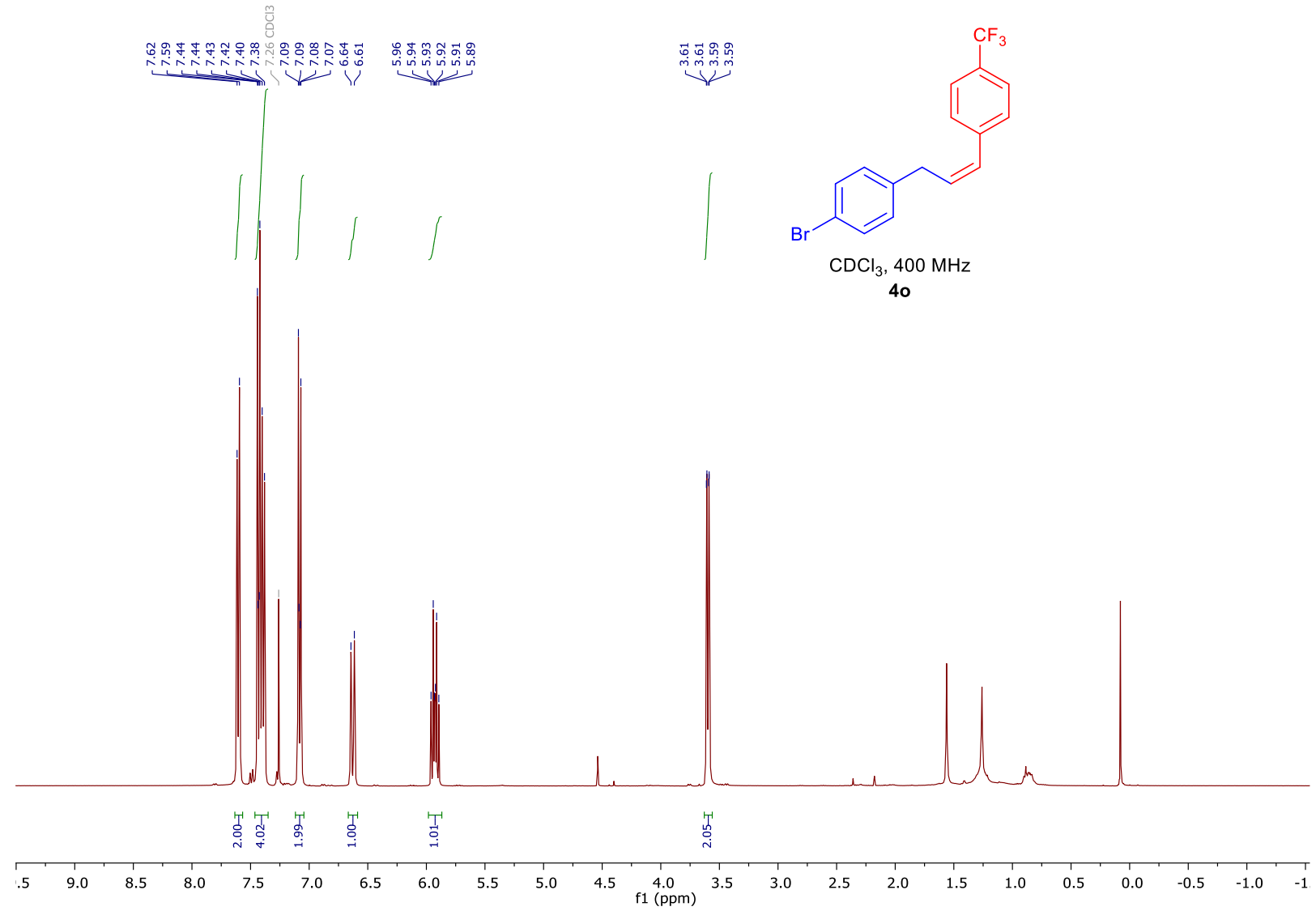




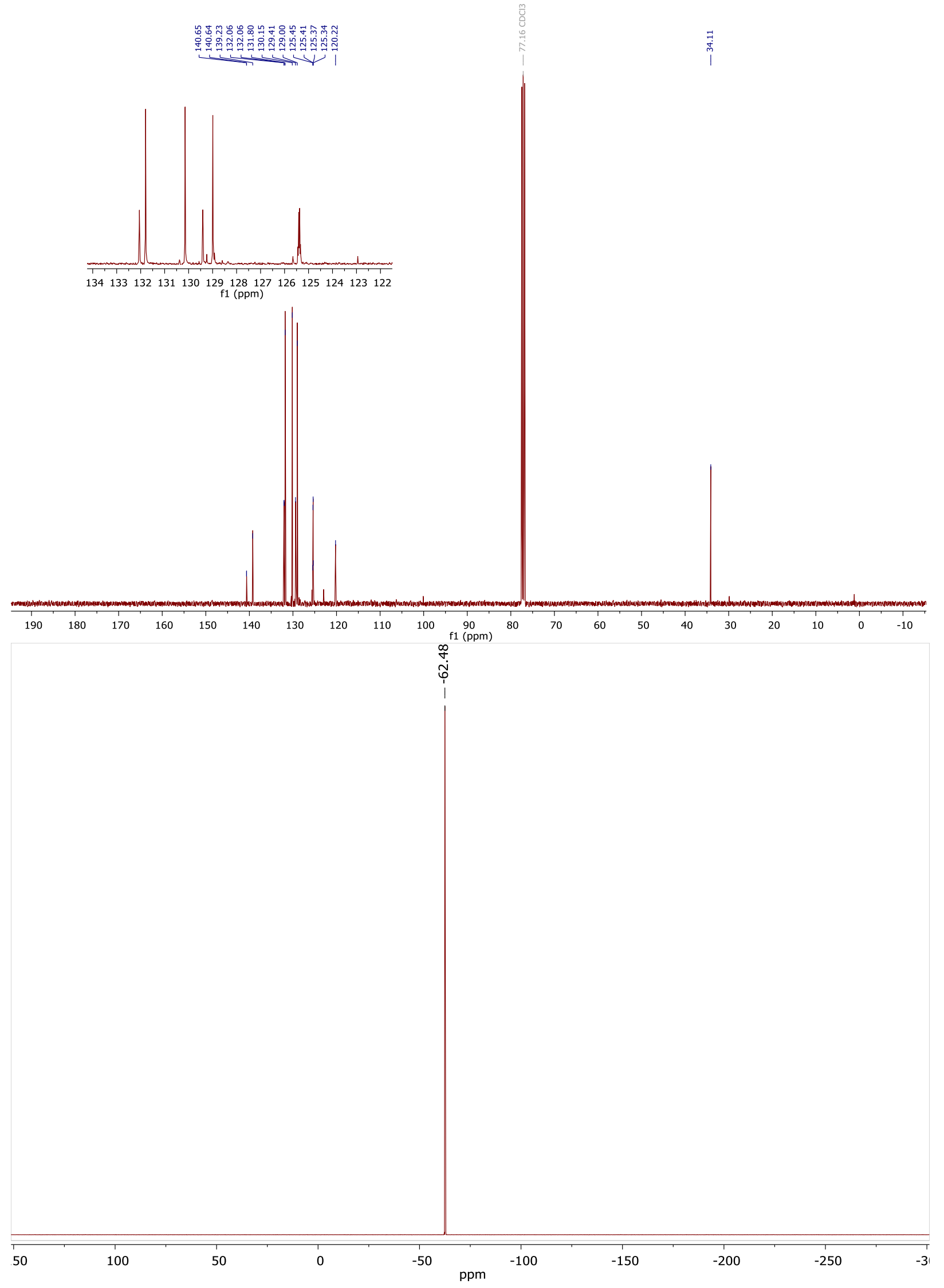


${ }^{1} \mathrm{H}$ and ${ }^{13} \mathrm{C}$ NMR spectra of compound $4 \mathrm{p}$

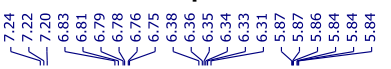
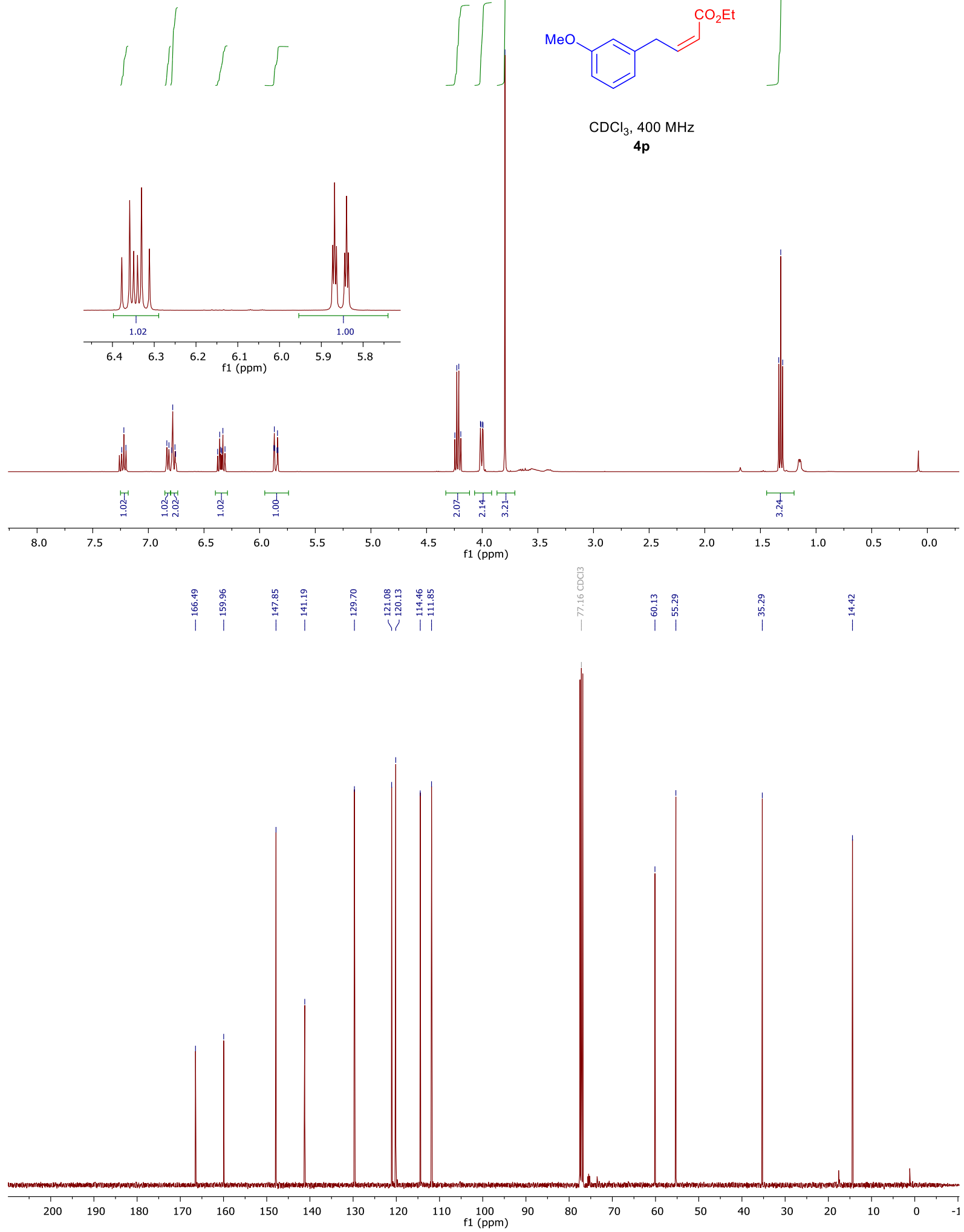

SI-50 
${ }^{1} \mathrm{H}$ and ${ }^{13} \mathrm{C}$ NMR spectra of compound $\mathbf{4 q}$

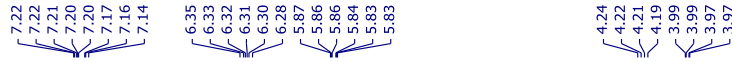

$\|$
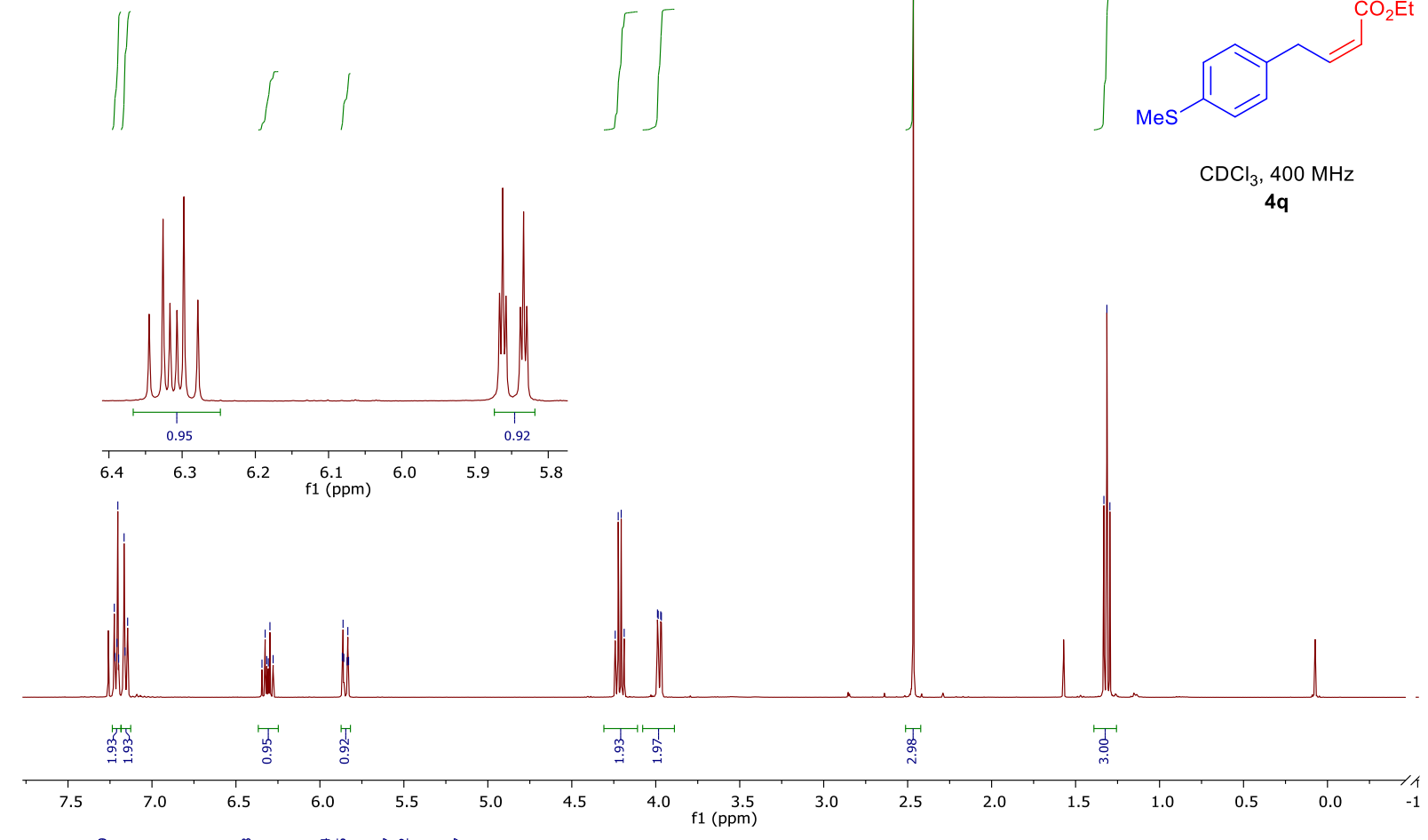

ำ

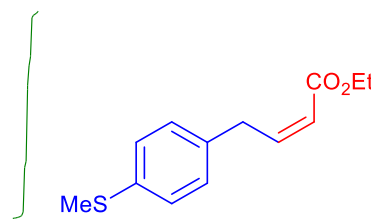

$\mathrm{CDCl}_{3}, 400 \mathrm{MHz}$

$4 q$

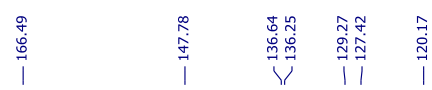

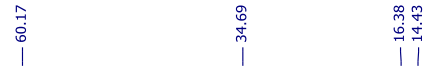

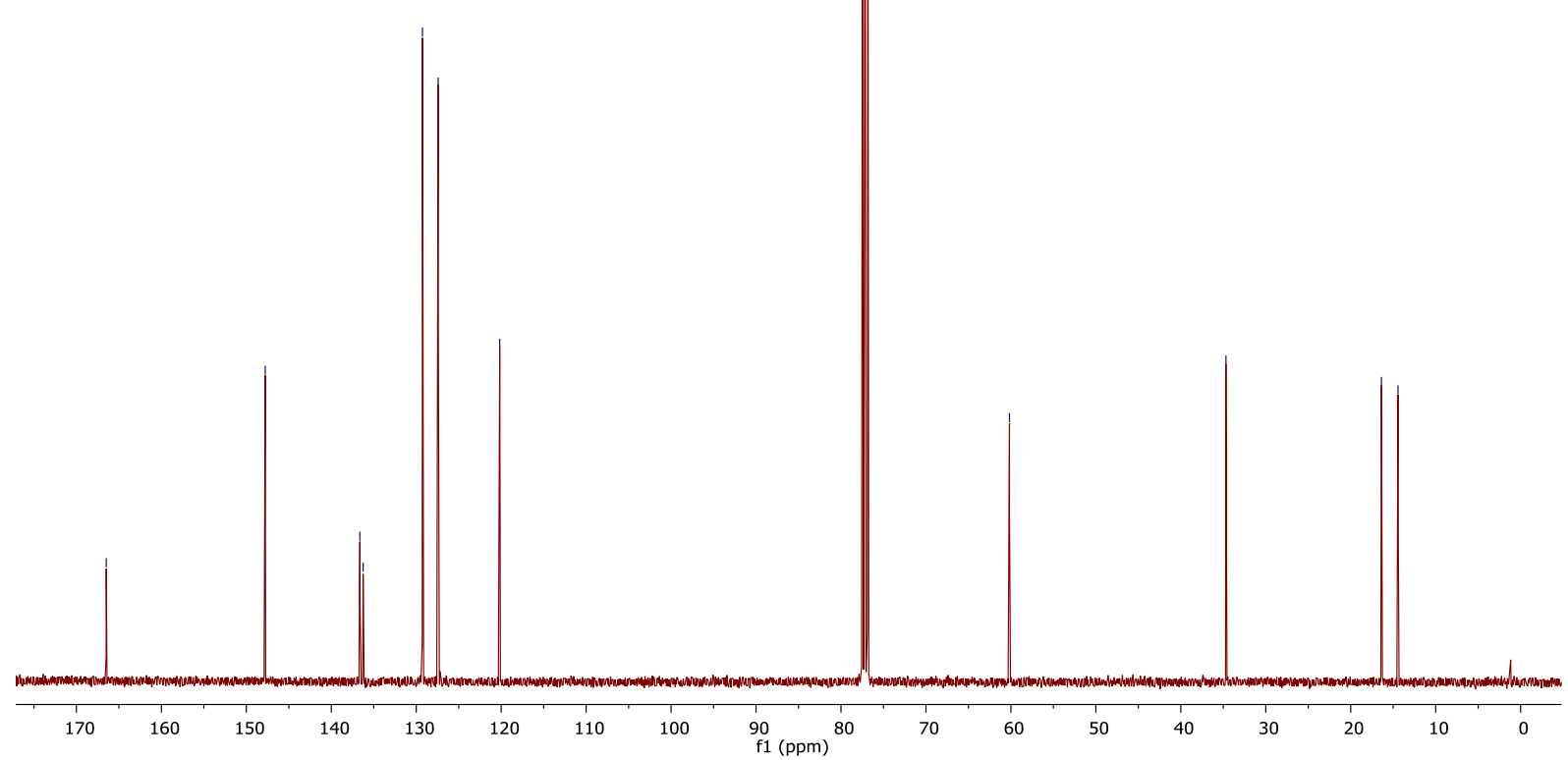


${ }^{1} \mathrm{H}$ and ${ }^{13} \mathrm{C}$ NMR spectra of compound $\mathbf{4 r}$
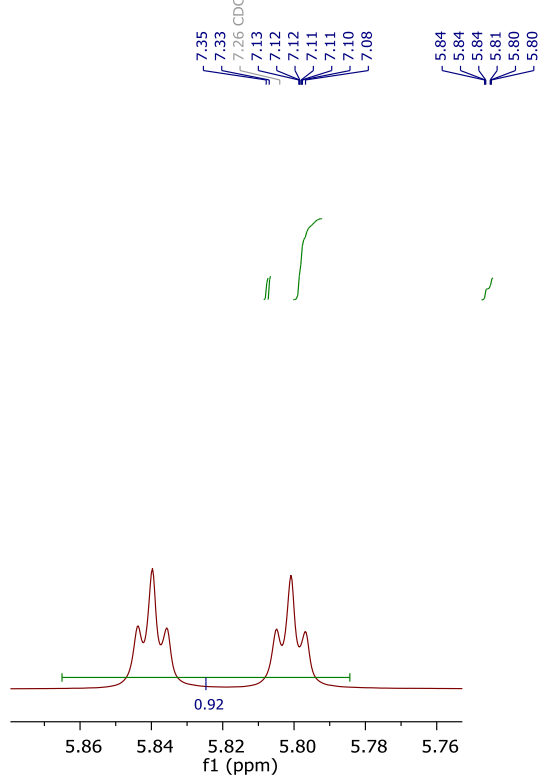

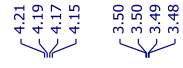

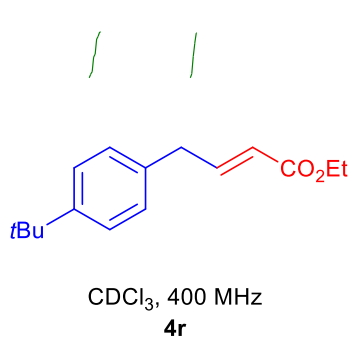

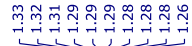

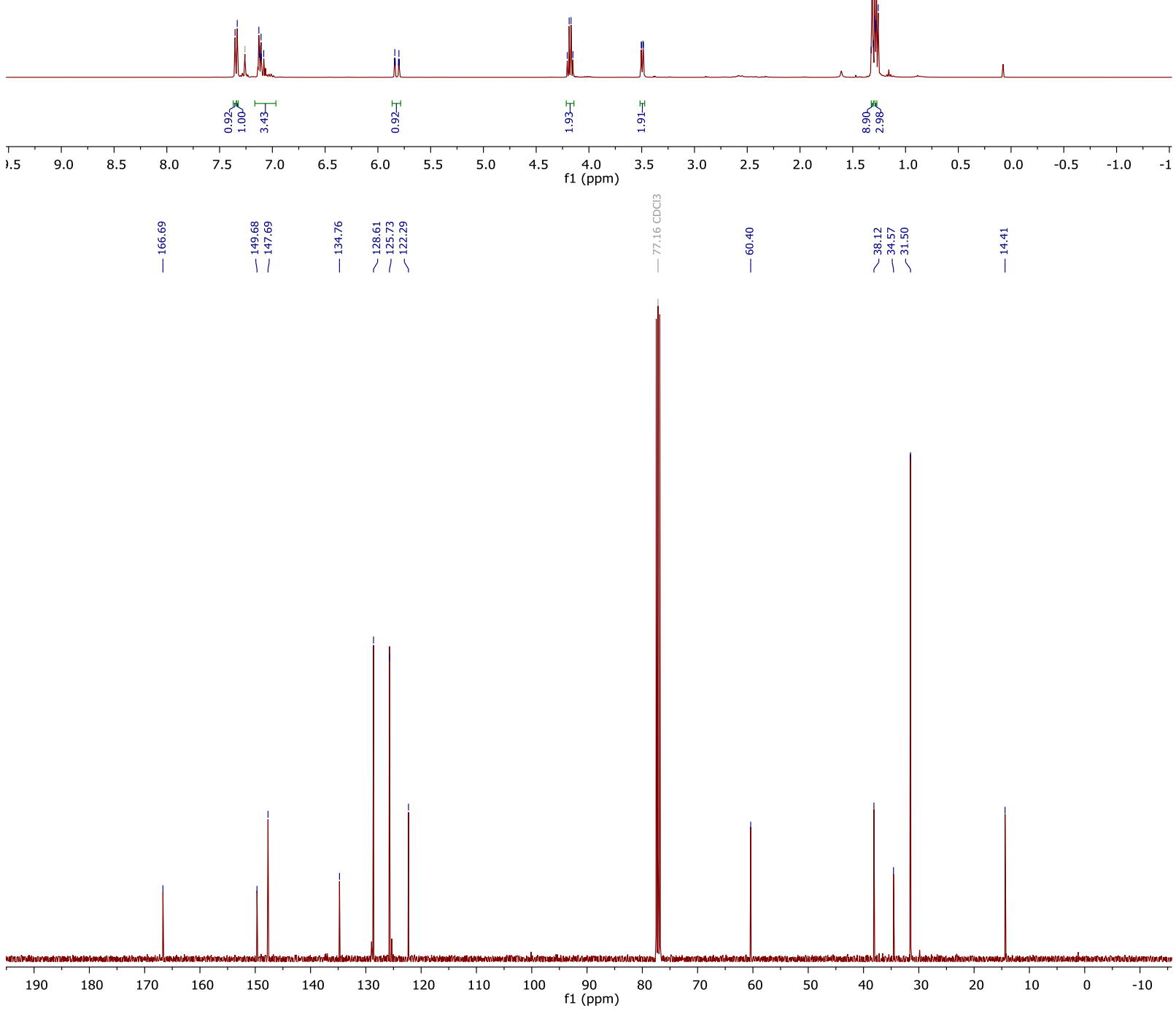

SI-52 
${ }^{1} \mathrm{H},{ }^{13} \mathrm{C}$ and NOESY NMR spectra of compound $4 \mathbf{s}$
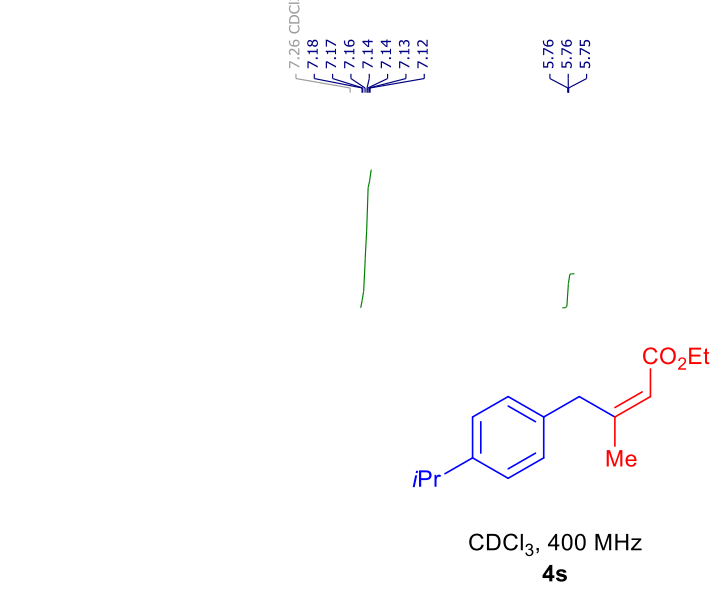
$\mathrm{CDCl}_{3}, \underset{4 \mathbf{s}}{400 \mathrm{MHz}}$

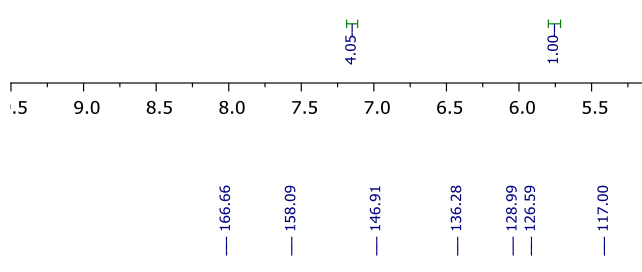

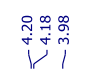
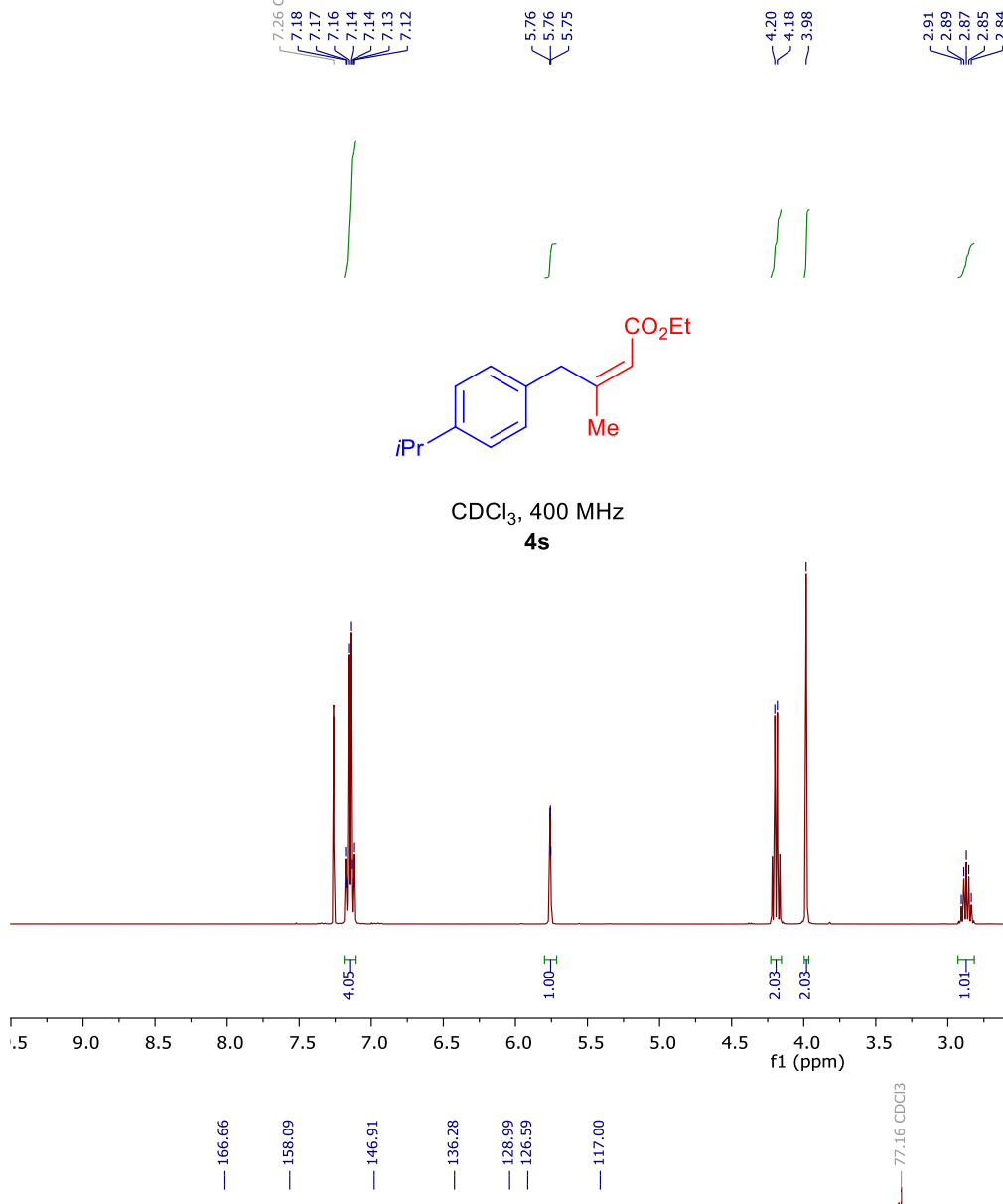
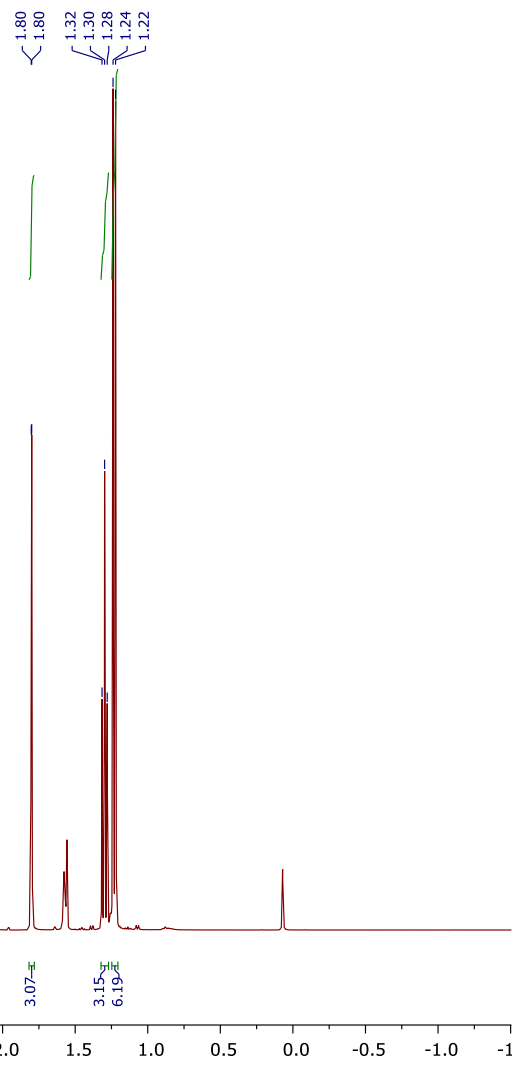

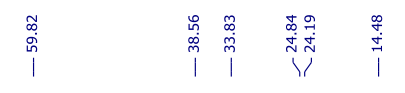

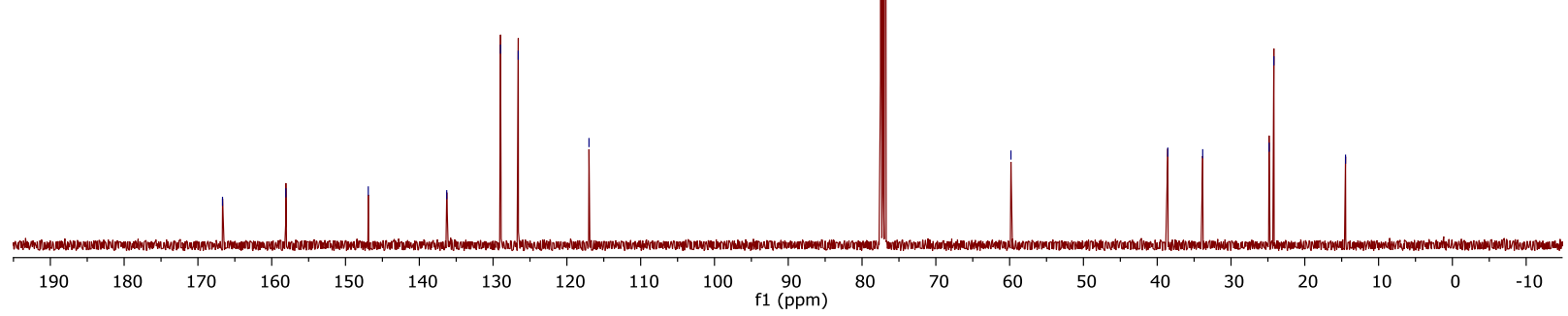

SI-53 


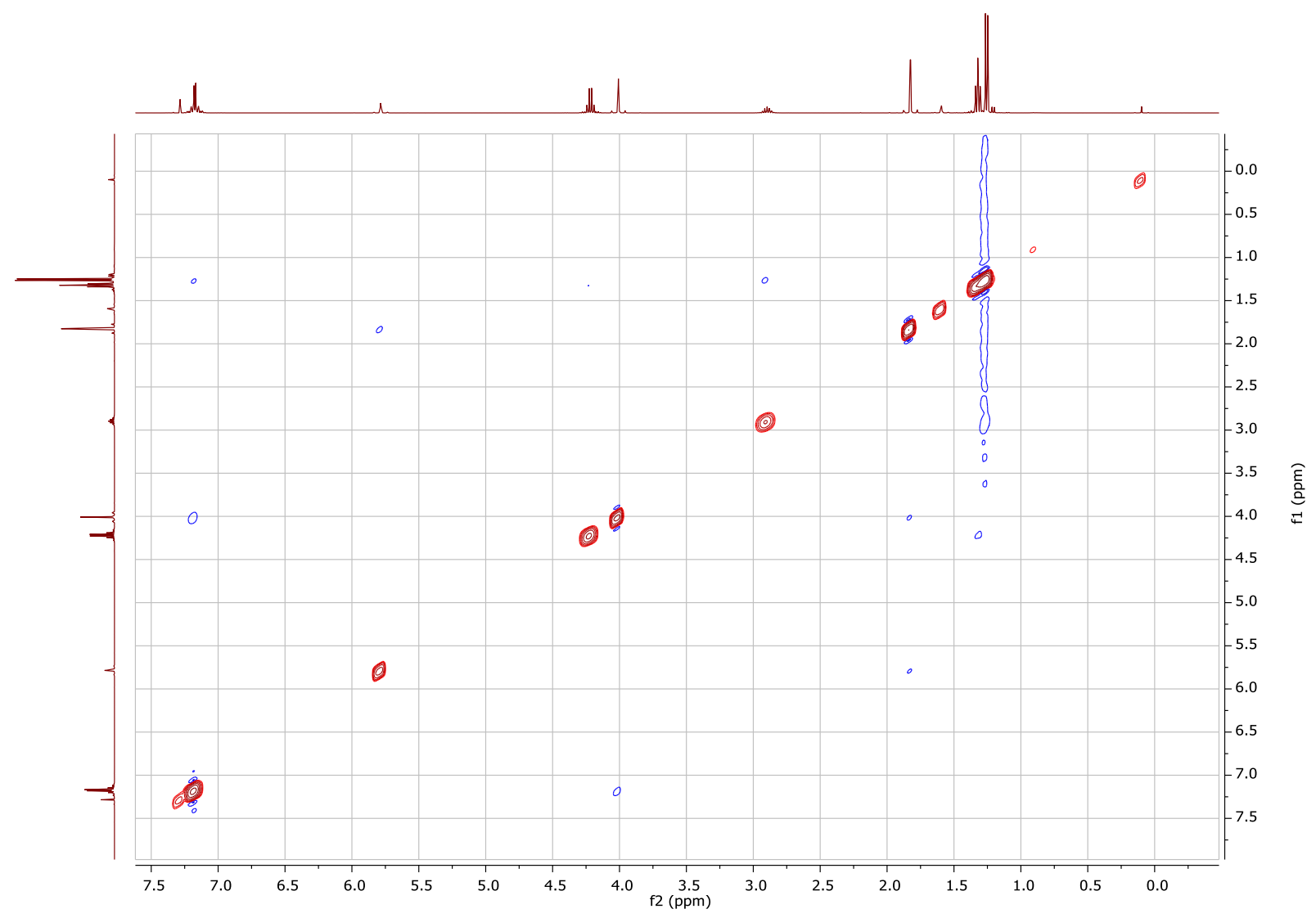

${ }^{1} \mathrm{H},{ }^{13} \mathrm{C}$ and ${ }^{19} \mathrm{~F}$ NMR spectra of compound $4 \mathbf{t}$

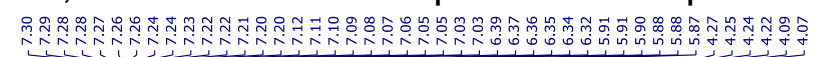

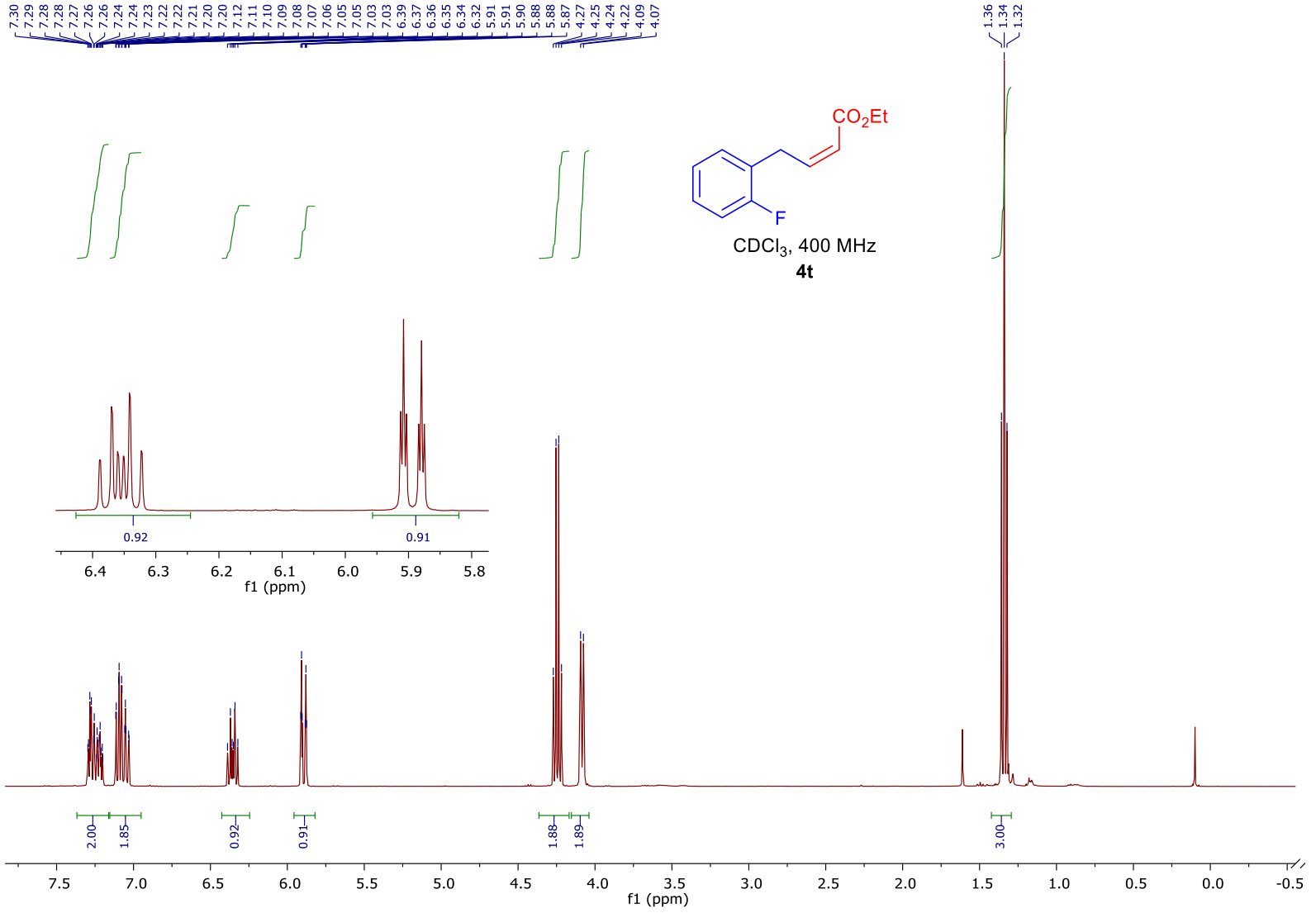




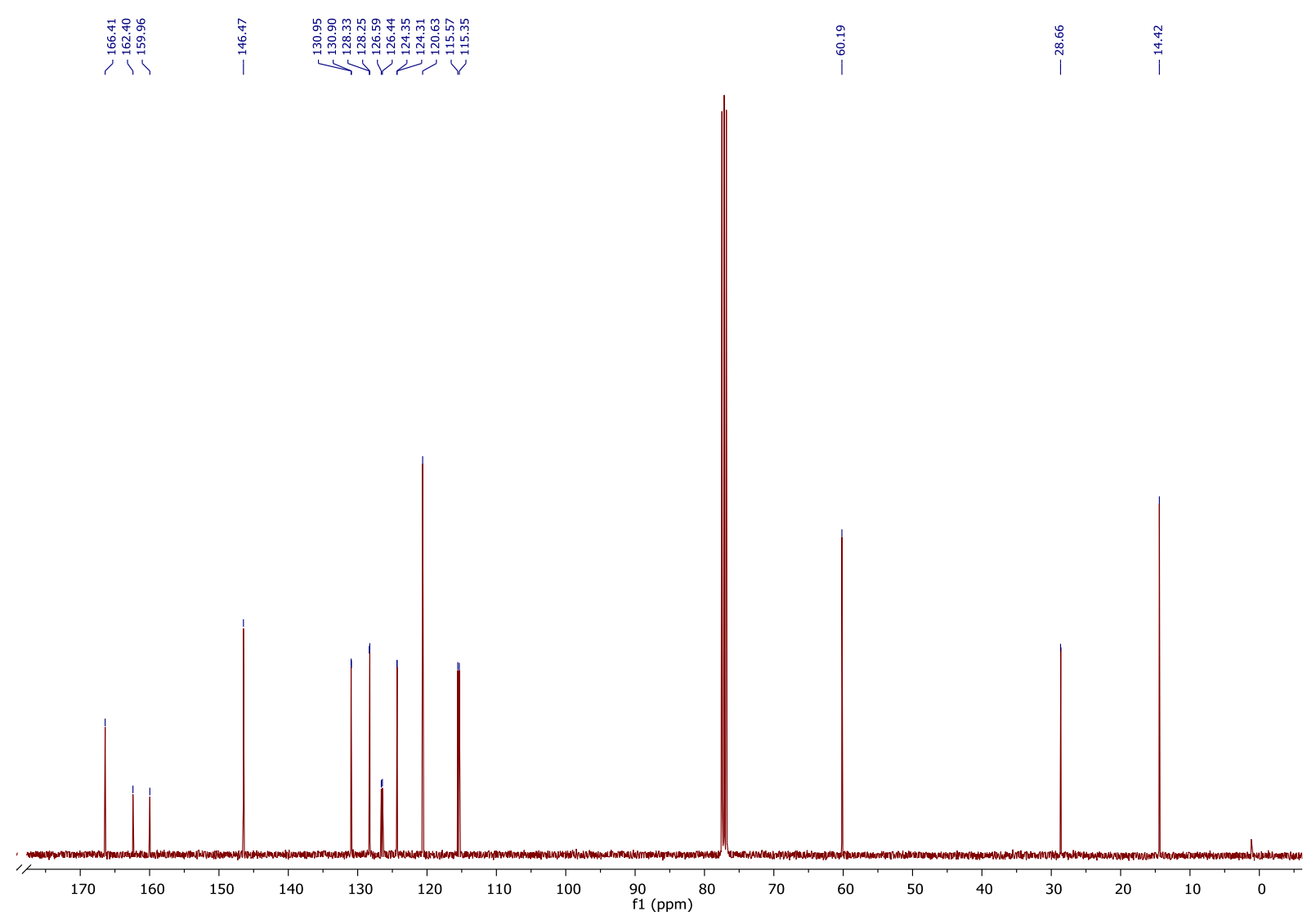

\begin{tabular}{|c|c|c|}
\hline 50 & 100 & 50 \\
\hline
\end{tabular}




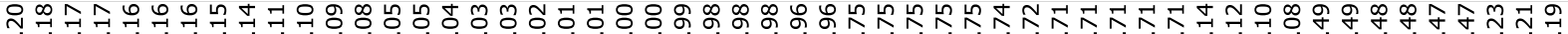

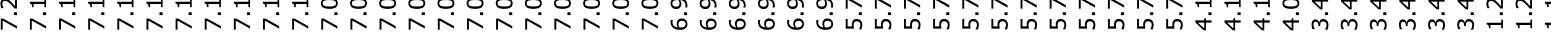

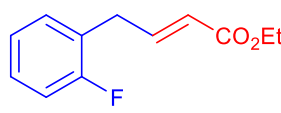

$\mathrm{CDCl}_{3}, 400 \mathrm{MHz}$

$4 \mathrm{u}$
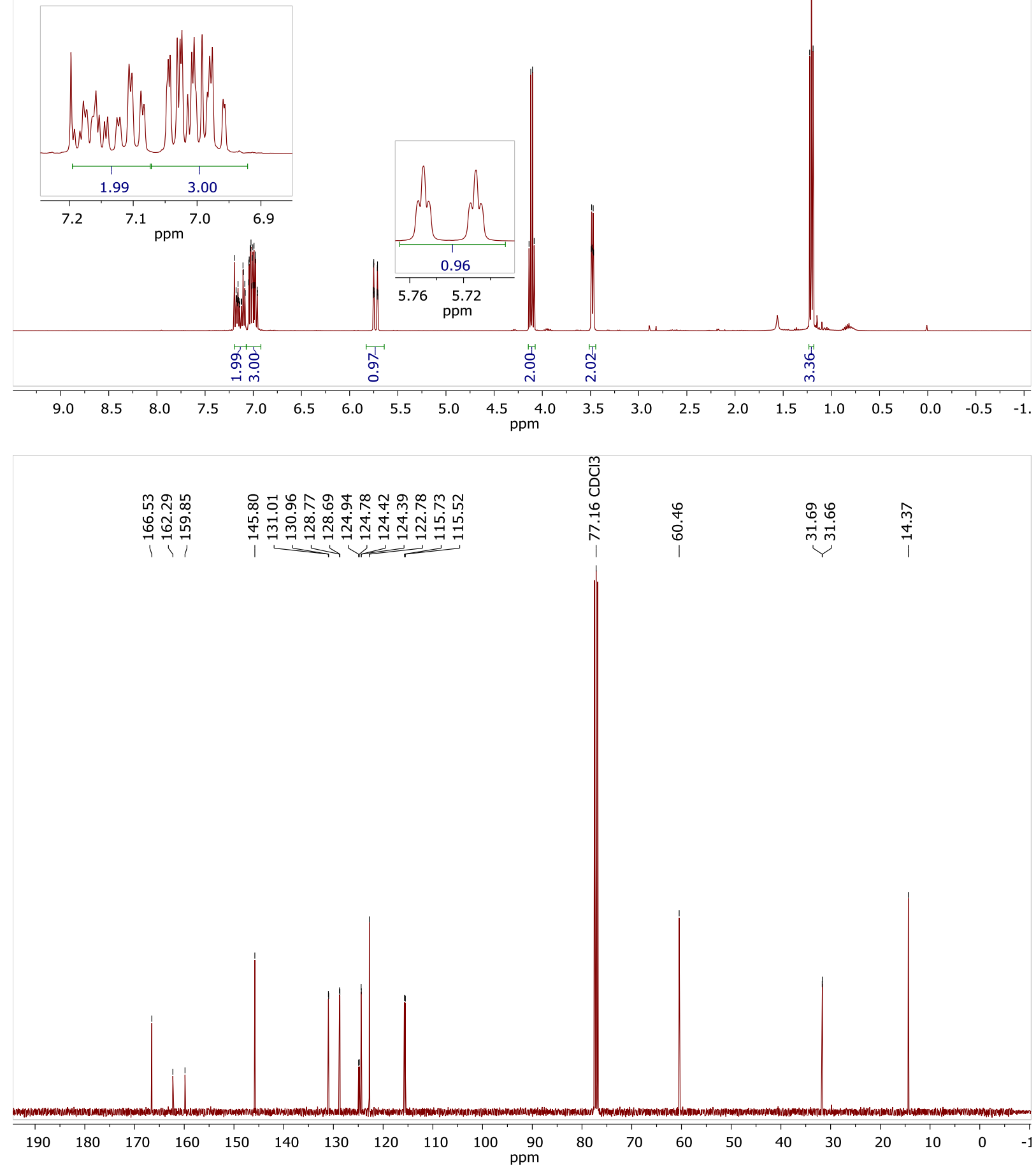


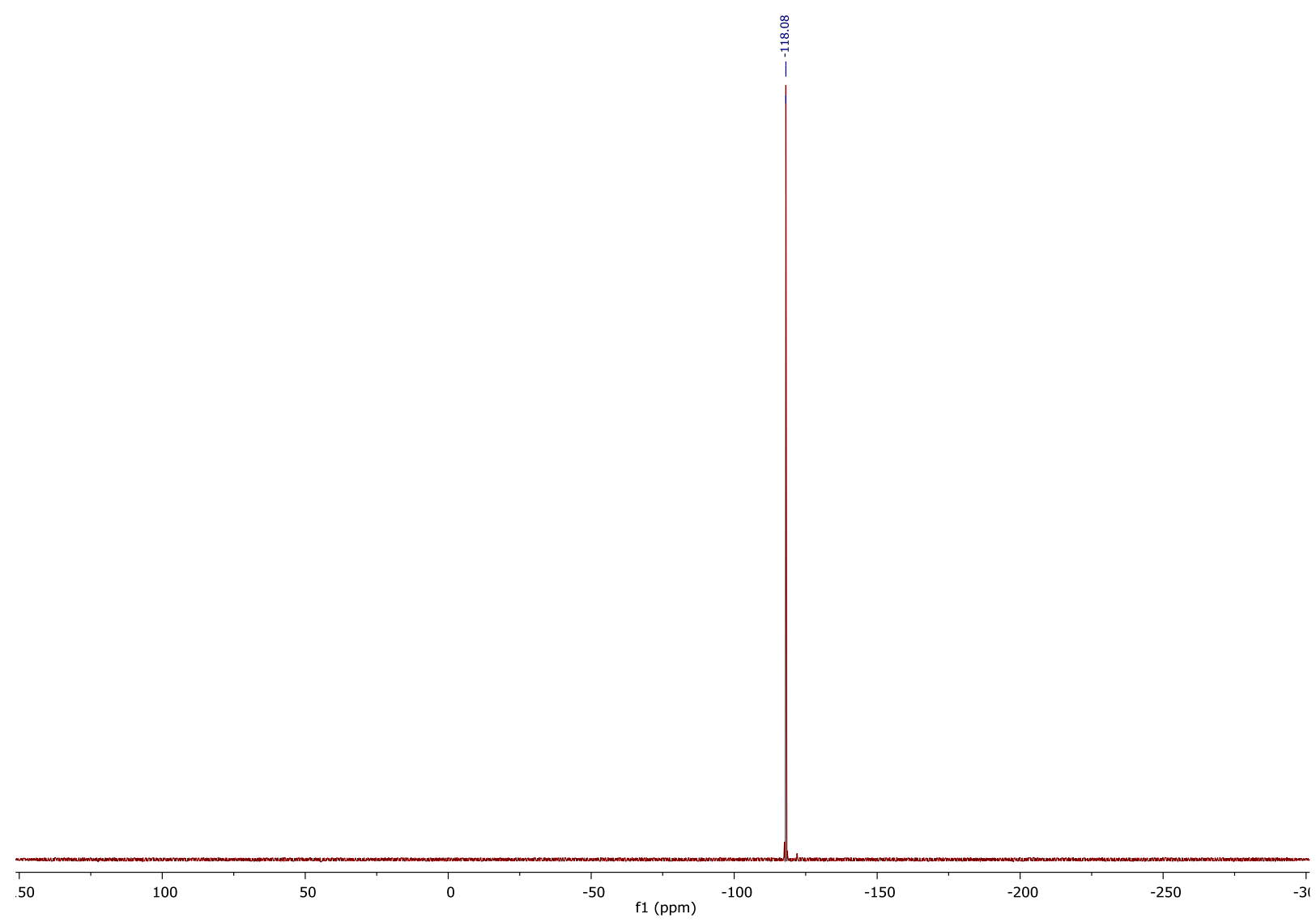

${ }^{1} \mathrm{H},{ }^{13} \mathrm{C}$ and ${ }^{19} \mathrm{~F}$ NMR spectra of compound $\mathbf{4} \mathbf{v}$

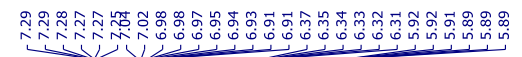

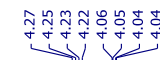
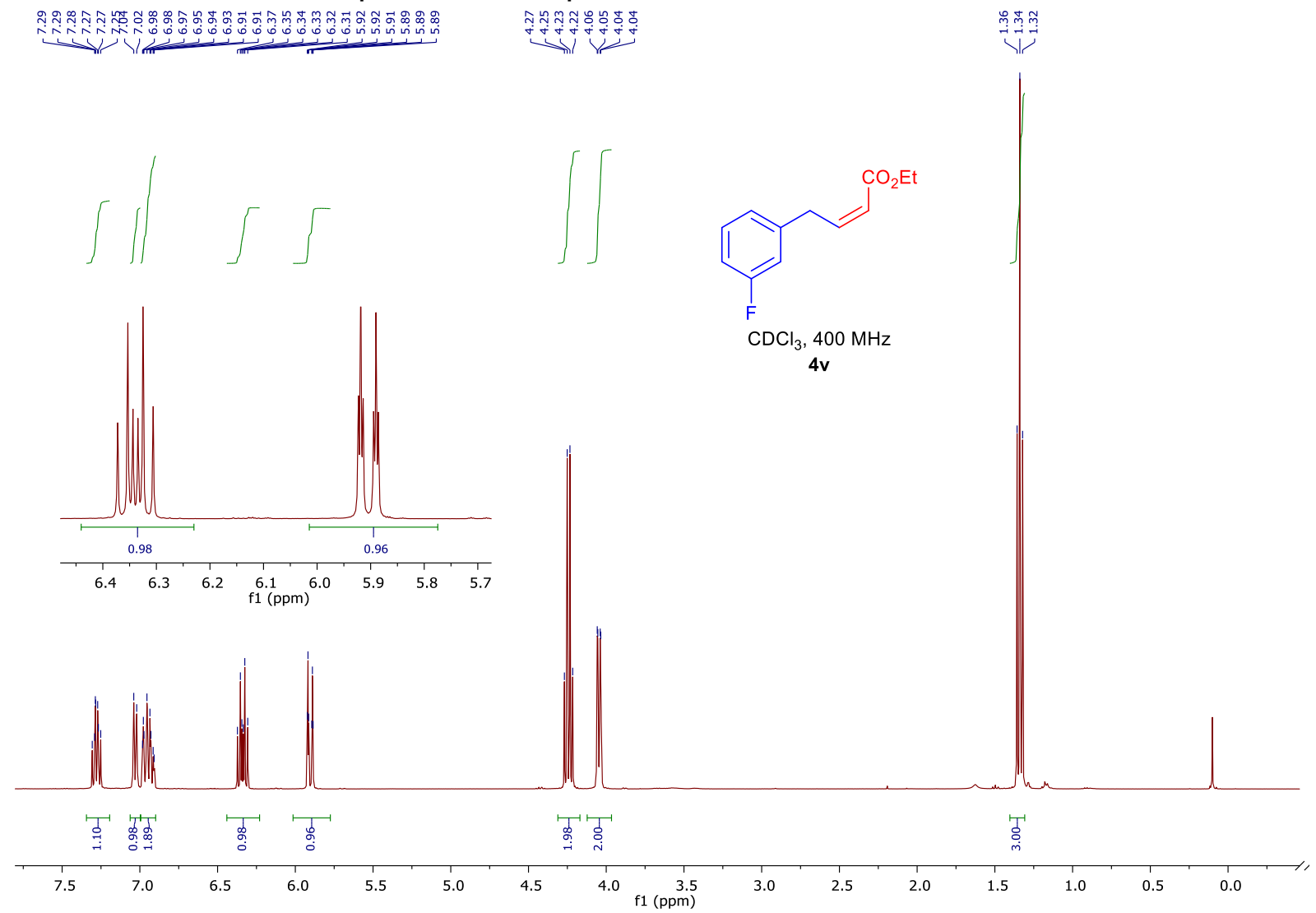


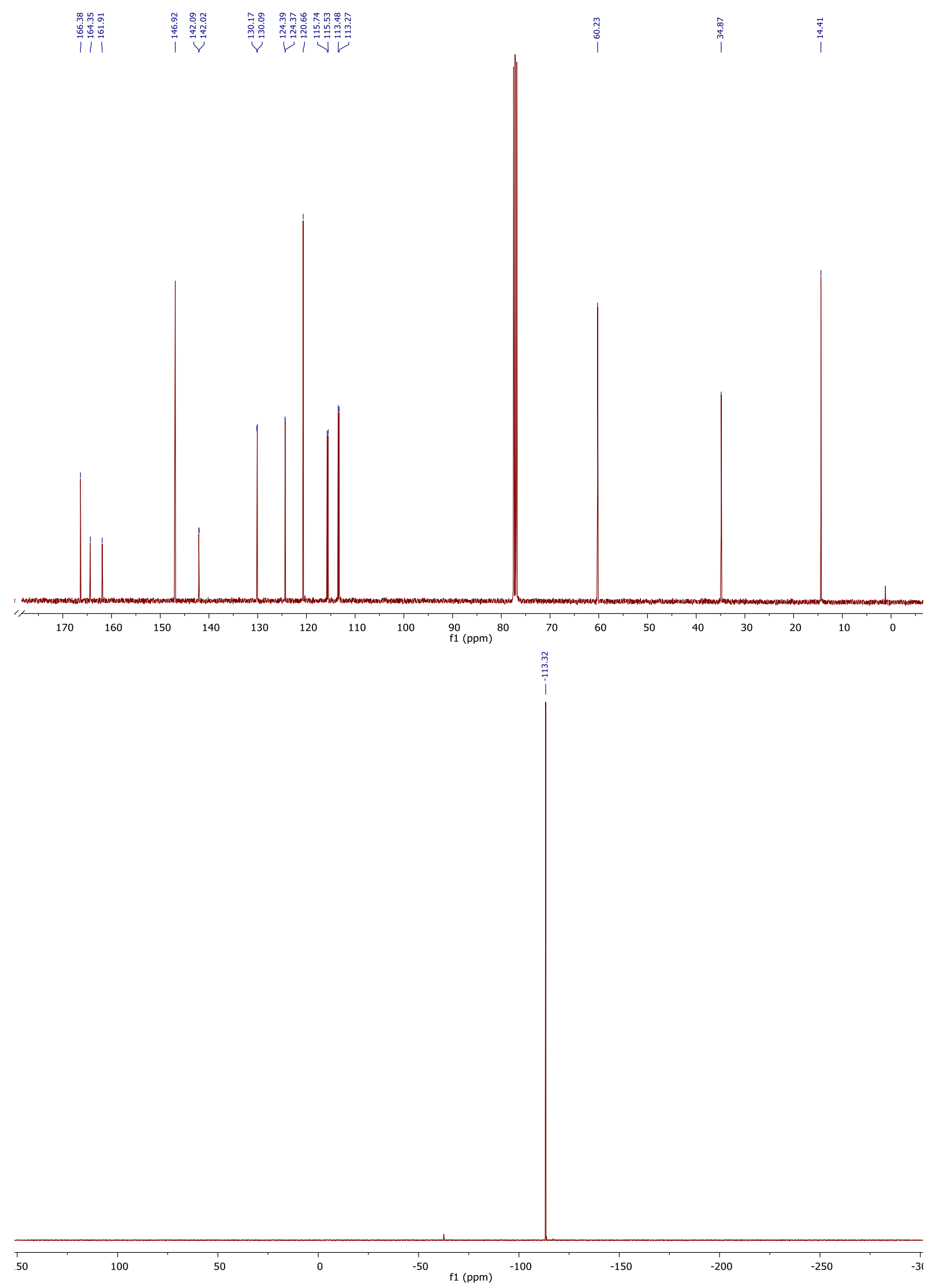


${ }^{1} \mathrm{H},{ }^{13} \mathrm{C},{ }^{19} \mathrm{~F}$ and NOESY NMR spectra of compound $4 \mathbf{w}$ 范

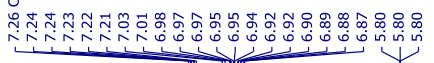
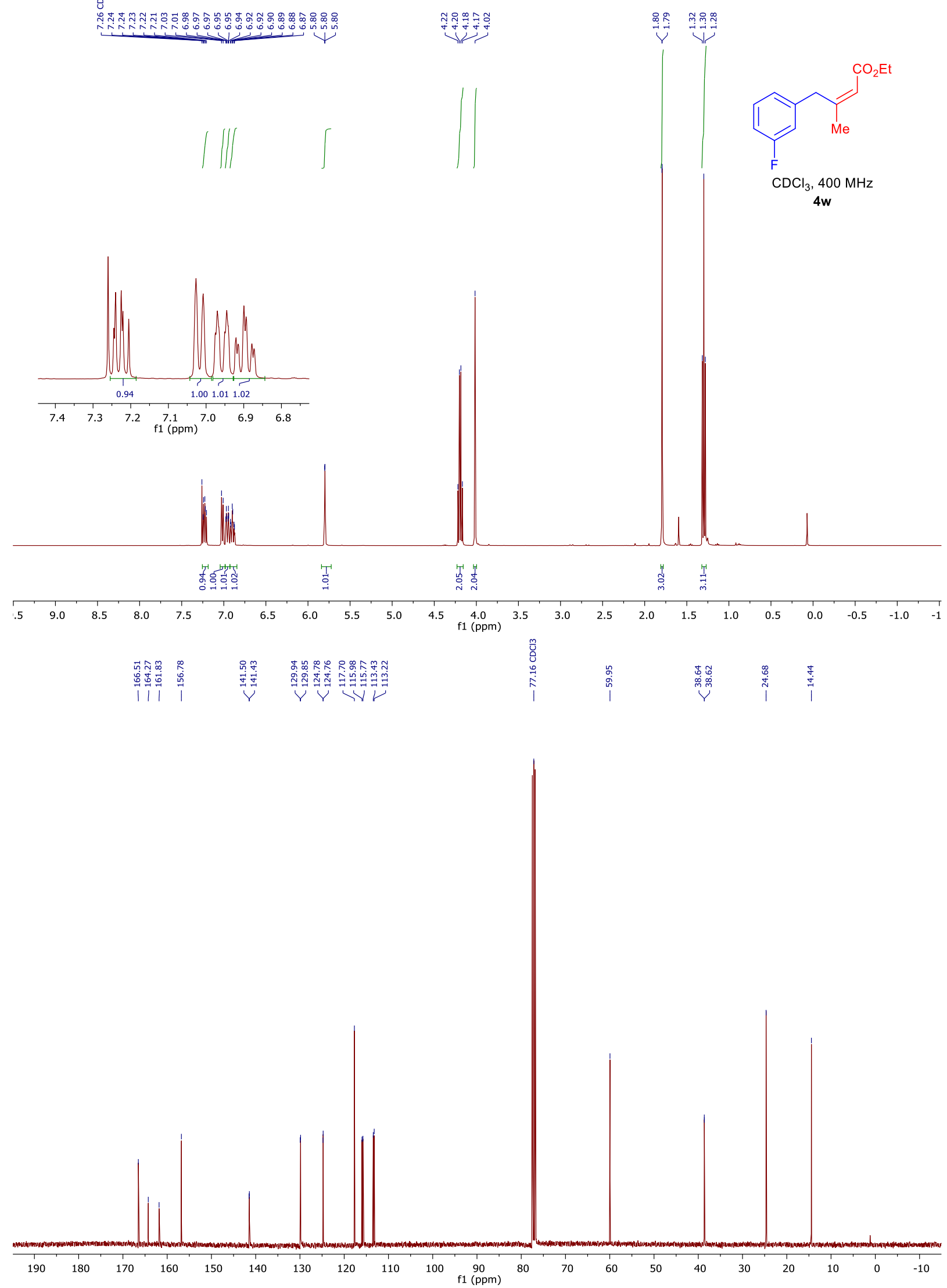


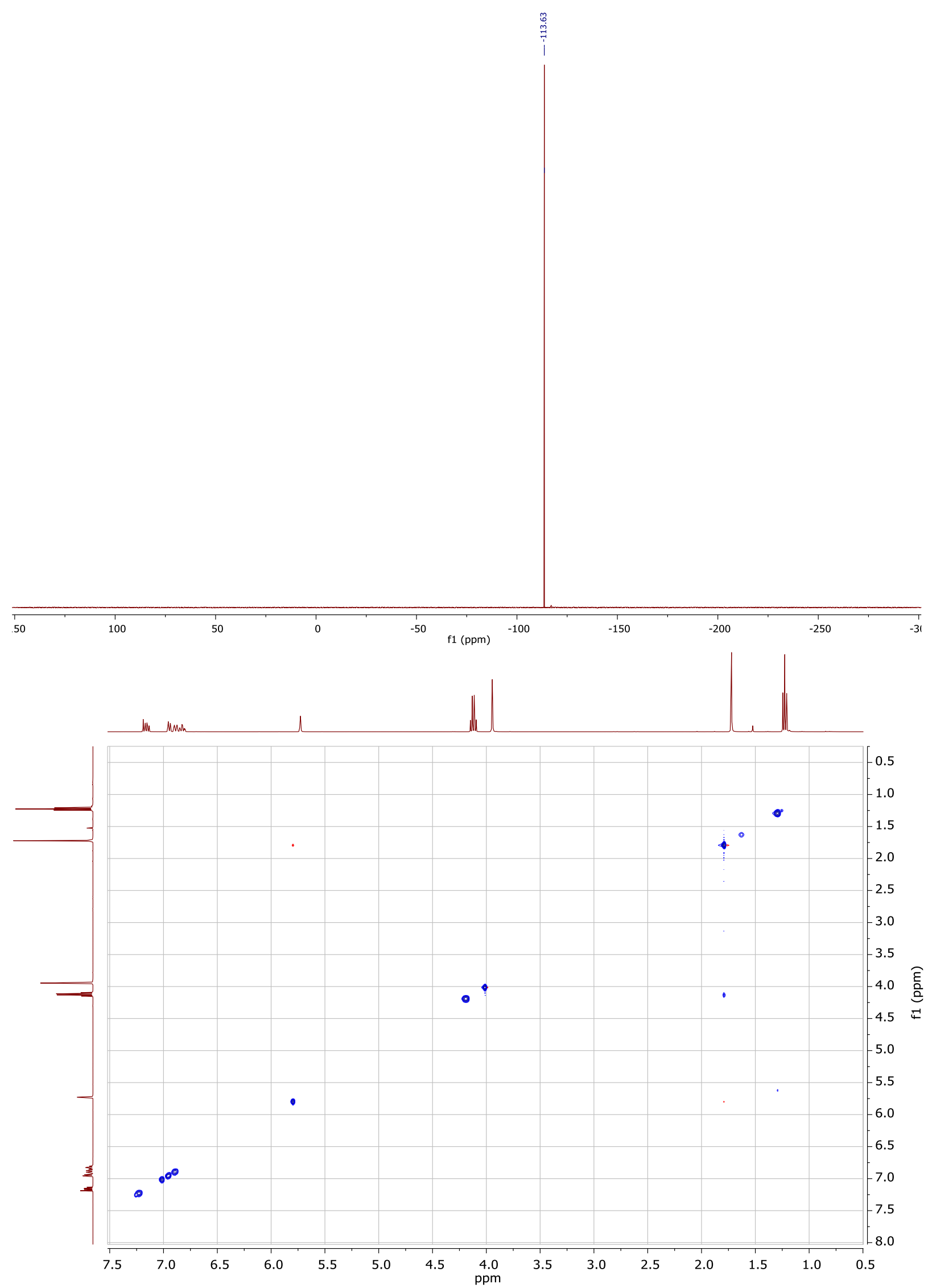


${ }^{1} \mathrm{H},{ }^{13} \mathrm{C}$ and ${ }^{19} \mathrm{~F}$ NMR spectra of compound $\mathbf{4 x}$

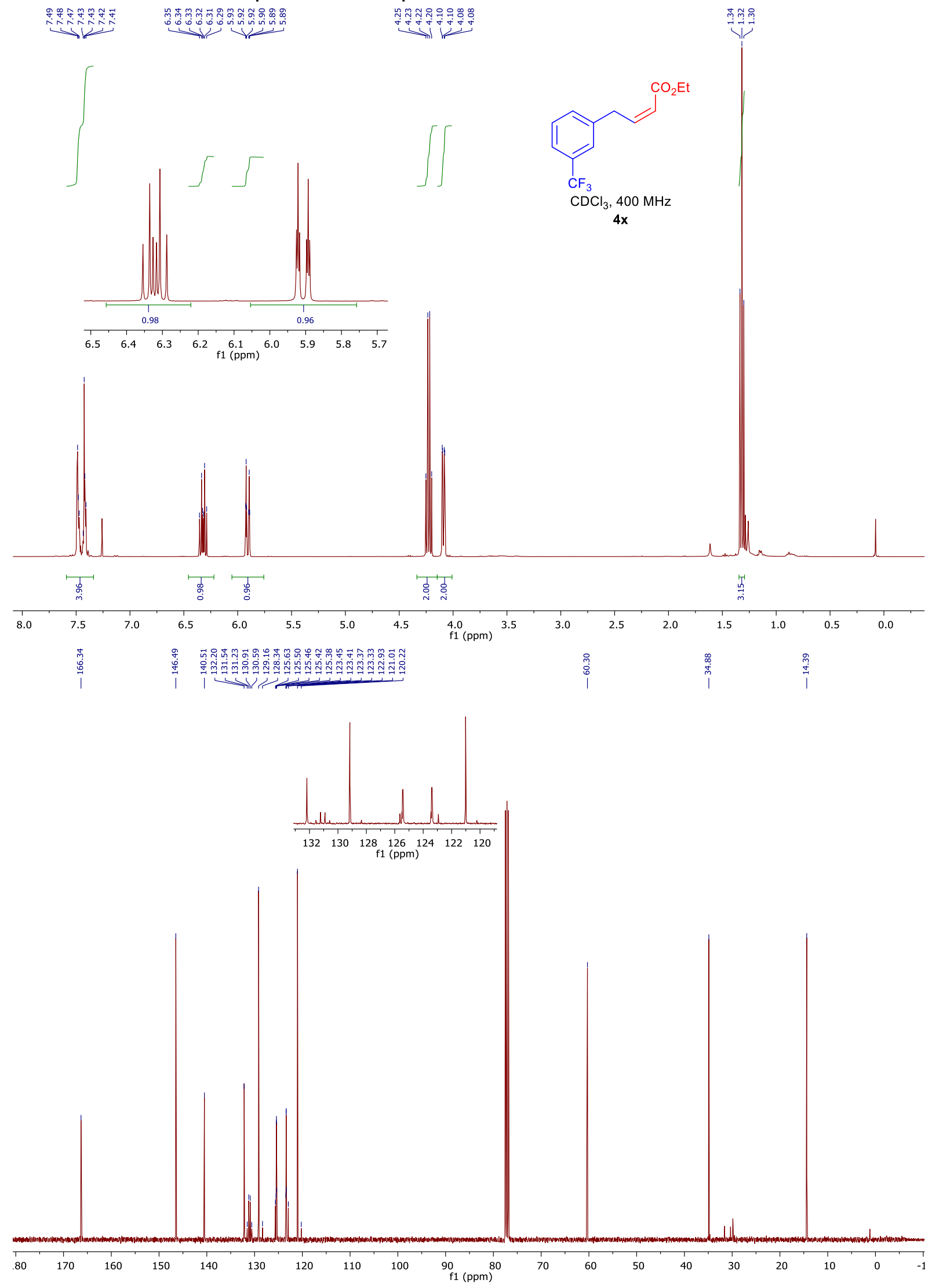




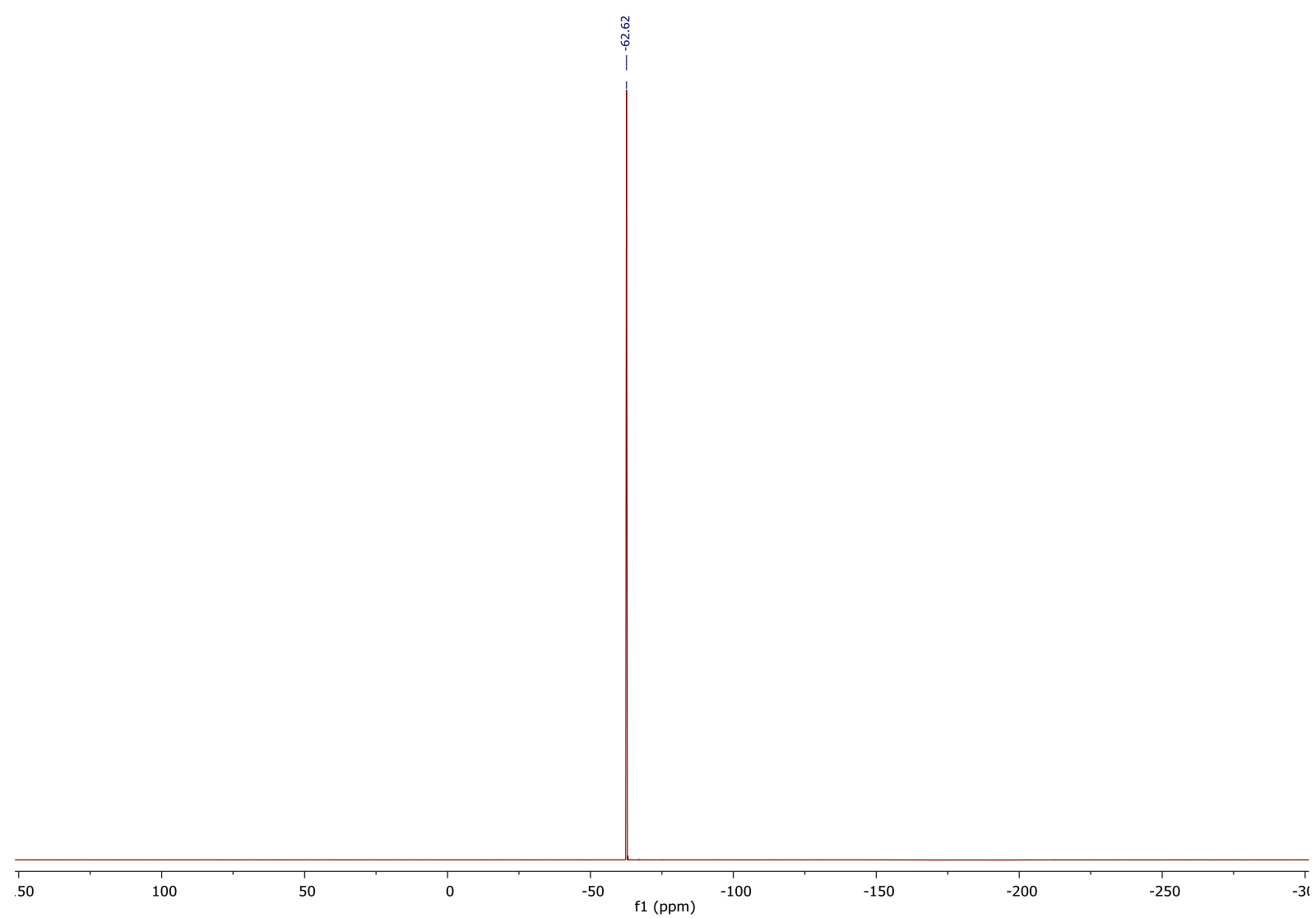

\section{${ }^{1} \mathrm{H}$ and ${ }^{13} \mathrm{C}$ spectra of compound $4 \mathbf{y}$}

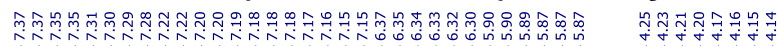

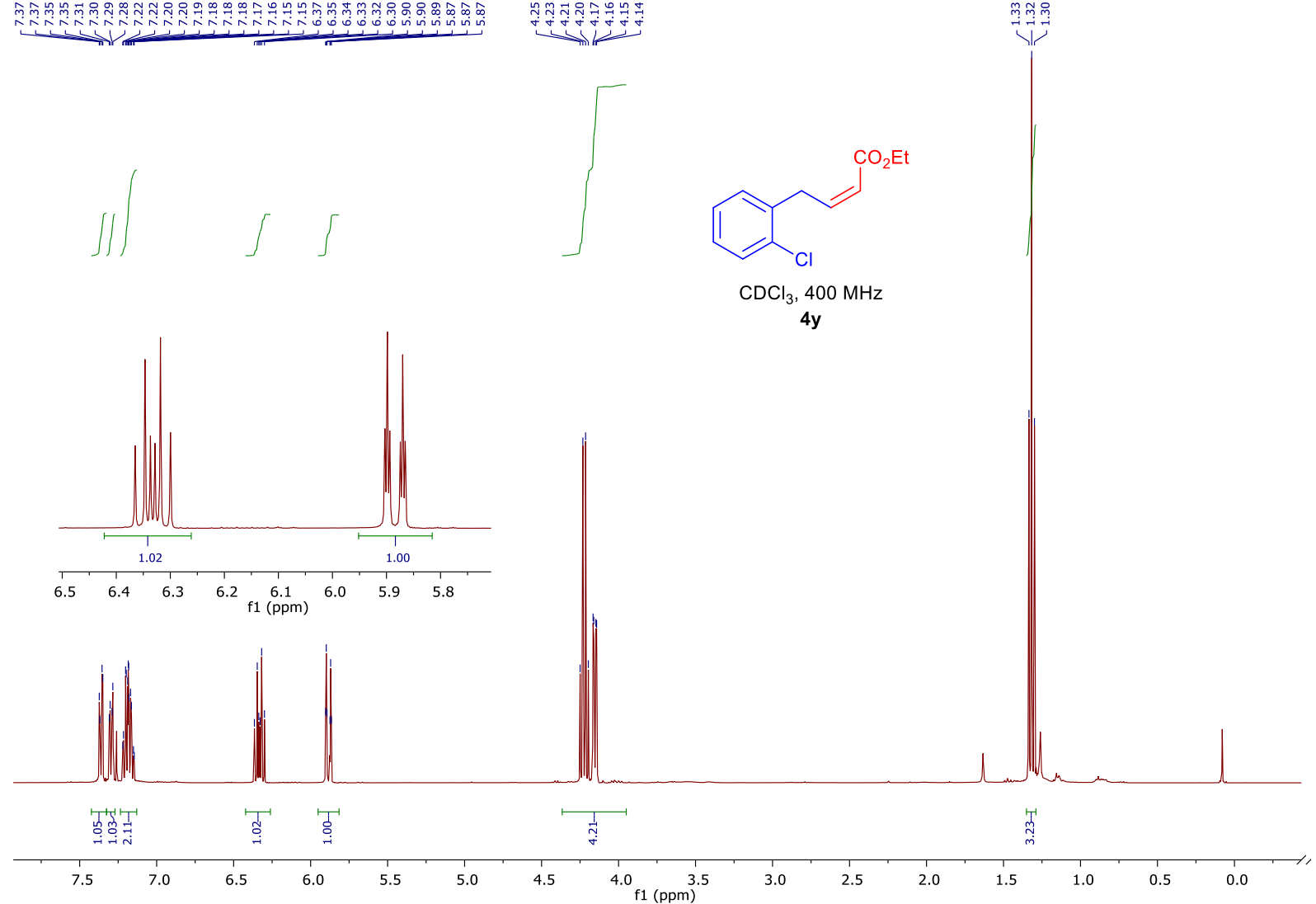




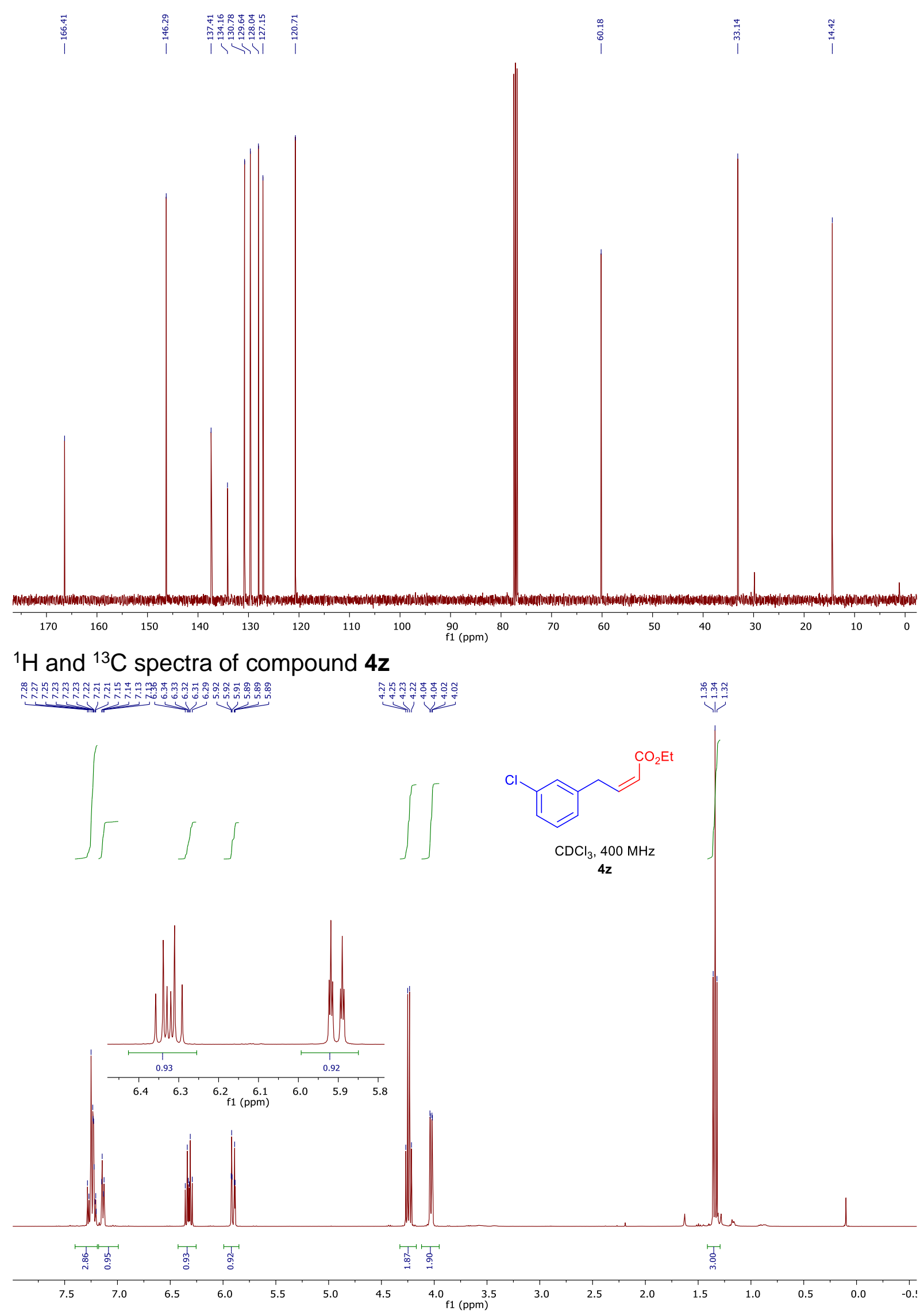




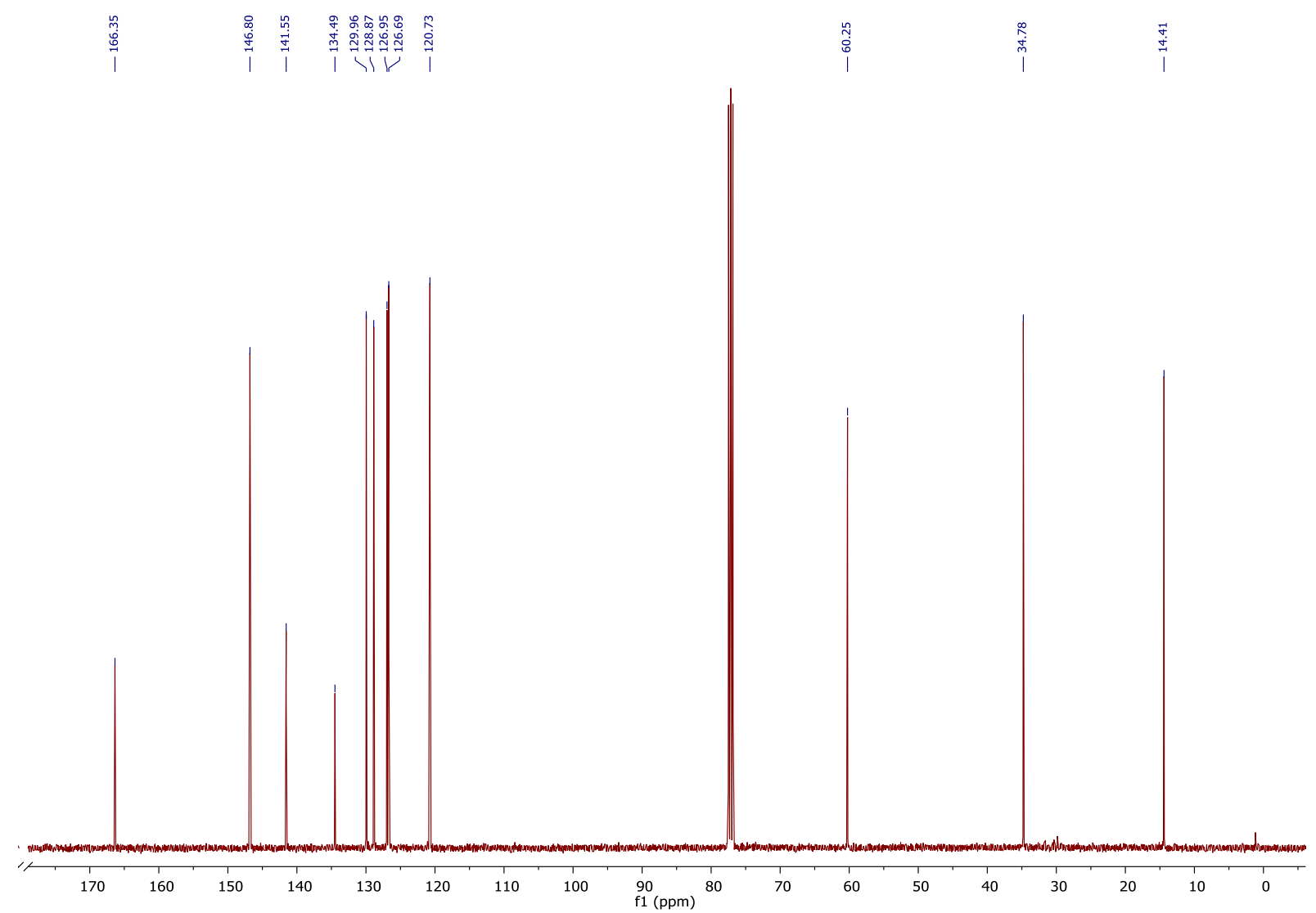

